\title{
INSTANTONS ON ALE SPACES, QUIVER VARIETIES, AND KAC-MOODY ALGEBRAS
}

\author{
HIRAKU NAKAJIMA \\ To Professor Shoshichi Kobayashi on his 60th birthday
}

1. Introduction. In this paper we shall introduce a new family of varieties, which we call quiver varieties, and study their geometric structures. They have close relation to the singularity theory and the representation theory of the KacMoody algebras.

Our original motivation was to study solutions of the anti-self-dual YangMills equations on a particular class of 4-dimensional noncompact complete manifolds, the so-called ALE spaces (or the ALE gravitational instantons), which were constructed by Kronheimer [Kr1]. In [KN] we gave a description of the framed moduli space of all solutions in terms of solutions of a system of quadratic equations (called the ADHM equations) for representations of a quiver on an affine, simply laced Dynkin graph. It is an analogue of the description, given by Atiyah, Drinfeld, Hitchin, and Manin [ADHM], of the moduli space for $\mathbb{R}^{4}$ (or $S^{4}$ ) in terms of solutions of a quadratic equation for certain finite-dimensional matrices.

Once we set aside their gauge-theoretic origin, there is no longer reason to restrict ourselves to affine Dynkin graphs. Definitions can be generalized to arbitrary finite graphs. We get what we call quiver varieties.

We study geometric structures of quiver varieties in this paper. In [Na1] it was noticed that the moduli space of anti-self-dual connections on ALE spaces has a hyper-Kähler structure, namely a Riemannian metric equipped with three endomorphisms $I, J, K$ of the tangent bundle which satisfy the relations of quaternion algebra and are covariant constant with respect to the Levi-Civita connection:

$$
I^{2}=J^{2}=K^{2}=-1, \quad I J=-J I=K, \quad \nabla I=\nabla J=\nabla K=0
$$

The same holds for general quiver varieties. In particular, quiver varieties have holomorphic symplectic forms. We study further properties of the quiver variety, such as a natural $\mathbb{C}^{*}$-action, symplectic geometry, topology, and so on. As ALE spaces closely related to simple singularities, quiver varieties have very special kinds of singularities that enjoy very nice properties.

Received 25 June 1993. Revision received 21 March 1994.

Author supported in part by Grant-in-Aid for Scientific Research (No. 05740041), Ministry of Education, Science, and Culture, Japan. 
Surprisingly, the ADHM equation appears in a very different context. In [L3] Lusztig used it to construct "canonical bases" of the - part $\mathbf{U}^{-}$of the quantized enveloping algebra $\mathbf{U}$ associated by Drinfeld and Jimbo to the graph. Motivated by his results, we give a geometric construction of irreducible highest-weight integrable representations of the Kac-Moody algebra associated to the graph (Theorem 10.14). The weight space of the representation space will be given as a vector space consisting of constructible functions on a Lagrangian subvariety of a quiver variety. The action of the Kac-Moody algebra, which maps a constructible function on a quiver variety to one on another quiver variety, is given by using "geometric Hecke operators". Thus the representation can be constructed if we treat several quiver varieties simultaneously. An advantage of our approach is that it gives a geometric construction of the action of the whole Kac-Moody algebra, not just the - part. We also observe (Theorem 10.16) that the middle cohomology group of the quiver variety is isomorphic to a weight space of an irreducible integrable highest-weight representation, when the underlying graph is of type ADE or affine.

Let us briefly summarize the context of this paper. In $\$ 2$ we give the definition of quiver varieties, a quick review of the construction of the ALE spaces by Kronheimer [Kr1], and the ADHM description of anti-self-dual connections on ALE spaces [KN]. The quiver varieties will be described as hyper-Kähler quotients (see Hitchin et al. [HKLR]) of representation spaces of a quiver on a graph, which are finite-dimensional quaternion vector spaces. The representations of the quiver that we use here are little bit different from those used in the literature. We put two vector spaces for each vertex (see (2.1)). We also choose a parameter $\zeta=\left(\zeta_{\mathbb{R}}, \zeta_{\mathbb{C}}\right)$ from $Z \oplus(Z \otimes \mathbb{C})$, where $Z$ is a finite-dimensional real vector space. So we denote the corresponding quiver variety by $\mathfrak{M}_{\zeta}$. The parameters which give nonsingular varieties form an open dense subset $(Z \oplus(Z \otimes \mathbb{C}))^{\circ}$ in $Z \oplus(Z \otimes \mathbb{C})$. Their underlying differentiable structures are independent of the parameter. If we move the parameter, the hyper-Kähler structure on the quiver variety changes correspondingly. Parameters in the complement $Z \oplus(Z \otimes \mathbb{C}) \backslash$ $(Z \oplus(Z \otimes \mathbb{C}))^{\circ}$ correspond to singular varieties. The variety has the most complicated singularities when $\zeta=0$. If we move the parameter in the $Z \otimes \mathbb{C}$-component, we get a deformation of the central fiber. The situation is quite similar to that of simple singularities.

The ADHM equation arises as the hyper-Kähler moment map equation. It decomposes into two components: the real ADHM equation and the complex ADHM equation. The real ADHM equation contains terms with the hermitian adjoint of matrices. In $\S 3$ we give a purely holomorphic description of the quiver variety, which does not deal with the hermitian inner product. Similar phenomena appeared when Donaldson studied anti-self-dual connections on $\mathbb{R}^{4}$ [Do]. The essential point is to introduce a subset $H_{\zeta}^{\mathrm{s}}$ of the affine variety given by the complex ADHM equation. The superscript "s" means the stability, the notion coming from the geometric invariant theory. The complexification $G_{\mathbf{v}}^{\mathbb{C}}$ of a compact Lie group $G_{\mathbf{v}}$ acts on $H_{\zeta}^{\mathrm{s}}$. Then the quiver variety $\mathfrak{M}_{\zeta}$ is isomorphic to the 
quotient space $H_{\zeta}^{\mathrm{s}} / G_{\mathbf{v}}^{\mathbb{C}}$. The result can be explained by using the language of connections: the moduli space of anti-self-dual connections is isomorphic to that of holomorphic vector bundles. This so-called Hitchin-Kobayashi correspondence was proved by Donaldson and Uhlenbeck-Yau for compact Kähler manifolds. For an ALE space, which is noncompact but has a simple asymptotic structure, it was proved recently by Bando [Ba].

It is important to study not only the single quiver variety but also the relationship between quiver varieties with different parameters. In $\$ 4$ we define a holomorphic map $\pi: \mathfrak{M}_{\left(\zeta_{\mathbb{R}}, \zeta_{\mathbb{C}}\right)} \rightarrow \mathfrak{M}_{\left(0, \zeta_{\mathbb{C}}\right)}$, which is a resolution of singularities. This can be considered as a generalization of a result in [Kr1] where the corresponding statement was shown for ALE spaces.

In $\$ 5$ we define a $\mathbb{C}^{*}$-action on the quiver variety. We then study the fixed point set $\mathfrak{F}$ of the action. We also study another variety $\mathfrak{L}=\pi^{-1}(0)$ where $\pi: \mathfrak{M}_{\left(\zeta_{\mathbb{R}}, 0\right)} \rightarrow$ $\mathfrak{M}_{0}$ is the map defined in $\S 4$. It is shown that $\mathfrak{L}$ is a Lagrangian variety with respect to the holomorphic symplectic form and homotopy equivalent to $\mathfrak{M}_{\left(\zeta_{\mathbb{R}}, 0\right)}$. (The $\mathbb{C}^{*}$-action gives a retraction map.) In particular, irreducible components of $\mathfrak{L}$ give a basis for the middle homology of $\mathfrak{M}_{\left(\zeta_{\mathbb{R}}, 0\right)}$. It gives us the "canonical basis" for the representation considered in $\$ 10$.

In $\S 6$ we define a stratification on the singular quiver variety $\mathfrak{M}_{\zeta}$. The dense stratum is the set of regular points of $\mathfrak{M}_{\zeta}$, and each lower stratum is isomorphic to the nonsingular locus of the quiver variety corresponding to the different data. We show that the map $\pi: \mathfrak{M}_{\left(\zeta_{\mathbb{R}}, \zeta_{\mathbb{C}}\right)} \rightarrow \mathfrak{M}_{\left(0, \zeta_{\mathbb{C}}\right)}$ is semismall in the sense of [BM, 1.1]. The stratification can be explained in the language of anti-self-dual connections. The nonsingular locus corresponds to the moduli space of anti-self-dual connections, and it has the natural completion given by Uhlenbeck's compactness theorem. When points fall into lower strata, the curvatures of the corresponding connections go to infinity at singular points of $X_{\zeta}$ and the limit is a connection on a different vector bundle.

In $\S 7$ we give concrete examples of $\mathfrak{M}_{\zeta}$. The cotangent bundle of a generalized flag manifold of type $A_{n}$ is obtained as a quiver variety on the graph of type $A_{m}$. (Note that $m$ may be different from $n$ in general.) It is the resolution of the singularities (generalized Springer's resolution) of the closures of a nilpotent orbit, which is isomorphic to $\mathfrak{M}_{0}$. The resolution map coincides with the one given in §3. Unfortunately, it seems very difficult to get such explicit descriptions of the quiver varieties in general. We do not know how to give generalized flag manifolds of other types in our theory.

In $\$ 8$ we give other examples. It is simply reformulation of a result of Kronheimer [ $\mathrm{Kr} 2]$. He constructed the intersection of a nilpotent orbit with a transversal slice to another nilpotent orbit as moduli spaces of SU(2)-equivariant anti-self-dual connections on $\mathbb{R}^{4}$. We can reformulate his result using a quiver when the Lie algebra is of type $A_{n}$ and show that $\mathfrak{M}_{0}$ is isomorphic to his variety in certain cases. Since $\mathfrak{M}_{\left(\zeta_{\mathbb{R}}, 0\right)}$ is a resolution of the singularities of $\mathfrak{M}_{0}$, it seems quite natural to conjecture that this coincides with the resolution constructed by Slodowy [S1]. We cannot verify this conjecture, but we show that their 
cohomology groups are isomorphic. Slodowy's resolutions contain subvarieties (called Spaltenstein's varieties in [BM]) as a deformation retract. BorhoMacPherson gave a formula for the Poincaré polynomials of Spaltenstein's varieties $[\mathrm{BM}]$.

In $\$ 9$ we give an application of our construction when the graph is of Dynkin type. As we already noticed, the quiver varieties are constructed as a family parametrized by $(Z \oplus(Z \otimes \mathbb{C}))^{\circ}$. The vector space $Z$ is identified with the real Cartan subalgebra and has an action of the Weyl group. The action lifts to the total space when the corresponding vector bundle satisfies $c_{1}(E)=0$. Then the monodromy representation gives us a Weyl group representation on the homology of the moduli space. This is very similar to the situations studied by Slodowy [S1]. (It is known that they coincide with the Springer representation. All irreducible representations of the Weyl group are realized by his construction.)

In $\$ 10$ we give a geometric construction of representations of the Kac-Moody algebra. For the construction of the action of its upper triangular part, we basically follow Lusztig's idea [L2], [L3], but our approach is different from his in two points. First, we have extra vector space $W_{k}$ for each vertex $k$. Second, we consider stable points $H_{\zeta}^{\mathrm{s}}$ instead of the whole variety. These lead us to construct representations instead of the - part $u^{-}$of the universal enveloping algebra.

A construction of representations of quantized enveloping algebra is also discussed in $\$ 11$. This part is not satisfactory yet, since we can only give a geometric construction of the action of $\mathbf{U}^{-}$. This is done by studying the microsupports of perverse sheaves, which was used to construct $\mathbf{U}^{-}$by Lusztig [L2], [L3].

By recalling results from $\S 8$, we get a mysterious connection between representations and Spaltenstein's varieties, at least for type $A$ (i.e., the dimension of the weight space is equal to the number of irreducible components of the Spaltenstein's variety). This may be confusing; the rank $n$ is not the same in general. Even if we study the quiver variety on the Dynkin graph of type $A_{n}$, it may correspond to Spaltenstein's variety of type $A_{m}$ with $m \neq n$. Ginzburg constructed representations of $\mathfrak{s l}_{n}$ on top-degree homology groups of Spaltenstein's varieties [Gi]. (See also Beilinson-Lusztig-MacPherson [BLM].) It is natural to conjecture that our representations are isomorphic to his ones via the results in $\S 8$. But the author has no idea for the proof at this moment.

Our study is reminiscent of Hitchin's self-duality equations [Hi]. It is a partial differential equation on Riemann surfaces, which is obtained by the dimensional reduction of the anti-self-duality equation on the 4-dimensional Euclidean space. Both moduli spaces, Hitchin's and ours, have hyper-Kähler structures and circle actions. Both theories have many examples of moduli spaces in common. Donaldson and Kronheimer described the cotangent bundle of the generalized flag manifold as the moduli space of $S^{1}$-equivariant solutions of the self-duality equation on the 2-disk, while it also appears in our theory (see \$7). So in the final section, we discuss analogies in both theories. 
We use the results in [Kr1], [KN] only in the last halves of $\S 2$ and $\S 3$ and also in $\S 9$. But those parts are written only for the explanation of the motivation. The main part of our paper is independent of [Kr1], [KN].

In [Na4], our results are explained in the gauge-theoretic terms, instead of the language of quivers.

Acknowledgement. I would like to thank Peter Kronheimer for a number of interesting discussions concerning the present work. I am also grateful to the referee, who introduced me to the work of King [Kin].

2. ALE spaces, the ADHM descriptions, and quiver varieties. The purpose of this section is to give the definition of quiver varieties and the ALE spaces, and to state the ADHM description on the ALE spaces. Our notation here will be a little different from the original one [KN]; we choose a particular complex structure $I$, breaking the natural symmetry between three complex structures $I, J, K$. We adapt the notation coming from the representation theory of quivers, veiling a finite subgroup $\Gamma$ of SU(2), which exists only in the case of affine Dynkin graphs.

Suppose a finite nonempty graph is given. Two different vertices may be joined by several edges, but we assume that no edge may join a vertex with itself. (This assumption can be dropped for some results in this paper. See [Na3].) An affine Dynkin graph of type $\widetilde{A}_{n}, \widetilde{D_{n}}, \widetilde{E_{6}}, \widetilde{E_{7}}$, or $\widetilde{E_{8}}$ satisfies the above assumption. Let $H$ be the set of pairs consisting of an edge together with an orientation of it. Let in $(h)$ (resp. out $(h)$ ) be the incoming (resp. outgoing) vertex of $h \in H$. For $h \in H$ we denote by $\bar{h}$ the same edge as $h$ with the reverse orientation. (Hence we have $\operatorname{in}(\bar{h})=\operatorname{out}(h), \operatorname{out}(\bar{h})=\operatorname{in}(h)$.) Choose a subset $\Omega \subset H$ such that $\bar{\Omega} \cup \Omega=H$, $\Omega \cap \bar{\Omega}=\varnothing$. Such a choice of the subset is called an orientation of the graph. The choice of the orientation is not essential. Our constructions are essentially independent of $\Omega$.

We assume that $\Omega$ has no cycles; i.e., we do not have $m \geqslant 2$ and $h_{1}, h_{2}, \ldots$, $h_{m} \in \Omega$ such that $\operatorname{in}\left(h_{i}\right)=\operatorname{out}\left(h_{i+1}\right)$ for $1 \leqslant i \leqslant m-1$ and $\operatorname{in}\left(h_{m}\right)=\operatorname{out}\left(h_{1}\right)$. This is possible if the graph has no edge joining a vertex with itself. We number the vertices and identify the set of vertices with $\{1,2, \ldots, n\}$, where $n$ is the number of vertices in the graph.

Suppose we are given pairs of hermitian vector spaces $V_{k}, W_{k}$ for each vertex $k$. Their dimensions are the vectors

$$
\mathbf{v}={ }^{t}\left(\operatorname{dim}_{\mathbb{C}} V_{1}, \ldots, \operatorname{dim}_{\mathbb{C}} V_{n}\right), \quad \mathbf{w}={ }^{t}\left(\operatorname{dim}_{\mathbb{C}} W_{1}, \ldots, \operatorname{dim}_{\mathbb{C}} W_{n}\right) \in\left(\mathbb{Z}_{\geqslant 0}\right)^{n}
$$

Let us define a complex vector space $\mathbf{M}$ by

$$
\mathbf{M} \stackrel{\text { def. }}{=}\left(\bigoplus_{h \in H} \operatorname{Hom}\left(V_{\text {out }(h)}, V_{\text {in }(h)}\right)\right) \oplus\left(\bigoplus_{k=1}^{n} \operatorname{Hom}\left(W_{k}, V_{k}\right) \oplus \operatorname{Hom}\left(V_{k}, W_{k}\right)\right)
$$

When we want to emphasize the dimensions, we use the notation $\mathbf{M}(\mathbf{v}, \mathbf{w})$. For an element of $\mathbf{M}$ we denote its components by $B_{h}, i_{k}, j_{k}$. We write $B, i, j$ for the 
collection $\left(B_{h}\right)_{h \in H}$, etc. The dimension of $\mathbf{M}$ is given by

$$
\operatorname{dim}_{\mathbb{R}} \mathbf{M}=2^{t} \mathbf{v A v}+4^{t} \mathbf{v w},
$$

where $\mathbf{A}=\left(a_{k l}\right)$ is the adjacency matrix of the graph, i.e.,

$$
a_{k l}=a_{l k}=\text { the number of edges joining } k \text { and } l .
$$

We define a symplectic form $\omega_{\mathbb{C}}$ on $\mathbf{M}$ by

$$
\omega_{\mathbb{C}}\left((B, i, j),\left(B^{\prime}, i^{\prime}, j^{\prime}\right)\right) \stackrel{\text { def. }}{=} \sum_{h \in H} \operatorname{tr}\left(\varepsilon(h) B_{h} B_{\bar{h}}^{\prime}\right)+\sum_{k=1}^{n} \operatorname{tr}\left(i_{k} j_{k}^{\prime}-i_{k}^{\prime} j_{k}\right),
$$

where $\varepsilon(h)=1$ if $h \in \Omega, \varepsilon(h)=-1$ if $h \in \overline{\mathbf{\Omega}}$. The symplectic vector space $\mathbf{M}$ decomposes into the sum $\mathbf{M}=\mathbf{M}_{\boldsymbol{\Omega}} \oplus \mathbf{M}_{\overline{\mathbf{\Omega}}}$ of Lagrangian subspaces:

$$
\begin{gathered}
\mathbf{M}_{\Omega} \stackrel{\text { def. }}{=}\left(\bigoplus_{h \in \Omega} \operatorname{Hom}\left(V_{\text {out }(h)}, V_{\text {in }(h)}\right)\right) \oplus\left(\bigoplus_{k=1}^{n} \operatorname{Hom}\left(W_{k}, V_{k}\right)\right) \\
\mathbf{M}_{\bar{\Omega}} \stackrel{\text { def. }}{=}\left(\bigoplus_{h \in \bar{\Omega}} \operatorname{Hom}\left(V_{\text {out }(h)}, V_{\text {in }(h)}\right)\right) \oplus\left(\bigoplus_{k=1}^{n} \operatorname{Hom}\left(V_{k}, W_{k}\right)\right) .
\end{gathered}
$$

We can consider $\mathbf{M}_{\bar{\Omega}}$ as a dual space of $\mathbf{M}_{\Omega}$ via $\omega_{\mathbb{C}}$, and $\mathbf{M}$ as the cotangent bundle of $\mathbf{M}_{\mathbf{\Omega}}$.

Since $V$ and $W$ are hermitian vector spaces, $\operatorname{Hom}(V, W)$ has a hermitian inner product defined by $(f, g)=\operatorname{tr}\left(f g^{\dagger}\right)$, where $(\bullet)^{\dagger}$ is the hermitian adjoint. We introduce a natural hermitian inner product on $\mathbf{M}$ induced from ones on $V_{k}$ and $W_{k}$ in this way. We introduce a quaternion module structure on $\mathbf{M}$ by the original complex structure $I$ together with a new complex structure $J$ given by the formula $J\left(m, m^{\prime}\right)=\left(-m^{\prime \dagger}, m^{\dagger}\right)$ for $\left(m, m^{\prime}\right) \in \mathbf{M}_{\Omega} \oplus \mathbf{M}_{\bar{\Omega}}$.

The group $G_{\mathbf{v}}=\prod \mathrm{U}\left(V_{k}\right)$ acts on $\mathbf{M}$ by

$$
\left(B_{h}, i_{k}, j_{k}\right) \mapsto\left(g_{\mathrm{in}(h)} B_{h} g_{\mathrm{out}(h)}^{-1}, g_{k} i_{k}, j_{k} g_{k}^{-1}\right),
$$

preserving the hermitian inner product and the $\mathbb{H}$-module structure. Let $\mu$ be the corresponding hyper-Kähler moment map (see Hitchin et al. [HKLR]) vanishing at the origin. Denote by $\mu_{\mathbb{R}}, \mu_{\mathbb{C}}$ its real and complex components. Explicit forms are as follows:

$$
\mu_{\mathbb{R}}(B, i, j)=\frac{i}{2}\left(\sum_{h \in H: k=\mathrm{in}(h)} B_{h} B_{h}^{\dagger}-B_{\bar{h}}^{\dagger} B_{\bar{h}}+i_{k} i_{k}^{\dagger}-j_{k}^{\dagger} j_{k}\right)_{k} \in \underset{k}{\bigoplus} \mathfrak{u}\left(V_{k}\right)=\mathfrak{g}_{\mathbf{v}},
$$

$$
\mu_{\mathbb{C}}(B, i, j)=\left(\sum_{h \in H: k=\mathrm{in}(h)} \varepsilon(h) B_{h} B_{\bar{h}}+i_{k} j_{k}\right)_{k} \in \bigoplus_{k} \mathfrak{g l}\left(V_{k}\right)=\mathfrak{g}_{\mathbf{v}} \otimes \mathbb{C},
$$

where $g_{v}$ is the Lie algebra of $G_{v}$, and it is identified with its dual space $\mathfrak{g}_{v}^{*}$ via the 
above hermitian inner product. Note that our moment map differs from Kirwan's by a sign.

Let $Z_{\mathbf{v}} \subset \mathfrak{g}_{\mathbf{v}}$ denote the center. Choose an element $\zeta=\left(\zeta_{\mathbb{R}}, \zeta_{\mathbb{C}}\right) \in Z_{\mathbf{v}} \oplus\left(Z_{\mathbf{v}} \otimes \mathbb{C}\right)$, and define a hyper-Kähler quotient $\mathfrak{M}_{\zeta}$ of $\mathbf{M}$ by $G_{\mathbf{v}}$ as follows:

$$
\mathfrak{M}_{\zeta}=\mathfrak{M}_{\zeta}(\mathbf{v}, \mathbf{w}) \stackrel{\text { def. }}{=}\{(B, i, j) \in \mathbf{M} \mid \mu(B, i, j)=-\zeta\} / G_{\mathbf{v}} .
$$

This is the quiver variety, which is the focus of our study.

Notation. We denote by $[(B, i, j)]$ the $G_{\mathbf{v}}$-orbit of $(B, i, j)$ considered as a point in $\mathfrak{M}_{\zeta}$.

The equations for $\tilde{A_{3}}$ are visualized in [KN, Introduction]. We call $\mu_{\mathbb{R}}(B, i, j)=$ $-\zeta_{\mathbb{R}}$ (resp. $\left.\mu_{\mathbb{C}}(B, i, j)=-\zeta_{\mathbb{C}}\right)$ the real (resp. complex) ADHM equation. We remark that $\mathfrak{M}_{\zeta}$ may be an empty set.

In general, $\mathfrak{M}_{\zeta}$ has singularities. We take a subset

$$
\mathfrak{M}_{\zeta}^{\text {reg def. }} \stackrel{\text { def }}{=}\left\{(B, i, j) \in \mu^{-1}(-\zeta) \mid \text { the stabilizer of }(B, i, j) \text { in } G_{\mathbf{v}} \text { is trivial }\right\} / G_{\mathbf{v}} .
$$

By the general theory of hyper-Kähler quotients [HKLR], this is a nonsingular hyper-Kähler manifold (provided it is nonempty), and its dimension is given by the formula

$$
\operatorname{dim}_{\mathbb{R}} \mathfrak{M}_{\zeta}^{\mathrm{reg}}=\operatorname{dim}_{\mathbb{R}} M-4 \operatorname{dim}_{\mathbb{R}} G_{\mathbf{v}}=2^{t} \mathbf{v}(2 \mathbf{w}-\mathbf{C v})
$$

(cf. (2.2)). Here $\mathbf{C}=2 \mathbf{I}-\mathbf{A}$ is the generalized Cartan matrix. The holomorphic symplectic form on $\mathfrak{M}_{\zeta}^{\text {reg }}$ will be denoted by $\omega_{\mathbb{C}}$.

The center $Z_{\mathbf{v}}$ of $\mathfrak{g}_{\mathbf{v}}$ is the product of the set of scalar matrices on $V_{k}$. Taking scalars, we can consider $Z_{v}$ as a subspace of $\mathbb{R}^{n}$. (Since some $V_{k}$ may be $0, Z_{\mathbf{v}}$ may not be the whole $\mathbb{R}^{n}$.) In $\S 10$, we shall associate a Kac-Moody algebra to the given graph. Then $\mathbb{R}^{n}$ will be identified with the real Cartan subalgebra.

Let

$$
\begin{aligned}
R_{+} & \stackrel{\text { def. }}{=}\left\{\theta=\left.\left(\theta_{k}\right) \in \mathbb{Z}_{\geqslant 0}^{n}\right|^{t} \theta \mathbf{C} \theta \leqslant 2\right\} \backslash\{0\} \\
R_{+}(\mathbf{v}) & \stackrel{\text { def. }}{=}\left\{\theta \in R_{+} \mid \theta_{k} \leqslant \operatorname{dim}_{\mathbb{C}} V_{k} \text { for all } k\right\} \\
D_{\theta} & \stackrel{\text { def. }}{=}\left\{x=\left(x_{k}\right) \in \mathbb{R}^{n} \mid \sum_{k} x_{k} \theta_{k}=0\right\} \quad \text { for } \theta \in R_{+} .
\end{aligned}
$$

When the graph is of Dynkin type, $R_{+}$is the set of positive roots, and $D_{\theta}$ is the wall defined by the root $\theta$. In general, $R_{+}$may be an infinite set, but $R_{+}(\mathbf{v})$ is always finite.

TheOREM 2.8. Suppose

$$
\zeta \in \mathbb{R}^{3} \otimes \mathbb{R}^{n} \backslash \bigcup_{\theta \in R_{+}(\mathbf{v})} \mathbb{R}^{3} \otimes D_{\theta}
$$


Then the regular locus $\mathfrak{M}_{\zeta}^{\text {reg }}$ coincides with $\mathfrak{M}_{\zeta}$. Thus $\mathfrak{M}_{\zeta}$ is nonsingular. Moreover, the hyper-Kähler metric is complete.

Definition 2.10. We say $\zeta$ is generic if it satisfies the condition (2.9).

Proof of (2.8). The proof is essentially the same as [Kr1, 2.8]. Suppose that $(B, i, j) \in \mu^{-1}(-\zeta)$ is fixed by nontrivial element $g=\left(g_{k}\right) \in G_{\mathbf{v}}$. Let

$$
V_{k}=\bigoplus_{\lambda} V_{k}(\lambda)
$$

be the eigenspace decomposition of $V_{k}$ with respect to $g_{k}$. Then

$$
B_{h}\left(V_{\text {out }(h)}(\lambda)\right) \subset V_{\text {in }(h)}(\lambda), \quad i_{k}\left(W_{k}\right) \subset V_{k}(1), \quad j_{k}\left(V_{k}(\lambda)\right)=0 \quad \text { unless } \lambda=1 .
$$

Choose $\lambda \neq 1$ and set $\mathbf{v}^{\prime}=\left(\operatorname{dim}_{\mathbb{C}} V_{k}(\lambda)\right)_{k}$. The above implies that $\left(B_{h}\right)_{h \in H}$ defines an element in $\mathfrak{M}\left(\mathbf{v}^{\prime}, 0\right)$. If $G_{\mathbf{v}^{\prime}} / \mathrm{U}(1)$ does not act freely on the $\left(B_{h}\right)$-orbit, we can further decompose $V_{k}^{\prime}$. Finally we may assume that this condition is met. Hence $\mu^{-1}(-\zeta) /\left(G_{\mathbf{v}^{\prime}} / \mathrm{U}(1)\right)$ is a manifold of at least one point. So the dimension formula implies

$$
\operatorname{dim}_{\mathbb{C}} \mu^{-1}(-\zeta) /\left(G_{\mathbf{v}^{\prime}} / \mathbf{U}(1)\right)=2-{ }^{t} \mathbf{v}^{\prime} \mathbf{C v}^{\prime} \geqslant 0
$$

Thus we have $\mathbf{v}^{\prime} \in R_{+}(\mathbf{v})$.

Let $\pi_{k}$ be the orthogonal projection to $V_{k}^{\prime}$ in $V_{k}$. Then $\left(\exp \left(\sqrt{-1} \pi_{k}\right)\right)_{k} \in G$ fixes $(B, i, j)$. Hence

$$
0=\left\langle\mu(B, i, j),\left(\sqrt{-1} \pi_{k}\right)_{k}\right\rangle=-\left\langle\zeta,\left(\sqrt{-1} \pi_{k}\right)_{k}\right\rangle=\sum_{k} \zeta_{k} \operatorname{dim}_{\mathbb{C}} V_{k}^{\prime}
$$

where $\zeta=\left(\zeta_{k}\right)_{k} \in(\mathbb{R} \oplus \mathbb{C})^{n}$. Hence $\zeta \in \mathbb{R}^{3} \otimes D_{\mathbf{v}^{\prime}}$.

The last two statements follow from the general theory of hyper-Kähler quotients.

The rest of this section is devoted to giving the definition of ALE spaces and to explaining the ADHM description. They provide the motivation for our definition of quiver varieties.

The ALE spaces can also be described as $\mathfrak{M}_{\zeta}(\mathbf{v}, \mathbf{w})$ for specific data $\mathbf{v}, \mathbf{w}$ on the affine Dynkin graph. In this case, it is customary to number the vertex so that 0 is the vertex corresponding to the negative of the highest root of the corresponding

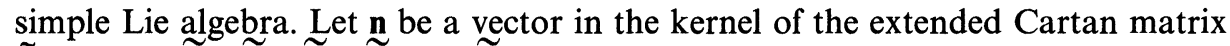
$\widetilde{\mathbf{C}}$ of type $\widetilde{A}_{n}, \widetilde{D_{n}}, \widetilde{E_{6}}, \widetilde{E_{7}}$, or $\widetilde{E}_{8}$. The kernel is 1-dimensional, so $\mathbf{n}$ is uniquely determined if we normalize so that the 0 th component $n_{0}$ is equal to 1 . Other components are coefficients of the highest root. We consider the case that the dimensions are given by $\mathbf{v}=\mathbf{n}, \mathbf{w}=0$. Then the group $U(1)$ of scalars acts trivially, so we consider the action of the quotient group $G_{\mathbf{n}}^{\prime}=G_{\mathbf{n}} / \mathrm{U}(1)$. Choose $\zeta=$ 
$\left(\zeta_{\mathbb{R}}, \zeta_{\mathbb{C}}\right) \in Z \oplus(Z \otimes \mathbb{C})$, where $Z \subset \mathrm{g}_{\mathbf{n}}$ is the trace-free part of the center. It is identified with the center of the Lie algebra of $G_{\mathbf{n}}^{\prime}$. Now define

$$
X_{\zeta} \stackrel{\text { def. }}{=}\{B \in \mathbf{M}(\mathbf{n}, 0) \mid \mu(B)=\zeta\} / G_{\mathbf{n}}^{\prime} .
$$

Be careful with the sign on the parameter $\zeta$; here we use the equation $\mu(B)=\zeta$ instead of $\mu(B)=-\zeta$. This is because our quiver variety $\mathfrak{M}_{\zeta}$ will be described as a moduli space of instantons on $X_{\zeta}$, not on $X_{-\zeta}$ (see Proposition 2.15 below). Then the main result of $[\mathrm{Kr} 1]$ can be summarized as follows.

Proposition 2.12. Suppose that

$$
\zeta \in \mathbb{R}^{3} \otimes Z \backslash \bigcup_{\theta \in R_{+} \backslash\{\mathbf{n}\}} \mathbb{R}^{3} \otimes D_{\theta} .
$$

Then the action of $G_{\mathbf{n}}^{\prime}$ on $\mu^{-1}(\zeta)$ is free, and the quotient $X_{\zeta}$ is a smooth 4-dimensional hyper-Kähler manifold; it is diffeomorphic to the minimal resolution of $\mathbb{C}^{2} / \Gamma$, and the metric is ALE; here $\Gamma$ is the finite subgroup of $\mathrm{SU}(2)$ associated to the Dynkin graph.

The ALE condition means there exists a coordinate system at infinity $\mathfrak{X}$ : $X_{\zeta} \backslash K \rightarrow\left(\mathbb{C}^{2} \backslash \overline{B_{R}}\right) / \Gamma$ for some compact set $K$, and the metric approximates the Euclidean metric on $\mathbb{C}^{2} / \Gamma$. Note that $\zeta$ is in $D_{\mathbf{n}}$, since it is trace-free.

The ALE spaces are fundamental among the spaces $\mathfrak{M}_{\zeta}(\mathbf{v}, \mathbf{w})$; they are 4-dimensional, i.e., they have the lowest possible positive dimension. They are fundamental by another reason. The other spaces are obtained as moduli spaces of antiself-dual connections on ALE spaces, as we explain below.

The construction of ALE spaces gives a natural principal $G_{\mathrm{n}}^{\prime}$-bundle $\mu^{-1}(\zeta) \rightarrow$ $X_{\zeta}$. This bundle has a natural connection [GN]; the horizontal subspace is the orthogonal complement to the fiber direction. Identifying $G_{n}^{\prime}$ with $\prod_{k \neq 0} \mathrm{U}\left(n_{k}\right)$ $\left(\mathbf{n}={ }^{t}\left(n_{0}, \ldots, n_{n}\right)\right)$, we consider an associated vector bundle

$$
\mathscr{R}_{l}=\mu^{-1}(\zeta) \times_{G_{\mathrm{n}}^{\prime}} R_{l} \quad(l=1, \ldots, n),
$$

where $R_{l}=\mathbb{C}^{n_{l}}$, on which $\mathrm{U}\left(n_{k}\right)$ acts trivially unless $k=l$, and $\mathrm{U}\left(n_{l}\right)$ acts naturally. Let $\mathscr{R}_{0}$ be the trivial bundle. From the definition of $X_{\zeta}$ there is also a tautological vector bundle endomorphism

$$
\xi=\left(\xi_{h}\right) \in \bigoplus_{h \in H} \operatorname{Hom}\left(\mathscr{R}_{\text {out }(h)}, \mathscr{R}_{\text {in }(h)}\right) .
$$

The parameter $\zeta \in Z \oplus(Z \otimes \mathbb{C})$ determines an element, for which we use the same symbol $\zeta$, in $Z_{\mathbf{v}} \oplus\left(Z_{\mathbf{v}} \otimes \mathbb{C}\right)$ as follows. In the decomposition $\mathfrak{g}_{\mathbf{n}}=\bigoplus_{k} \mathfrak{u}\left(n_{k}\right)$, the $k$ th component of $\zeta$ is a scalar matrix of size $n_{k}$. Multiplying the identity matrix of size $\operatorname{dim} V_{k}$ by the same scalar, we consider $\zeta$ as an element of $Z_{\mathbf{v}} \oplus$ $\left(Z_{\mathbf{v}} \otimes \mathbb{C}\right)$. 
Take $(B, i, j) \in \mu^{-1}(-\zeta)$ and consider the vector bundle homomorphisms

$$
\bigoplus_{k} V_{k} \otimes \mathscr{R}_{k} \stackrel{\sigma}{\rightarrow}\left(\bigoplus_{h \in H} V_{\text {out }(h)} \otimes \mathscr{R}_{\mathrm{in}(h)}\right) \oplus\left(\bigoplus_{k} W_{k} \otimes \mathscr{R}_{k}\right) \stackrel{\tau}{\rightarrow} \bigoplus_{k} V_{k} \otimes \mathscr{R}_{k}
$$

where

$$
\begin{aligned}
& \sigma=\left(B_{\bar{h}} \otimes 1_{\mathscr{R}_{\mathrm{in}(h)}}+\varepsilon(h) 1_{V_{\text {out }(h)}} \otimes \xi_{h}\right) \oplus j_{k} \otimes 1_{\mathscr{R}_{k}}, \\
& \tau=\left(\varepsilon(h) B_{h} \otimes 1_{\mathscr{R}_{\mathrm{in}(h)}}-1_{V_{\text {out }(h)}} \otimes \xi_{\bar{h}}, i_{k} \otimes 1_{V_{k}}\right) .
\end{aligned}
$$

Here 1. is the identity map. Then the complex $\operatorname{ADHM}$ equation $\mu_{\mathbb{C}}(B, i, j)=-\zeta_{\mathbb{C}}$ implies that $\tau \sigma=0$, and so (2.14) is a complex. The condition of the trivial stabilizer is equivalent to saying that $\sigma$ is injective and $\tau$ is surjective $[\mathrm{KN}, 9.2]$. Then [KN, 4.1] says the induced connection $A$ on the bundle

$$
E=\operatorname{Coker}\left(\sigma, \tau^{\dagger}\right) \subset\left(\bigoplus_{h \in H} V_{\text {out }(h)} \otimes \mathscr{R}_{\text {in }(h)}\right) \oplus\left(\bigoplus_{k} W_{k} \otimes \mathscr{R}_{k}\right)
$$

is anti-self-dual (the real ADHM equation is used here) and has the asymptotic behavior

$$
A-A_{0}=O\left(r^{-3}\right), \quad \nabla_{A_{0}}\left(A-A_{0}\right)=O\left(r^{-4}\right), \ldots,
$$

where $A_{0}$ is a flat connection defined on $E \mid\left(X_{\zeta} \backslash K\right), \nabla_{A_{0}}$ denotes the covariant derivatives associated with $A_{0}$, and $r$ is the absolute value $|\mathfrak{X}|$ of the coordinate system at infinity. Conversely, any such connection is obtained by this ADHM description [KN, 5.6].

Denote by $\mathscr{A}^{\text {asd }}$ the set of anti-self-dual connections $A$ satisfying the above asymptotic behavior. It has an action of the group $\mathscr{G}_{0}$ of gauge transformations $\gamma$ satisfying

$$
\gamma-1_{E}=O\left(r^{-2}\right), \quad \nabla_{A_{0}}\left(\gamma-1_{E}\right)=O\left(r^{-3}\right), \ldots,
$$

where $1_{E}$ is the identity transformation of $E$. We call the quotient space $\mathfrak{M H}_{\zeta}(E)=$ $\mathscr{A}^{\text {asd }} / \mathscr{G}_{0}$ the framed moduli space of anti-self-dual connections. In fact, our space $\mathfrak{M}_{\zeta}(E)$ is identified with the framed moduli space of instantons on the 1-point compactification $\bar{X}_{\zeta}=X_{\zeta} \cup\{\infty\}$ (framed at $\infty$ ). The main result of $[\mathrm{KN}]$ can be summarized as follows.

Proposition 2.15. When $\zeta$ satisfies (2.13), the ADHM description gives a oneto-one correspondence between $\mathfrak{M}_{\zeta}(E)$ and $\mathfrak{M}_{\zeta}^{\mathrm{reg}}(\mathbf{v}, \mathbf{w})$ for some $\mathbf{v}, \mathbf{w}$. (The data $\mathbf{v}, \mathbf{w}$ are determined from the topological data of $E$ and $A_{0}$.) 
Remark 2.16. The parameter $\zeta \in Z$, even if it satisfies (2.13), is not generic in the sense of $(2.10)$ in general. ( $\zeta$ is always in $D_{\mathbf{n}}$.) However, when $V_{0}=0$ (this means the data are defined on the Dynkin graph with the extra vertex 0 removed), the condition (2.13) is precisely the genericity condition. So when we study the quiver variety $\mathfrak{M}_{\zeta}$ on the Dynkin graph with generic parameter $\zeta$ in later sections, it will be the study of the framed moduli space of anti-self-dual connections on the ALE space $X_{\zeta}$.

3. A holomorphic description of $\mathfrak{M}_{\zeta}$. An affine variety (with respect to $I$ ) given by the equation $\mu_{\mathbb{C}}(B, i, j)=-\zeta_{\mathbb{C}}$ is invariant under the action of the complexification $G_{\mathrm{v}}^{\mathbb{C}}=\prod \mathrm{GL}\left(V_{k}\right)$ of $G_{\mathrm{v}}=\prod \mathrm{U}\left(V_{k}\right)$. When the real component $\zeta_{\mathbb{R}}$ of the parameter $\zeta$ is zero (i.e., $\zeta=\left(0, \zeta_{\mathbb{C}}\right)$ ), we have an isomorphism between the Kähler quotient and the affine algebro-geometric quotient by results of Kirwan and Ness [Ki], [Ne] (see also [Nee], [Sc]).

\section{THEOREM 3.1. There is a homeomorphism}

$$
\mathfrak{M}_{\left(0, \zeta_{\mathbb{C}}\right)}=\left(\mu_{\mathbb{R}}^{-1}(0) \cap \mu_{\mathbb{C}}^{-1}\left(-\zeta_{\mathbb{C}}\right)\right) / G_{\mathbf{v}} \stackrel{\cong}{\rightrightarrows} \mu_{\mathbb{C}}^{-1}\left(-\zeta_{\mathbb{C}}\right) / / G_{\mathbf{v}}^{\mathbb{C}},
$$

where // means the affine algebro-geometric quotient.

In fact, the authors have dealt with the more involved case of projective varieties in [Ki], [Ne]. Our situation is much more simple, since every critical point of $\left\|\mu_{\mathbb{R}}\right\|^{2}$ is in $\mu_{\mathbb{R}}^{-1}(0)$. The noncompactness causes no trouble since the natural inclusion $\mathfrak{M}_{\left(0, \zeta_{\mathbb{C}}\right)} \rightarrow \mu_{\mathbb{C}}^{-1}\left(-\zeta_{\mathbb{C}}\right) / / G_{\mathbb{v}}^{\mathbb{C}}$ is proper (see the proof of Theorem 4.1 ).

Next consider the case without $\zeta_{\mathbb{R}}=0$. Let us define the set of "stable" points by

$H_{\zeta}^{\text {s def. }}=\left\{m \in \mu_{\mathbb{C}}^{-1}\left(-\zeta_{\mathbb{C}}\right) \mid\right.$ the $G_{\mathbf{v}}^{\mathbb{C}}$-orbit through $m$ intersects the level set $\left.\mu_{\mathbb{R}}^{-1}\left(-\zeta_{\mathbb{R}}\right)\right\}$.

Also by the results of [Ki], [Ne], we have the following.

THEOREM 3.2. Suppose that $\zeta=\left(\zeta_{\mathbb{R}}, \zeta_{\mathbb{C}}\right)$ is generic. Then

(1) we have a homeomorphism

$$
\mathfrak{M}_{\zeta} \cong H_{\zeta}^{\mathrm{s}} / G_{\mathbf{v}}^{\mathbb{C}},
$$

(2) $\mu_{\mathbb{C}}^{-1}\left(-\zeta_{\mathbb{C}}\right) \backslash H_{\zeta}^{\mathrm{s}}$ is a complex subvariety of $\mu_{\mathbb{C}}^{-1}\left(-\zeta_{\mathbb{C}}\right)$.

Remarks 3.3. (1) For general complex projective manifolds with the assumption that the stabilizer of every point in $\mu_{\mathbb{R}}^{-1}(0)$ is finite, it is proved [Ki], [Ne] that $G^{\mathbb{C}} \mu_{\mathbb{R}}^{-1}(0)$ is the set of stable points in the sense of the geometric invariant theory.

(2) When the parameter $\zeta$ is not generic, statement (2) in the theorem becomes a little more complicated. Since we do not need the general statement, we leave it in the above form.

(3) When $\left(0, \zeta_{\mathbb{C}}\right)$ is generic, we have $H_{\left(\zeta_{\mathbb{R}}, \zeta_{\mathbb{C}}\right)}^{\mathrm{s}}=\mu_{\mathbb{C}}^{-1}\left(-\zeta_{\mathbb{C}}\right)$ for any $\zeta_{\mathbb{R}}$, i.e., all points are stable. Hence we have an isomorphism between complex manifolds 
$\mathfrak{M}_{\left(\zeta_{\mathbb{R}}, \zeta_{\mathbb{C}}\right)} \cong \mu_{\mathbb{C}}\left(-\zeta_{\mathbb{C}}\right) / G_{\mathfrak{v}}^{\mathbb{C}}$. (This is proved by the same argument as in $[\mathrm{Kr} 1,3.10]$.) In this case, the complex structure is independent of $\zeta_{\mathbb{R}}$, and $\zeta_{\mathbb{R}}$ parametrizes the Kähler metric.

Our definition of $H_{\zeta}^{\text {s }}$ uses the Kähler metric. But there is an alternative definition which makes sense for any base field. This kind of problem is discussed in a recent preprint by King [Kin]. We here give a differential geometric approach when the parameter $\zeta_{\mathbb{R}}=\left((i / 2) \zeta_{\mathbb{R}}^{(1)}, \ldots,(i / 2) \zeta_{\mathbb{R}}^{(n)}\right)$ satisfies the following condition:

$$
\zeta_{\mathbb{R}}^{(k)}>0 \quad \text { for } k=1,2, \ldots, n \text {. }
$$

Note that $\left(\zeta_{\mathbb{R}}, \zeta_{\mathbb{C}}\right)$ is generic under this assumption.

Proposition 3.5. Assume $\zeta=\left(\zeta_{\mathbb{R}}, \zeta_{\mathbb{C}}\right)$ satisfies condition (3.4). For $(B, i, j) \in$ $\mu_{\mathbb{C}}^{-1}\left(-\zeta_{\mathbb{C}}\right)$, the following two conditions are equivalent:

(1) If a collection $S=\left(S_{k}\right)_{k=1, \ldots, n}$ of subspaces of $V_{k}$ satisfies

$$
B_{h}\left(S_{\mathrm{out}(h)}\right) \subset S_{\mathrm{in}(h)}, \quad j_{k}\left(S_{k}\right)=0 \quad \text { for all } h \in H, k,
$$

then $S_{k}=0$ for all $k$;

(2) $(B, i, j) \in H_{\zeta}^{\mathrm{s}}$.

Proof. The proof has many similarities with the proof for the HitchinKobayashi correspondence. (Compare with [DK, §6].)

First we prove $(2) \Rightarrow(1)$. The set $H_{\zeta}^{\text {s }}$ consists of, by definition, points $m$ whose $G_{v}^{\mathbb{C}}$-orbit intersects with $\mu_{\mathbb{R}}^{-1}\left(-\zeta_{\mathbb{R}}\right)$. Since $(1)$ is a $G_{v}^{\mathbb{C}}$-invariant condition, we may assume that $(B, i, j)$ satisfies the real $\mathrm{ADHM}$ equation $\mu_{\mathbb{R}}(B, i, j)=-\zeta_{\mathbb{R}}$.

Suppose that a collection $S=\left(S_{k}\right)_{k}$ of subspaces as in (1) is given. Let $\pi_{k}$ be the orthogonal projection to $S_{k}$ in $V_{k}$. Let us write $B_{h}^{S}$ for the restriction of $B_{h}$ to $S_{\text {out }(h)}$. Considering it as a homomorphism from $S_{\text {out }(h)}$ to $S_{\text {in }(h)}$, we denote its adjoint by $\left(B_{\mathrm{h}}^{S}\right)^{+}: S_{\mathrm{in}(h)} \rightarrow S_{\text {out }(h)}$. It is given by

$$
\left(B_{h}^{S}\right)^{\dagger}=\left.\pi_{\text {out }(h)} B_{h}^{\dagger}\right|_{S_{\mathrm{in}(h)}},
$$

where $\left.\right|_{S_{\mathrm{in}(h)}}$ denotes the restriction to $S_{\mathrm{in}(h)}$. Fix a vertex $k$. We have

$$
\begin{aligned}
& \sum_{h \in H: k=\operatorname{in}(h)} \operatorname{tr}\left(B_{h}^{S}\left(B_{h}^{S}\right)^{\dagger}-\left(B_{\bar{h}}^{S}\right)^{\dagger} B_{\bar{h}}^{S}\right) \\
& =\sum_{h \in H: k=\operatorname{in}(h)} \operatorname{tr}\left(\left.B_{h} \pi_{\text {out }(h)} B_{h}^{\dagger}\right|_{S_{k}}-\left.\pi_{k} B_{h}^{\dagger} B_{\bar{h}}\right|_{S_{k}}\right) \\
& =\sum_{h \in H: k=\operatorname{in}(h)} \operatorname{tr}\left(\pi_{k}\left(\left.B_{h}\left(\pi_{\text {out }(h)}-1\right) B_{h}^{\dagger}\right|_{S_{k}}-\left.\pi_{k} i_{k} i_{k}^{\dagger}\right|_{S_{k}}-\zeta_{\mathbb{R}}^{(k)} 1_{S_{k}}\right)\right. \\
& =-\sum_{h \in H: k=\operatorname{in}(h)} \operatorname{tr}\left(\pi_{k} B_{h}\left(1-\pi_{\text {out }(h)}\right)\left\{\pi_{k} B_{h}\left(1-\pi_{\text {out }(h)}\right)\right\}^{\dagger}\right) \\
& \quad-\operatorname{tr}\left(\pi_{k} i_{k}\left(\pi_{k} i_{k}\right)^{\dagger}\right)-\zeta_{\mathbb{R}}^{(k)} \operatorname{dim} S_{k},
\end{aligned}
$$


where we have used the real ADHM equation and

$$
j_{k} \pi_{k}=0, \quad \pi_{k}^{2}=\pi_{k}, \quad \pi_{k}^{\dagger}=\pi_{k} .
$$

The above is nonpositive. On the other hand, the summation of the above for all $k$ is 0 . Hence $\operatorname{dim} S_{k}=0$ for all $k$. This shows the condition (1).

Next we show that the converse direction $(1) \Rightarrow(2)$. Suppose $m=(B, i, j) \in$ $\mu_{\mathbb{C}}^{-1}\left(-\zeta_{\mathbb{C}}\right)$ satisfies the condition (1). Following the idea of [Ki], consider the gradient flow of $f=\left\|\mu_{\mathbb{R}}+\zeta_{\mathbb{R}}\right\|^{2}$ starting from $m$. Then it can be shown that the path $m^{t}$ is contained in the $G_{\mathbf{v}}^{\mathbb{C}}$-orbit. Hence it can be written $m^{t}=g^{t} \cdot m$ for some $g^{t} \in G_{\mathbf{v}}^{\mathbb{C}}$. One can check that $m^{t}$ stays in a compact set for $t \in[0, \infty)$. By the work of Neeman [Nee] (see also Schwarz [Sc]), $g^{t} . m$ converges to $m^{\infty}=\left(B^{\infty}, i^{\infty}, j^{\infty}\right)$ as $t \rightarrow \infty$.

The limit $m^{\infty}$ is a critical point of $f$, and hence by $[\mathrm{Ki}, 3.1]$ the vector field generated by $\mu_{\mathbb{R}}\left(m^{\infty}\right)+\zeta_{\mathbb{R}} \in \mathfrak{g}_{\mathbf{v}}$ vanishes at $m^{\infty}$.

Let us write $\mu_{\mathbb{R}}\left(m^{\infty}\right)+\zeta_{\mathbb{R}}=\left(\eta_{k}\right)_{k} \in \mathfrak{g}_{\mathbf{v}}=\bigoplus_{k} \mathfrak{u}\left(V_{k}\right)$. The vanishing of the vector field is equivalent to

$$
\eta_{\text {in }(h)} B_{h}^{\infty}-B_{h}^{\infty} \eta_{\text {out }(h)}=0, \quad \eta_{k} i_{k}^{\infty}=0, \quad j_{k}^{\infty} \eta_{k}=0 \quad \text { for any } h, k
$$

Denote by $V_{k}(\lambda)$ the eigenspace of $\eta_{k}$ with the eigenvalue $\lambda$. The above implies

$$
\begin{gathered}
B_{h}^{\infty}\left(V_{\text {out }(h)}(\lambda)\right) \subset V_{\text {in }(h)}(\lambda) \quad \text { for any } \lambda, \\
i_{k}^{\infty}\left(W_{k}\right) \subset V_{k}(0), \quad j_{k}^{\infty}\left(V_{k}(\lambda)\right)=0 \quad \text { for } \lambda \neq 0 .
\end{gathered}
$$

In particular, the restriction of $\left(B^{\infty}, i^{\infty}, j^{\infty}\right)$ to $\left(V_{k}(0)\right)_{k}$ is a solution of $\mu=-\left(\zeta_{\mathbb{R}}, 0\right)$. For $\lambda \neq 0$, we have

$$
\lambda \operatorname{dim} V_{k}(\lambda)=\operatorname{tr}\left(\left.\eta_{k}\right|_{V_{k}(\lambda)}\right)=\left.\frac{i}{2} \sum_{h \in H: k=\operatorname{in}(h)} \operatorname{tr}\left(B_{h} B_{h}^{\dagger}-B_{h}^{\dagger} B_{\bar{h}}\right)\right|_{V_{k}(\lambda)}+\zeta_{\mathbb{R}}^{(k)} \operatorname{dim} V_{k}(\lambda)
$$

Here $\left.\right|_{V_{k}(\lambda)}$ denotes the restriction of linear maps to $V_{k}(\lambda)$. Summing up with respect to $k$, we get

$$
\lambda \sum_{k} \operatorname{dim} V_{k}(\lambda)=\frac{i}{2} \sum_{k} \zeta_{\mathbb{R}}^{(k)} \operatorname{dim} V_{k}(\lambda)
$$

In particular, the condition (3.4) implies $i \lambda<0$.

We study the relation between $m^{\infty}$ and $m$ to show that they are in the same $G_{\mathbf{v}}^{\mathbb{C}}$-orbit and $\eta_{k}=0$. If

$$
\left\|g^{t}\right\|^{2} \stackrel{\text { def. }}{=} \sum_{k} \operatorname{tr}\left(g_{k}^{t}\left(g_{k}^{t}\right)^{\dagger}\right) \rightarrow 0 \quad \text { as } t \rightarrow \infty
$$


we have that $j_{k}=j_{k}\left(g_{k}^{t}\right)^{-1} g_{k}^{t}$ converges to $j_{k}^{\infty} \times 0=0$. Then, setting $S=V$, we have a contradiction to the condition (1). Hence $\left\|g^{t}\right\|$ is bounded from below.

Consider the normalized endomorphism $h^{t}$ of $g^{t}$ :

$$
h^{t} \stackrel{\text { def. }}{=} \frac{g^{t}}{\left\|g^{t}\right\|} \in \bigoplus_{k} \operatorname{End}\left(V_{k}\right)
$$

Then we have $\left\|h^{t}\right\|=1$. Taking a subsequence $\left\{t_{i}\right\}$, we may assume that $h^{t_{i}}$ converges to an endomorphism $h^{\infty}$ as $i \rightarrow \infty$.

We have

$$
h_{\mathrm{in}(h)}^{\infty} B_{h}=B_{h}^{\infty} h_{\mathrm{out}(h)}^{\infty}, \quad h_{k}^{\infty} i_{k}=i_{k}^{\infty} \lim _{i \rightarrow \infty} \frac{1}{\| g^{t_{i} \|}}, \quad j_{k}^{\infty} h_{k}^{\infty}=j_{k} \lim _{i \rightarrow \infty} \frac{1}{\| g^{t_{i} \|}}
$$

First suppose that $\left\|g^{t_{i}}\right\|$ converges as $i \rightarrow \infty$. Then the subspaces $S_{k}=\operatorname{ker} h_{k}^{\infty}$ of $V_{k}$ are invariant under $B_{h}$ and in the kernel of $j_{k}$. Hence the condition (1) implies $S_{k}=0$ for all $k$. This means the $h_{k}^{\infty}$ 's are invertible, so $m$ and $m^{\infty}$ are in the same $G_{\mathrm{v}}^{\mathbb{C}}$-orbit. In particular, $m^{\infty}$ also satisfies the above condition (1), so we must have $V_{k}=V_{k}(0)$ and $m^{\infty}$ contained in $\mu_{\mathbb{R}}^{-1}\left(-\zeta_{\mathbb{R}}\right)$, as required.

Next suppose $\left\|g^{t_{i}}\right\| \rightarrow \infty$. Then we have

$$
j_{k}^{\infty} h_{k}^{\infty}=0
$$

We may also assume that

$$
0<\left.\frac{d}{d t}\left\|g^{t}\right\|^{2}\right|_{t=t_{i}} \quad \text { for any } i
$$

Since $g^{t} . m$ is a gradient flow, we have $(d / d t) g^{t}=2 i\left(\mu_{\mathbb{R}}\left(g^{t} \cdot m\right)+\zeta_{\mathbb{R}}\right) g^{t}$. (Recall that our moment map differs from Kirwan's one by sign.) Hence

$$
\frac{d}{d t}\left\|g^{t}\right\|^{2}=4 i \sum_{k} \operatorname{tr}\left(\left(g_{k}^{t}\right)^{\dagger}\left(\mu_{\mathbb{R}}\left(g^{t} \cdot m\right)+\zeta_{\mathbb{R}}\right)_{k} g_{k}^{t}\right)
$$

Dividing by $\left\|g^{t}\right\|^{2}$ and passing to the limit, we have

$$
0 \leq 4 i \sum_{k} \operatorname{tr}\left(\left(h_{k}^{\infty}\right)^{\dagger} \eta_{k} h_{k}^{\infty}\right)
$$

Since all eigenvalues of $i \eta_{k}$ are nonpositive, this inequality implies that the image of $h_{k}^{\infty}$ is contained in the 0 -eigenspace $V_{k}(0)$. Then $S_{k}^{\prime} \stackrel{\text { def. }}{=} \operatorname{im} h_{k}^{\infty} \subset V_{k}(0)$ satisfies

$$
\left.B_{h}^{\infty}\right|_{V_{\text {out }(h)}(0)}\left(S_{\text {out }(h)}^{\prime}\right) \subset S_{\text {in }(h)}^{\prime},\left.\quad j_{k}^{\infty}\right|_{V_{k}(0)}\left(S_{k}^{\prime}\right)=0 .
$$


Since the restriction of $\left(B^{\infty}, i^{\infty}, j^{\infty}\right)$ to $\left(V_{k}(0)\right)_{k}$ is a solution of $\mu=-\zeta$ and hence satisfies condition (1), $S_{k}^{\prime}$ must be zero. Hence $h^{\infty}=0$ and contradicts with $\left\|h^{\infty}\right\|=1$.

When the graph is of affine Dynkin type, we have a gauge-theoretic definition of $H_{\zeta}^{\mathrm{s}}$. The rest of this section is devoted to introducing it. This part is not used in the rest of the paper, and so the reader who is not interested in gauge-theoretic interpretation can go on to the next section.

Consider the complex (2.14). The key observation is that $\tau \sigma=0$ follows from the complex ADHM equation; it does not require the real ADHM equation. Hence we can define a holomorphic vector bundle $\operatorname{Ker} \tau / \operatorname{Im} \sigma$ when $(B, i, j)$ satisfies the complex ADHM equation. In fact, the proof of [KN] gives us the following.

Proposition 3.6. Assume $\zeta=\left(\zeta_{\mathbb{R}}, \zeta_{\mathbb{C}}\right)$ satisfies the condition (2.13). Suppose that $(B, i, j)$ satisfies the following two conditions:

(i) (nondegeneracy) $\sigma$ is injective and $\tau$ is surjective,

(ii) (the complex $A D H M$ equation) $\mu_{\mathbb{C}}(B, i, j)=-\zeta_{\mathbb{C}}$.

Then the induced connection $A$ on $E=\operatorname{Coker}\left(\sigma, \tau^{\dagger}\right)$ is compatible with a holomorphic structure $\operatorname{Ker} \tau / \operatorname{Im} \sigma$. Moreover, the connection has the asymptotic behavior

$$
A-A_{0}=O\left(r^{-3}\right), \quad \nabla_{A_{0}}\left(A-A_{0}\right)=O\left(r^{-4}\right), \ldots
$$

where $A_{0}$ is a flat connection defined on the end $X_{\zeta} \backslash K$ and $\nabla_{A_{0}}$ is a covariant derivative associated with $A_{0}$. Conversely, every connection which satisfies the above asymptotic condition and is compatible with a holomorphic structure (i.e., $\bar{\partial}_{A} \bar{\partial}_{A}=0$ ) arises by this construction.

Let $\hat{H}_{\zeta}^{\text {s }}$ be the set of data satisfying (i), (ii). Since we deal with only the complex ADHM equation, the "gauge" group for the ADHM data is enlarged: $\hat{H}_{\zeta}^{\text {s }}$ is invariant under the action of the complexification $G_{\mathbf{v}}^{\mathbb{C}}$. We have a natural holomorphic map $\mathfrak{M}_{\zeta}^{\text {reg }} \rightarrow \hat{H}_{\zeta}^{\mathrm{s}} / G_{\mathbf{v}}^{\mathbb{C}}$. Proposition 3.6 gives a bijection between $\hat{H}_{\zeta}^{\mathrm{s}} / G_{\mathbf{v}}^{\mathbb{C}}$ and the framed moduli space $\mathfrak{M}_{\zeta}^{\text {hol }}(E)$ of connections on a hermitian vector bundle $E$ over $X_{\zeta}$ which satisfy $\bar{\partial}_{A} \bar{\partial}_{A}=0$ and the above asymptotic behavior. On the other hand, we have a bijective correspondence between $\mathfrak{M}_{\zeta}^{\text {reg }}$ and the framed moduli space $\mathfrak{M}_{\zeta}(E)$ of instantons by Proposition 2.4. We have a map $\mathfrak{M}_{\zeta}(E) \rightarrow \mathfrak{M}_{\zeta}^{\text {hol }}(E)$ since anti-self-dual connections satisfy $\bar{\partial}_{A} \bar{\partial}_{A}=0$. We thus have the following commutative diagram:

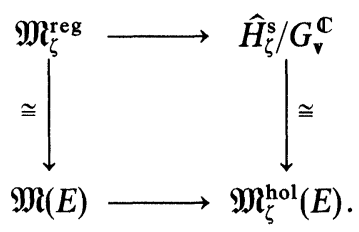


Now the Hitchin-Kobayashi correspondence over the ALE space $X_{\zeta}$ states that the below horizontal arrow is a bijection. This result was proved by Bando [Ba]. (He solved Einstein-hermitian equations on more general noncompact manifolds.) Hence we have the holomorphic description of $\mathfrak{M}_{\zeta}$.

THEOREM 3.7. If $\zeta=\left(\zeta_{\mathbb{R}}, \zeta_{\mathbb{C}}\right)$ satisfies (2.13), we have an isomorphism between complex manifolds:

$$
\mathfrak{M}_{\zeta}^{\mathrm{reg}} \cong \hat{H}_{\zeta}^{\mathrm{s}} / G_{\mathrm{v}}^{\mathbb{C}}
$$

In particular, $H_{\zeta}^{\mathrm{s}}=\hat{H}_{\zeta}^{\mathrm{s}}$, when the underlying graph is of Dynkin type (see Remark 2.16).

Remark 3.8. Since $\mathfrak{M}_{\zeta}^{\text {reg }}$ and $\hat{H}_{\zeta}^{\mathrm{s}} / G_{v}^{\mathbb{C}}$ are both finite-dimensional objects, it is desirable to have a direct proof which does not rely on Bando's analytic result. In fact, the Hitchin-Kobayashi correspondence on $\mathbb{R}^{4}$ was proved by Donaldson in that way [Do].

4. The resolution of singularities. Consider $\zeta=\left(0, \zeta_{\mathbb{C}}\right)$ and $\tilde{\zeta}=\left(\zeta_{\mathbb{R}}, \zeta_{\mathbb{C}}\right)$. The inclusion $\mu_{\mathbb{R}}^{-1}\left(-\zeta_{\mathbb{R}}\right) \cap \mu_{\mathbb{C}}^{-1}\left(-\zeta_{\mathbb{C}}\right) \rightarrow \mu_{\mathbb{C}}^{-1}\left(-\zeta_{\mathbb{C}}\right)$ and the algebro-geometric quotient $\operatorname{map} \mu_{\mathbb{C}}^{-1}\left(-\zeta_{\mathbb{C}}\right) \rightarrow \mu_{\mathbb{C}}^{-1}\left(-\zeta_{\mathbb{C}}\right) / / G_{\mathfrak{v}}^{\mathbb{C}}=\mathfrak{M}_{\left(0, \zeta_{\mathbb{C}}\right)}$ together give a map

$$
\pi: \mathfrak{M}_{\tilde{\zeta}} \rightarrow \mathfrak{M}_{\zeta} .
$$

This is holomorphic with respect to $I$.

We assume that $\tilde{\zeta}$ is generic. So $\mathfrak{M}_{\tilde{\zeta}}$ is smooth by Theorem 2.8 .

THEOREM 4.1. If $\mathfrak{M}_{\zeta}^{\mathrm{reg}}$ is nonempty, $\pi$ is a resolution of singularities (though the singular locus may be empty). That is,

(1) $\pi: \mathfrak{M}_{\tilde{\zeta}} \rightarrow \mathfrak{M}_{\zeta}$ is proper.

(2) $\pi$ induces an isomorphism $\pi^{-1}\left(\mathfrak{M}_{\zeta}^{\text {reg }}\right) \simeq \mathfrak{M}_{\zeta}^{\text {reg }}$.

(3) $\pi^{-1}\left(\mathfrak{M}_{\zeta}^{\mathrm{reg}}\right)$ is a dense subset in $\mathfrak{M}_{\tilde{\zeta}}$.

We give proofs of statements (1) and (2) in this section. The proof of (3) will be given in the end of $\S 6$. The statements (1) and (2) hold without the assumption $\mathfrak{M}_{\zeta}^{\text {reg }} \neq \varnothing($ even without the genericity of $\tilde{\zeta})$.

Proof. (1) Let $K \subset \mathfrak{M}_{\zeta}$ be a compact set and assume that $\pi^{-1}(K)$ is noncompact. There exists a sequence $\left\{x_{i}\right\} \subset \pi^{-1}(K)$ which has no convergent subsequences. If we take a representative $m_{i} \in \mu^{-1}(-\tilde{\zeta})$ of $x_{i}$, we have $\left\|m_{i}\right\| \rightarrow \infty$ as $i \rightarrow \infty$. Consider

$$
m_{i}^{\prime} \stackrel{\text { def. }}{=} \frac{m_{i}}{\left\|m_{i}\right\|} \in \mu^{-1}\left(-\left\|m_{i}\right\|^{-2} \tilde{\zeta}\right)
$$

Since $\left\|m_{i}^{\prime}\right\|=1$, we may assume that $m_{i}^{\prime}$ converges to $m_{\infty}^{\prime} \in \mu^{-1}(0)$, which satisfies $\left\|m_{\infty}^{\prime}\right\|=1$. 
If $n_{i}$ is a representative of $\pi\left(x_{i}\right)$,

$$
n_{i}^{\prime} \stackrel{\text { def. }}{=} \frac{n_{i}}{\left\|m_{i}\right\|}
$$

is a representative of $\pi\left(\left[m_{i}^{\prime}\right]\right)$. ([•] denotes the $G_{\mathbf{v}}$-orbit of $\bullet$ considered as a point in $\mathfrak{M}_{\tilde{\zeta}}$ or $\mathfrak{M}_{\zeta}$.) This statement follows from properties of the algebro-geometric quotient map: $\pi([m])=[n]$ if and only if the $G_{\mathbf{v}}^{\mathbb{C}}$-orbit $G_{\mathbf{v}}^{\mathbb{C}} n$ is the unique closed orbit contained in the closure $\overline{G_{\mathbf{v}}} \overline{\mathbb{C}}$ of the orbit $G_{\mathbf{v}}^{\mathbb{C}} m$, since $K$ is compact and $\left[n_{k}\right] \in K, n_{i}^{\prime}$ converges to 0 . This implies $\pi\left(\left[m_{\infty}^{\prime}\right]\right)=0$; and so $0 \in \overline{G_{\mathbf{v}}^{\mathbb{C}} m_{\infty}^{\prime}}$. But the orbit $G_{\mathbf{v}}^{\mathbb{C}} m_{\infty}^{\prime}$ is closed since $m_{\infty}^{\prime}$ is in $\mu^{-1}(0)$. (This follows from the general properties of moment maps and algebro-geometric quotients. See [Ne, Theorem 2.2].) This means $m_{\infty}^{\prime}=0$ and contradicts with $\left\|m_{\infty}^{\prime}\right\|=1$.

(2) This statement is proved in [Kr1, 3.10] for ALE spaces. The proof works also for our case if we use (1) instead of [Kr1, 3.11].

Theorem 4.1 (we only need (1), (2)) has an interesting corollary.

COROLlARY 4.2. If $\zeta$ and $\zeta^{\prime}$ are generic, $\mathfrak{M}_{\zeta}$ and $\mathfrak{M}_{\zeta^{\prime}}$ are diffeomorphic.

Proof. We use notation preserving the symmetry of three complex structures $I, J, K$, i.e., $\zeta_{\mathbb{R}}=\zeta_{I}, \zeta_{\mathbb{C}}=\zeta_{J}+i \zeta_{K}$ where $\zeta_{I}, \zeta_{J}, \zeta_{K} \in Z$. Set

$$
\begin{gathered}
\zeta=\left(\zeta_{I}, \zeta_{J}, \zeta_{K}\right), \quad \zeta^{\prime}=\left(\zeta_{I}^{\prime}, \zeta_{J}^{\prime}, \zeta_{K}^{\prime}\right), \quad \eta=\left(\zeta_{I}^{\prime}, \zeta_{J}, \zeta_{K}\right), \\
\sigma=\left(\zeta_{I}^{\prime}, \zeta_{J}^{\prime}, \zeta_{K}\right), \quad \xi=\left(0, \zeta_{J}, \zeta_{K}\right) .
\end{gathered}
$$

As in [Kr1, proof of Corollary 3.12$]$, we may assume that $\left(0,0, \zeta_{K}\right)$ and $\left(\zeta_{I}^{\prime}, \zeta_{J}^{\prime}, 0\right)$ are generic by choosing a new orthonormal basis for $\mathbb{R}^{3}$, if necessary. Then $\mathfrak{M}_{\xi}$ is nonsingular and maps $\pi_{1}: \mathfrak{M}_{\zeta} \rightarrow \mathfrak{M}_{\xi}, \pi_{2}: \mathfrak{M}_{\eta} \rightarrow \mathfrak{M}_{\xi}$ are isomorphisms. In particular, $\mathfrak{M}_{\zeta}$ and $\mathfrak{M}_{\eta}$ are diffeomorphic. Changing the roles of $I, J$, and $K$, we can prove that $\mathfrak{M}_{\eta}, \mathfrak{M}_{\sigma}$, and $\mathfrak{M}_{\zeta^{\prime}}$ are all diffeomorphic.

5. The $\mathbb{C}^{*}$-action, the Lagrangian variety $\mathfrak{L}$, and the fixed-point variety $\mathfrak{F}$. Throughout this section we fix a real parameter $\zeta_{\mathbb{R}}$ so that $\left(\zeta_{\mathbb{R}}, 0\right)$ is generic.

Consider complex analytic spaces

$$
\mathscr{M}_{\zeta_{\mathbb{R}}} \stackrel{\text { def. }}{=} \bigcup_{\zeta_{\mathbb{R}} \in Z_{v} \otimes \mathbb{C}} \mathfrak{M}_{\left(\zeta_{\mathbb{R}}, \zeta_{\mathbb{C}}\right)}, \quad \mathscr{M}_{0} \stackrel{\text { def. }}{=} \bigcup_{\zeta_{\mathbb{C}} \in Z_{\mathfrak{v}} \otimes \mathbb{C}} \mathfrak{M}_{\left(0, \zeta_{\mathbb{C}}\right)}
$$

These spaces have natural $S^{1}$-actions defined by

$$
\left(B_{h}, i_{k}, j_{k}\right) \mapsto\left(t^{(1-\varepsilon(h)) / 2} B_{h}, i_{k}, t j_{k}\right) \quad \text { for } t \in S^{1} .
$$

Namely, the components in $\mathbf{M}_{\bar{\Omega}}$ are multiplied by $t$, while components in $\mathbf{M}_{\boldsymbol{\Omega}}$ are unchanged. The real ADHM equation $\mu_{\mathbb{R}}(B, i, j)=-\zeta_{\mathbb{R}}$ is preserved by 
the action, while the complex moment map is transformed as $\mu_{\mathbb{C}}(t .(B, i, j))=$ $t \mu_{\mathbb{C}}(B, i, j)$. We list its important properties.

THEOREM 5.1. The $S^{1}$-action on $\mathscr{M}_{\zeta_{\mathbb{R}}}$ has the following properties:

(1) The natural projection map $\mathscr{M}_{\zeta_{\mathbb{R}}} \rightarrow Z \otimes \mathbb{C}$ is equivariant; here we make $S^{1}$ act on the vector space $Z \otimes \mathbb{C}$ with weight 1 . (In particular, $\mathfrak{M}_{\left(\zeta_{\mathbb{R}}, 0\right)}$ admits an $S^{1}$-action.)

(2) It preserves the complex structure I and the metric.

(3) The holomorphic symplectic form $\omega_{\mathbb{C}}$ transforms as $\omega_{\mathbb{C}} \rightarrow t \omega_{\mathbb{C}}$.

(4) The corresponding moment map

$$
F([B, i, j])=\sum_{h \in \bar{\Omega}}\left\|B_{h}\right\|^{2}+\sum_{k}\left\|j_{k}\right\|^{2}
$$

is proper.

(5) The action is extended to a holomorphic (with respect to I) $\mathbb{C}^{*}$-action. If we use the holomorphic description $\left.\mathfrak{M}_{\left(\zeta_{\mathbb{R}}, \zeta_{\mathbb{C}}\right)}=H_{\left(\zeta_{\mathbb{R}}, \zeta_{\mathbb{C}}\right)}^{\mathrm{s}}\right) G_{\mathbf{v}}^{\mathbb{C}}$, the $\mathbb{C}^{*}$-action is given by

$$
G_{\mathbf{v}}^{\mathbb{C}}\left(B_{h}, i_{k}, j_{k}\right) \mapsto G_{\mathbf{v}}^{\mathbb{C}}\left(t^{(1-\varepsilon(h)) / 2} B_{h}, i_{k}, t j_{k}\right) .
$$

Proof. The statements (1), (2), and (3) are clear. (4) Suppose that there exists a sequence $\left\{x_{i}\right\} \subset \mathscr{M}_{\zeta_{\mathbb{R}}}$ such that

(a) it has no covergent subsequences,

(b) $F\left(x_{i}\right)$ is bounded from above by a constant independent of $i$.

Take a representative $m_{i}$ of $x_{i}$. As $\left\|m_{i}\right\| \rightarrow \infty$, we take a normalized sequence $m_{i} /\left\|m_{i}\right\|$ as in Theorem 4.1 . Then (after taking the subsequence) we may assume that $m_{i} /\left\|m_{i}\right\|$ converges to $m_{\infty}$ with

(c) $\left\|m_{\infty}\right\|=1$,

(d) $\mu_{\mathbb{R}}\left(m_{\infty}\right)=0$,

(e) $F\left(m_{\infty}\right)=0$, i.e., the $\mathbf{M}_{\bar{\Omega}}$-component of $m_{\infty}$ is zero.

Since we assume $\Omega$ has no cycle, there is a source vertex $k$, i.e., $l \rightarrow k \notin \Omega$ for any $l$. Then (e) and the real ADHM equation (d) imply that the components of $m_{\infty}$ which map into $V_{k}$ vanish. Hence $m_{\infty}$ can be considered as a datum for the graph with the vertex $k$ removed. By induction, we have $m_{\infty}=0$, which contradicts with (c).

(5) This statement is not trivial; the real ADHM equation is not preserved by the above action when $t$ is not necessary in $S^{1}$. Since $\left(\zeta_{\mathbb{R}}, 0\right)$ is generic, $\mathfrak{M}_{\left(\zeta_{\mathbb{R}}, \zeta_{\mathbb{C}}\right.}$ is nonsingular for any $\zeta_{\mathbb{C}} \in Z_{\mathbf{v}} \otimes \mathbb{C}$. If $V$ is the generating vector field of the $S^{1}$ action, then we have

$$
\operatorname{grad} F=2 I V
$$

The above (4), in particular, implies that the vector field $I V$ is complete. Hence the 1-parameter group of transformations which induces $I V$ gives the required $\mathbb{C}^{*}$-action. 
Let $\mathscr{M}_{\zeta_{\mathbb{R}}}=\bigcup_{\zeta_{\mathbb{C}} \in Z_{\mathbf{v}} \otimes \mathbb{C}} H_{\left(\zeta_{\mathbb{R}}\right.}^{\mathrm{s}}, \zeta_{\mathbb{C}} / G_{\mathbf{v}}^{\mathbb{C}}$ be the holomorphic description. Take a representative $(B, i, j)\left(\operatorname{resp} .\left(B^{\prime}, i^{\prime}, j^{\prime}\right)\right)$ of $x$ (resp. $\left.t . x\right)$. Then $\left(B^{\prime}, i^{\prime}, j^{\prime}\right)$ is contained in the closure of the $G_{v}^{\mathbb{C}}$-orbit through $\left(t^{(1-\varepsilon(h)) / 2} B_{h}, i_{k}, t j_{k}\right)$. But $\left(B^{\prime}, i^{\prime}, j^{\prime}\right)$ is contained in $\mu^{-1}\left(-\zeta_{\mathbb{R}},-t \zeta_{\mathbb{C}}\right)$, its $G_{\mathrm{v}}^{\mathbb{C}}$-orbit has the maximal dimension, and it is not contained in any other orbits. Hence $\left(B^{\prime}, i^{\prime}, j^{\prime}\right)$ is contained in $G_{\mathbf{v}}^{\mathbb{C}}\left(t^{(1-\varepsilon(h)) / 2} B_{h}, i_{k}, t j_{k}\right)$.

For $\mathscr{M}_{0}$, we can use the holomorphic description $\mathscr{M}_{0}=\bigcup_{\zeta_{\mathbb{C}} \in Z_{\mathbf{v}} \otimes \mathbb{C}} \mu_{\mathbb{C}}^{-1}\left(-\zeta_{\mathbb{C}}\right) / /$ $G_{\mathbf{v}}^{\mathbb{C}}$ (Theorem 3.1) to extend the $S^{1}$-action to a $\mathbb{C}^{*}$-action. Then the following is clear.

THEOREM 5.2. The map $\pi: \mathscr{M}_{\zeta_{\mathbb{R}}} \rightarrow \mathscr{M}_{0}$ given in $\S 4$ is $\mathbb{C}^{*}$-equivariant.

Remark 5.3. (1) By [Kr1, Remark on page 682]

$$
\mathscr{X}_{\zeta_{\mathbb{R}}} \stackrel{\text { def. }}{=} \bigcup_{\zeta_{\mathbb{C}} \in Z \otimes \mathbb{C}} X_{\left(\zeta_{\mathbb{R}}, \zeta_{\mathbb{C}}\right)} \rightarrow \bigcup_{\zeta_{\mathbb{C}} \in Z \otimes \mathbb{C}} X_{\left(0, \zeta_{\mathbb{C}}\right)} \stackrel{\text { def. }}{=} \mathscr{X}_{0}
$$

is the simultaneous resolution of the semiuniversal deformation of $X_{0}=\mathbb{C}^{2} / \Gamma$ constructed by Brieskorn, Slodowy, and Tjurina. Slodowy defined $\mathbb{C}^{*}$-actions on $\mathscr{X}_{\zeta_{\mathbb{R}}}, \mathscr{X}_{0}$ under which the above map is equivariant [S1, 4.2]. Kronheimer observed that the $\mathbb{C}^{*}$-action on $\mathscr{X}_{0}$ coincides with Slodowy's one using the holomorphic description $\mathscr{X}_{0}=\mu_{\mathbb{C}}^{-1}(Z \otimes \mathbb{C}) / / G_{\mathbf{n}}^{\prime \mathbb{C}}[\mathrm{Kr} 1$, page 681$]$. Our action on $\mathscr{X}_{\zeta_{\mathbb{R}}}$ coincides with Slodowy's action.

(2) The fixed point set of the $\mathbb{C}^{*}$-action in the quiver variety $\mathfrak{M}_{0}$ with the parameter $\zeta=0$ consists of the single point 0 . (If $[(B, i, j)] \in \mathfrak{M}_{0}$ is a fixed point of $\mathbb{C}^{*}$-action, its $\mathbf{M}_{\overline{\mathbf{\Omega}}}$-component is zero. Hence the argument in the proof of (5.1) (4) shows $(B, i, j)=0$.) Since $\pi$ is equivariant, the fixed point set in $\mathfrak{M}_{\left(\zeta_{\mathbb{R}}, 0\right)}$ is contained in $\pi^{-1}(0)$.

We introduce two very important subvarieties in $\mathfrak{M}_{\left(\zeta_{\mathbb{R}}, 0\right)}$.

Definition 5.4. (1) An element $(B, i, j) \in \mu^{-1}\left(-\zeta_{\mathbb{R}}, 0\right)$ is said to be nilpotent if $[(B, i, j)] \in \pi^{-1}(0)$. The subvariety $\pi^{-1}(0)$ consists of nilpotent elements and is denoted by $\mathfrak{L}$. When we want to emphasize the dimensions, we use the notation $\mathfrak{Q}(\mathbf{v}, \mathbf{w})$.

(2) Let $\mathfrak{F}=\mathfrak{F}(\mathbf{v}, \mathbf{w})$ be the fixed-point set of the $S^{1}$-action. By definition, it is the fixed-point set of the $\mathbb{C}^{*}$-action.

The argument by Slodowy $[S 1,4.3]$, applied to the map $\pi: \mathfrak{M}_{\left(\zeta_{\mathbb{R}}, 0\right)} \rightarrow \mathfrak{M}_{0}$, shows the following.

COROLLARY 5.5. $\mathfrak{M}_{\left(\zeta_{\mathbb{R}}, 0\right)}$ is homotopy equivalent to $\mathfrak{L}=\pi^{-1}(0)$.

The fixed-point set $\mathfrak{F}$ is a nonsingular subvariety of $\mathfrak{M}_{\left(\zeta_{\mathbb{R}}, 0\right)}$. We now show that the homology of $\mathfrak{M}_{\left(\zeta_{\mathbb{R}}, 0\right)}$ is determined by the homology of the connected components $\mathfrak{F}_{1}, \ldots, \mathfrak{F}_{p}$ of $\mathfrak{F}$ and the action of $S^{1}$ near $\mathfrak{F}$. The method is due to Frankel [Fr]. Recall that the moment map $F$ for the $S^{1}$-action is a proper function on $\mathfrak{M}_{\left(\zeta_{\mathbb{R}}, 0\right)}$ by Theorem 5.1(4). It is known that the moment map for a circle action 
on a compact Kähler manifold is a perfect nondegenerate Morse function in the sense of Bott [Bo] (see [AB], [Ki]). The proof also works if every path of steepest descent for $F$ stays in a compact set. If $V$ is the generating vector field, we have

$$
\operatorname{grad} F=2 I V,
$$

where $I$ is the complex structure. Thus the critical points are the fixed points of the $S^{1}$-action, that is, points in $\mathfrak{F}$. Let $x \in \mathfrak{F}_{\alpha}$. The $S^{1}$ acts on the tangent space $T_{x} \mathfrak{M}_{\left(\zeta_{\mathbb{R}}, 0\right)}$, which decomposes into the sum

$$
\bigoplus_{m} H^{m}
$$

of complex subspaces where $S^{1}$ acts on $H^{m}$ with weight $m$. The tangent space $T_{x} \mathfrak{F}_{\alpha}$ is equal to $H^{0}$. The hessian of $F$ at $x$ acts on $H^{m}$ as multiplication by $m$ (see [At]). Hence the index at $x$ is given by

$$
m_{\alpha} \stackrel{\text { def. }}{=} 2 \sum_{m<0} \operatorname{dim}_{\mathbb{C}} H^{m}
$$

LEMMA 5.6. We have

$$
m_{\alpha}+\operatorname{dim}_{\mathbb{R}} \mathfrak{F}_{\alpha}=\frac{1}{2} \operatorname{dim}_{\mathbb{R}} \mathfrak{M}_{\left(\zeta_{\mathbb{R}}, 0\right)}
$$

Proof. If we pull back the holomorphic symplectic form $\omega_{\mathbb{C}}$, it is multiplied by $t$. So $H^{m}$ and $H^{1-m}$ are dual to each other with respect to $\omega_{\mathbb{C}}$. Hence

$$
\sum_{m \leqslant 0} \operatorname{dim}_{\mathbb{C}} H^{m}=\sum_{m>0} \operatorname{dim}_{\mathbb{C}} H^{m} .
$$

This proves the assertion.

Hence we have the following.

Proposition 5.7.

$$
H_{i}\left(\mathfrak{M}_{\left(\zeta_{\mathbb{R}}, 0\right)} ; \mathbb{Z}\right) \cong H_{i}(\mathfrak{L} ; \mathbb{Z}) \cong \underset{1 \leqslant \alpha \leqslant p}{\bigoplus} H_{i-m_{\alpha}}\left(\mathfrak{F}_{\alpha} ; \mathbb{Z}\right),
$$

where $m_{\alpha}=(1 / 2) \operatorname{dim}_{\mathbb{R}} \mathfrak{M}_{\left(\zeta_{\mathbb{R}}, 0\right)}-\operatorname{dim}_{\mathbb{R}} \mathfrak{F}_{\alpha}$.

In a forthcoming article, we shall give a combinatorial algorithm for the calculation of Betti numbers using this proposition. When the graph is of type $A_{n}$, the algorithm is given in terms of Young tableaux [Na2].

The above proof also shows the following result.

THEOREM 5.8. The variety $\mathfrak{L}=\pi^{-1}(0)$ decomposes as

$$
\mathfrak{L}=\bigcup_{1 \leqslant \alpha \leqslant p}\left\{\left.x \in \mathfrak{M}_{\left(\zeta_{\mathbb{R}}, 0\right)}\right|_{t \rightarrow \infty} t . x \text { exists and belongs to } \mathfrak{F}_{\alpha}\right\} .
$$


The closure of each summand is an irreducible component of $\mathfrak{L}$. $\mathfrak{L}$ is a Lagrangian subvariety of $\mathfrak{M}_{\left(\zeta_{\mathbb{R}}, 0\right)}$ with respect to the holomorphic symplectic form $\omega_{\mathbb{C}}$.

Proof. Since $\pi$ is equivariant and proper, $\lim _{t \rightarrow \infty} t$. $x$ exists if and only if $x \in \mathfrak{L}$. The limit is a fixed point of the $\mathbb{C}^{*}$-action, i.e., a point in $\mathfrak{F}$. Hence $\mathfrak{L}$ decomposes according to the decomposition $\mathfrak{F}=\bigcup \mathfrak{F}_{\alpha}$.

We now show that each summand is a Lagrangian submanifold. The symplectic form $\omega_{\mathbb{C}}$ is multiplied by $t$, by the $\mathbb{C}^{*}$-action. Hence $\omega_{\mathbb{C}}(v, w)$ must vanish if $v$, $w \in T_{x} \mathfrak{L}$. This means that $T_{x} \mathfrak{L}$ is an isotropic subspace.

Suppose $x \in \mathfrak{F}$ and let

$$
T_{x} \mathfrak{M}_{\left(\zeta_{\mathbb{R}}, 0\right)}=\bigoplus_{m} H^{m}
$$

be the weight space decomposition. Then we have

$$
T_{x} \mathfrak{L}=\bigoplus_{m \leqslant 0} H^{m}
$$

Lemma 5.6 shows that the dimension is half of that of $\mathfrak{M}_{\left(\zeta_{\mathbb{R}}, 0\right)}$. Hence $\mathfrak{L}$ is a Lagrangian subvariety.

We give a property of $\mathfrak{L}$ which will be needed in $\S 10$.

LEMMA 5.9. Assume $\zeta_{\mathbb{R}}$ satisfies the condition (3.4). Then

$$
\mathfrak{L}=\left\{[(B, i, j)] \in \mathfrak{M}_{\left(\zeta_{\mathbb{R}}, 0\right)} \mid i=0, \overline{G_{\mathbf{v}}^{\mathbb{C}} B} \ni 0\right\}
$$

Proof. The subvariety $\mathfrak{L}$ is defined by $\mathfrak{L}=\pi^{-1}(0)$. A point $[(B, i, j)] \in \mathfrak{M}_{\left(\zeta_{\mathbb{R}}, 0\right)}$ is mapped to 0 by $\pi$ if and only if the $0 \in \overline{G_{\mathbf{v}}^{\mathbb{C}}(B, i, j)}$. By Hilbert's criterion (see $[\mathrm{Ne}, \S \S 3,4])$, this happens if and only if there is a 1 -parameter subgroup $\lambda: \mathbb{C}^{*} \rightarrow$ $G_{\mathbf{v}}^{\mathbb{C}}$ such that it is a complexification of a closed real 1-parameter subgroup $\lambda: S^{1} \rightarrow G_{\mathrm{v}}$ and $0 \in \overline{\lambda\left(\mathbb{C}^{*}\right)(B, i, j)}$. Denote the $\lambda$-weight decomposition of $V_{k}$ by

$$
V_{k}=\bigoplus_{m \in \mathbb{Z}} V_{k}^{m}
$$

where $S^{1}$ acts on $V_{k}^{m}$ with weight $m$. This induces the weight decomposition of $\mathbf{M}$,

$$
\mathbf{M}=\bigoplus_{m \in \mathbb{Z}} \mathbf{M}^{m}
$$

Then $0 \in \overline{\lambda\left(\mathbb{C}^{*}\right)(B, i, j)}$ if and only if

$$
\max \left\{m \mid \text { the } \mathbf{M}^{m} \text {-component of }(B, i, j) \text { is nonzero. }\right\}<0
$$

or $\min \left\{m \mid\right.$ the $\mathbf{M}^{m}$-component of $(B, i, j)$ is nonzero. $\}>0$. 
We may assume that the latter case occurs. Let $V_{k}^{(n)} \stackrel{\text { def. }}{=} \bigoplus_{m \leqslant n} V_{k}^{m}$. Hence

$$
0=V_{k}^{(-N)} \subset \cdots \subset V_{k}^{(n)} \subset V_{k}^{(n+1)} \subset \cdots \subset V_{k}^{(N)}=V_{k}
$$

forms a filtration of $V_{k}$. ( $N$ is sufficiently large.) Then the above condition can be rewritten as

$$
B_{h}\left(V_{\mathrm{out}(h)}^{(m)}\right) \subset V_{\mathrm{in}(h)}^{(m-1)}, \quad i_{k}\left(W_{k}\right) \subset V_{k}^{(-1)}, \quad j_{k}\left(V_{k}^{(0)}\right)=0 .
$$

(Note $S^{1}$ acts on $W_{k}$ trivially.) Let $m_{0} \stackrel{\text { def. }}{=} \min \left\{m \mid V_{k}^{(m)} \neq 0\right.$ for some $\left.k\right\}$. By definition and the above, the restriction of $B_{h}$ to $V_{\text {out }(h)}^{\left(m_{0}\right)}$ is zero. If $m_{0} \leqslant 0$, the restriction of $j_{k}$ is also zero. But it contradicts with the real ADHM equation

$$
\begin{aligned}
\sum_{h \in H: k=\mathrm{in}(h)}\left(B_{h} B_{h}^{\dagger}-B_{\bar{h}}^{\dagger} B_{\bar{h}}\right)+i_{k} i_{k}^{\dagger}-\left.j_{k}^{\dagger} j_{k}\right|_{V_{k}^{\left(m_{0}\right)}} & =\sum_{h \in H: k=\mathrm{in}(h)} B_{h} B_{h}^{\dagger}+\left.i_{k} i_{k}^{\dagger}\right|_{V_{k}^{\left(m_{0}\right)}} \\
& =-\zeta_{\mathbb{R}}^{(k)} 1_{V_{k}^{\left(m_{0}\right)}},
\end{aligned}
$$

since the left-hand side is positive semidefinite and the right-hand side is negative definite. Hence $m_{0}>0$ and $i=0$.

Conversely, suppose $i=0$ and $0 \in \overline{G_{\mathrm{v}}^{\mathbb{C}} B}$. By the Hilbert criterion, there exists a 1-parameter subgroup $\lambda: \mathbb{C}^{*} \rightarrow G_{v}^{\mathbb{C}}$ such that $0 \in \overline{\lambda\left(\mathbb{C}^{*}\right) B}$. Multiplying the diagonal matrix $t^{n}$ to $\lambda(t)$ if necessary (this does not affect the $\mathbb{C}^{*}$-action on $B$-components), we may assume that $\mathbb{C}^{*}$ acts on $V$ with positive weights. Then $0 \in \overline{G_{\mathbf{v}}^{\mathbb{C}}(B, 0, j)}$.

Remarks 5.11. (1) Let us define $\Lambda \equiv \Lambda(\mathbf{v}, \mathbf{w}) \stackrel{\text { def. }}{=}\left\{(B, i, j) \in \mu_{\mathbb{C}}^{-1}(0) \mid 0 \in \overline{G_{\mathbf{v}}^{\mathbb{C}}(B, i, j)}\right\}$. Our Lagrangian subvariety $\mathfrak{L}$ is given as a Kähler quotient of $\Lambda$ by the group $G_{\mathbf{v}}$, that is,

$$
\mathfrak{L}=\Lambda \cap \mu_{\mathbb{R}}^{-1}\left(-\zeta_{\mathbb{R}}\right) / G_{\mathbf{v}} .
$$

We also have a holomorphic description (Theorem 3.2)

$$
\mathfrak{L}=\Lambda \cap H_{\left(\zeta_{\mathbb{R}}, 0\right)}^{\mathrm{s}} / G_{\mathbf{v}}^{\mathbb{C}} .
$$

By the general property of hyper-Kähler quotients, $\mathfrak{I}$ is a Lagrangian subvariety if and only if $\Lambda \cap H_{\zeta}^{\text {s }}$ is a Lagrangian subvariety.

When $\zeta$ satisfies (3.4), we have a direct decomposition

$$
\Lambda \equiv \Lambda(\mathbf{v}, \mathbf{w})=\Lambda(\mathbf{v}, 0) \times \bigoplus_{k} \operatorname{Hom}\left(V_{k}, W_{k}\right)
$$

The variety $\Lambda(v, 0)$ was introduced by Lusztig [Lu3, 12.9]. He announced that $\Lambda(\mathbf{v}, 0)$ is a Lagrangian subvariety of $\mathbf{M}(\mathbf{v}, 0)$. Since the stability is an open condi- 
tion, our result follows from his assertion. Conversely, our result implies Lusztig's assertion. This follows from the observation that $\Lambda(\mathbf{v}, 0) \times \bigoplus_{k} \operatorname{Hom}\left(V_{k}, W_{k}\right) \subset H_{\zeta}^{\mathrm{s}}$ when the dimensions of the $W_{k}$ 's are sufficiently large.

(2) Let $\operatorname{Irr} \mathfrak{L}(\mathbf{v}, \mathbf{w})$ (resp. Irr $\Lambda(\mathbf{v}, \mathbf{w})$ ) be the set of irreducible components of $\mathfrak{I}(\mathbf{v}, \mathbf{w})$ (resp. $\Lambda(\mathbf{v}, \mathbf{w}))$. Since $H_{\zeta}^{s} \rightarrow \mathfrak{M}_{\zeta}(\mathbf{v}, \mathbf{w})$ is a principal $G_{\mathbf{v}}^{\mathbb{C}}$-bundle, Irr $\mathfrak{L}(\mathbf{v}, \mathbf{w})$ can be identified with

$$
\left\{Y \in \operatorname{Irr} \Lambda(\mathbf{v}, \mathbf{w}) \mid Y \cap H_{\zeta}^{\mathrm{s}} \neq \varnothing\right\}
$$

(3) Assume that $\zeta=\left(\zeta_{\mathbb{R}}, 0\right)$ satisfies (3.4). Let us decompose $(B, 0, j)$ into $\left(B^{\prime}, 0\right) \oplus\left(B^{\prime \prime}, j\right)$ according to $\mathbf{M}=\mathbf{M}_{\Omega} \oplus \mathbf{M}_{\bar{\Omega}}$ (see $\left.(2.1)\right)$. Then $\mu_{\mathbb{C}}(B, 0, j)=0$ if and only if $\left(B^{\prime \prime}, j\right)$ is orthogonal with respect to $\omega_{\mathbb{C}}$ to the tangent space to the $G_{\mathbf{v}}^{\mathbb{C}}$ orbit of $\left(B^{\prime}, 0\right)$ (regarded as a subspace of $\left.\mathbf{M}_{\Omega}\right)$. (Compare to [L3, 12.8(a)].) Thus $\Lambda$ is contained in the union of the conormal bundles of the various $G_{\mathbf{v}}^{\mathbb{C}}$-orbits in $\mathbf{M}_{\Omega} \cap\{i=0\}$. (M is regarded as a cotangent bundle on $\mathbf{M}_{\mathbf{\Omega}}$.)

When the graph is of Dynkin type, it can be shown that the nipotency condition in the definition of $\Lambda$ is vacuous. (See $[\mathrm{L3}, 14.2]$ or 6.7 below together with (3.1).) There are only finitely many orbits in $\mathbf{M}_{\Omega} \cap\{i=0\}$ by Gabriel's theorem [Ga]. In particular, the irreducible components of $\Lambda$ are the closure of the conormal bundles of various $G_{\mathbf{v}}^{\mathbb{C}}$-orbits in $\mathbf{M}_{\Omega} \cap\{i=0\}$. Note that this gives another proof that $\Lambda$ is a Lagrangian subvariety of $\mathbf{M}$ for Dynkin graphs.

(4) For the graph of $A_{n}$ type and the very specific $V_{k}, W_{k}$, Goto introduced a certain Lagrangian subvariety in the quiver variety [Go]. His definition is totally different from ours, but the variety turns out to be the same under his situation. $\mathrm{He}$ also studied irreducible components of the Lagrangian subvariety. (The author thanks Dr. Goto for this remark.)

6. A stratification. We want to study the quiver variety $\mathfrak{M}_{\zeta}(\mathbf{v}, \mathbf{w})$ with nongeneric parameter $\zeta$ in this section. Then a point in $\mu^{-1}(\zeta)$ which has a nontrivial stabilizer corresponds to a singular point in $\mathfrak{M}_{\zeta}(\mathbf{v}, \mathbf{w})$.

We restrict our concern to the case when $\zeta$ has zero real component, i.e., $\zeta=$ $\left(0, \zeta_{\mathbb{C}}\right)$. (Many results in this section hold without this condition.) As in $\S 4$, take $\tilde{\zeta}=\left(\zeta_{\mathbb{R}}, \zeta_{\mathbb{C}}\right)$. We assume that $\left(\zeta_{\mathbb{R}}, 0\right)$ is generic, so $\tilde{\zeta}$ is also generic.

We introduce the following definition.

Definition 6.1 (Sjamaar-Lerman [SL]). For a subgroup $\hat{G}$ of $G_{v}$ denote by $\mathbf{M}_{(\hat{G})}$ the set of all points in $\mathbf{M}$ whose stabilizer is conjugate to $\hat{G}$. A point $[(B, i, j)] \in \mathfrak{M}_{\zeta}$ is said to be of $G$-orbit type $(\widehat{G})$ if its representative $(B, i, j)$ is in $\mathbf{M}_{(\hat{G})}$. The set of all points of orbit type $(\widehat{G})$ is denoted by $\left.(\mathfrak{M})_{\zeta}\right)_{(\widehat{G})}$.

This gives us a decomposition of $\mathfrak{M}_{\zeta}$,

$$
\mathfrak{M}_{\zeta}=\bigcup_{(\hat{G})}\left(\mathfrak{M}_{\zeta}\right)_{(\hat{G})},
$$


where the summation runs over the set of all conjugacy classes of subgroups of $G_{\mathrm{v}}$. Each stratum is a smooth hyper-Kähler manifold (cf. [SL]). The stratum $\left(\mathfrak{M}_{\zeta}\right)_{(1)}$ corresponding to the trivial subgroup 1 is $\mathfrak{M}_{\zeta}^{\text {reg }}$ by definition.

In $\S 4$ we defined the holomorphic map $\pi: \mathfrak{M}_{\tilde{\zeta}} \rightarrow \mathfrak{M}_{\zeta}$. We want to study a neighborhood of $\pi^{-1}(x)$ in $\mathfrak{M}_{\tilde{\zeta}}$ for a singular point $x \in \mathfrak{M}_{\zeta}$. The method is modeled on [Kr1, 3.3].

Let $m \in \mu^{-1}(-\zeta)$ be a representation of $x$ and let $\hat{G} \subset G_{\mathrm{v}}$ be the stabilizer of $m$. We decompose the Lie algebra $\mathfrak{g}_{\mathbf{v}}$ of $G_{\mathbf{v}}$ into linear subspaces $\hat{\mathfrak{g}} \oplus \hat{\mathfrak{g}}^{\perp}$ and write

$$
\mu(m+\varepsilon)+\zeta=\hat{\mu}(\varepsilon) \oplus v(\varepsilon) \in \mathbb{R}^{3} \otimes \hat{\mathfrak{g}}^{*} \oplus \mathbf{R}^{3} \otimes \hat{\mathfrak{g}}^{\perp *} .
$$

If $\mathbf{M}_{1} \subset T_{m} \mathbf{M}$ denotes the tangent space to the $G_{\mathbf{v}}$-orbit of $m$, every $G_{\mathbf{v}}$-orbit sufficiently close to $m$ intersects with $M_{1}^{\perp}$ in one orbit of $\hat{G}$. So there exists a homeomorphism

$$
\Phi: V \rightarrow U
$$

where $U$ (resp. $V$ ) is a neighborhood $x$ (resp. 0) in $\mathbf{M} / G_{\mathbf{v}}\left(\operatorname{resp} . \mathbf{M}_{1}^{\perp} / \hat{G}\right)$. We denote by $\mathscr{U} \subset \mathbf{M}$ (resp. $\mathscr{V} \subset \mathbf{M}_{1}^{\perp}$ ) the set of representatives of points in $U$ (resp. $V$ ).

Let $\hat{\mathbf{M}}$ be the orthogonal complement in $T_{m} \mathbf{M}$ to the $\mathbb{H}$-submodule $\mathbf{M}_{1} \oplus$ $I \mathbf{M}_{1} \oplus J \mathbf{M}_{1} \oplus K \mathbf{M}_{1}$. The space $\hat{\mathbf{M}}$ is itself an $\mathbf{I H}$-module, and the group $\hat{G}$ acts on it preserving this structure. The unique hyper-Kähler moment map on $\hat{\mathbf{M}}$ which vanishes at the origin is just the restriction of $\hat{\mu}$ to $\hat{\mathbf{M}}$; here $T_{m} \mathbf{M}$ is identified with $\mathbf{M}$.

As before, we have a holomorphic map $\hat{\pi}: \hat{\mu}^{-1}\left(-\tilde{\zeta}^{\prime}\right) / \hat{G} \rightarrow \hat{\mu}^{-1}(0) / \hat{G}$, where $\tilde{\zeta}^{\prime}=$ $\left(\zeta_{\mathbb{R}}, 0\right)$. Since we are assuming $\tilde{\zeta}^{\prime}$ is generic, $\hat{\mu}^{-1}\left(-\tilde{\zeta}^{\prime}\right) / \hat{G}$ is smooth. In this setting, we have the following.

Proposition 6.2. If $\zeta_{\mathbb{R}}$ is sufficiently small, a neighborhood of $\pi^{-1}(x)$ in $\mathfrak{M}_{\tilde{\zeta}}$ is diffeomorphic to a neighborhood of $\hat{\pi}^{-1}(0)$ in $\hat{\mathbf{M}} \cap \hat{\mu}^{-1}\left(-\tilde{\zeta}^{\prime}\right) / \hat{G}$.

Proof. We prove Proposition 6.2 by replacing $\zeta_{\mathbb{R}}$ with $\lambda^{2} \zeta_{\mathbb{R}}$ with sufficiently small $\lambda$. The key to the proof is the homogeneity of the moment map. The dilatation on $T_{m} \mathbf{M}$ transforms $\hat{\mu}$ and $v$ as

$$
\begin{aligned}
& \hat{\mu}(\lambda \varepsilon)=\lambda^{2} \hat{\mu}(\varepsilon) \\
& v(\lambda \varepsilon)=\lambda d v_{0}(\varepsilon)+\lambda^{2} \mu^{\perp}(\varepsilon),
\end{aligned}
$$

where $d v_{0}$ is the derivative of $v$ at 0 , and $\mu^{\perp}(\varepsilon)$ is the $\hat{g}^{\perp}$-component of $\mu(\varepsilon)$. (We identify $T_{m} \mathbf{M}$ with $\mathbf{M}$.) Hence $m+\lambda \varepsilon \in \mu^{-1}\left(-\left(\lambda^{2} \zeta_{\mathbb{R}}, \zeta_{\mathbb{C}}\right)\right)$ if and only if

$$
\varepsilon \in \hat{\mu}^{-1}\left(-\tilde{\zeta}^{\prime}\right), d v_{0}(\varepsilon)+\lambda \mu^{\perp}(\varepsilon)=-\lambda \tilde{\zeta}^{\prime} .
$$

As $\lambda \rightarrow 0, \mathbf{M}_{1}^{\perp} \cap \mu^{-1}\left(-\left(\lambda^{2} \zeta_{\mathbb{R}}, \zeta_{\mathbb{C}}\right)\right)$ converges to $\mathbf{M}_{1}^{\perp} \cap \operatorname{Ker} d v_{0} \cap \hat{\mu}^{-1}\left(-\tilde{\zeta}^{\prime}\right)=\hat{\mathbf{M}} \cap$ 
$\hat{\mu}^{-1}\left(-\tilde{\zeta}^{\prime}\right)$. In particular, if we denote the ball of radius $R$ by $B_{R}$, there exists a $\widehat{G}$-equivariant diffeomorphism between $B_{\lambda R} \cap \mathbf{M}_{1}^{\perp} \cap \mu^{-1}\left(-\left(\lambda^{2} \zeta_{\mathbb{R}}, \zeta_{\mathbb{C}}\right)\right)$ and $B_{R} \cap$ $\hat{\mathbf{M}} \cap \hat{\mu}^{-1}\left(-\tilde{\zeta}^{\prime}\right)$. If $\lambda$ is sufficiently small, $B_{\lambda R}$ is contained in $\mathscr{V}$. Thus the only remaining thing to prove is that $\hat{\pi}^{-1}(0) \subset B_{R} / \hat{G}$ and $\pi^{-1}(x) \subset \Phi\left(B_{\lambda R} / \widehat{G}\right)$.

Take $y \in \pi^{-1}(x) \subset \mathfrak{M}_{\left(\lambda^{2} \zeta_{\mathbb{R}}, \zeta_{\mathbb{C}}\right)}$ and let $m^{\prime}$ be its representative. The condition $\pi(y)=x$ is equivalent to saying that $G_{v}^{\mathbb{C}} m$ is the unique closed $G_{v}^{\mathbb{C}}$-orbit contained in the closure of $G_{v}^{\mathbb{C}} m^{\prime}$. By [Nee], [Sc], the path of steepest descent for $\left\|\mu_{\mathbb{R}}\right\|^{2}$ from $m^{\prime}$ converges to a point contained in the orbit $G_{v} m$. The corresponding also holds for $\hat{\pi}$. Hence we can take $R$ so that the representatives of points in $\hat{\pi}^{-1}(0) \subset$ $\hat{\mu}^{-1}\left(-\tilde{\zeta}^{\prime}\right)$ are contained in the ball $B_{R}$. We then show that, if $\lambda$ is sufficiently small, we have

$$
\operatorname{dist}\left(m^{\prime}, G_{\mathbf{v}} m\right)<\lambda R \quad \text { for } G_{\mathbf{v}} m^{\prime} \in \pi^{-1}(x) \subset \mathfrak{M}_{\left(\lambda^{2} \zeta_{\mathbb{R}}, \zeta_{\mathbb{C}}\right.} .
$$

Suppose the contrary. There exists a sequence $\lambda_{i}$ converging to 0 and $m_{i}^{\prime}$ such that $\operatorname{dist}\left(m_{i}^{\prime}, G_{\mathrm{v}} m\right) \geqslant \lambda_{i} R$. We may assume that the limit of the path of steepest decent for $\left\|\mu_{\mathbb{R}}\right\|^{2}$ from $m_{i}^{\prime}$ is $m$. By assumption, the path of steepest decent must intersect with the sphere $\partial B_{\lambda_{i} R}(m)$ of radius $\lambda_{i} R$ centered at $m$. Let $p_{i}$ be a point in the intersection. As above, we use the dilatation and write $p_{i}=m+\lambda_{i} \varepsilon_{i}$. We have

$$
\left\|\varepsilon_{i}\right\|=R,
$$

$$
\lambda_{i}^{2}\left\|\hat{\mu}_{\mathbb{R}}\left(\varepsilon_{i}\right)\right\|^{2}+\left\|d v_{0}^{\mathbb{R}}(\varepsilon)+\lambda_{i} \mu_{\mathbb{R}}^{\perp}(\varepsilon)\right\|^{2}=\lambda_{i}^{-2}\left\|\mu_{\mathbb{R}}\left(p_{i}\right)\right\|^{2} \leqslant \lambda_{i}^{-2} \mu_{\mathbb{R}}\left(m_{i}^{\prime}\right) \|^{2}=\lambda_{i}^{2},
$$

where $d v_{0}^{\mathbb{R}}$ (resp. $\mu_{\mathbb{R}}^{\perp}$ ) is the $\mathbb{R}$-component of $d v_{0}$ (resp. $\mu^{\perp}$ ). We may assume that $\varepsilon_{i}$ converges to $\varepsilon_{\infty}$ as $i \rightarrow \infty$. We have $\left\|\varepsilon_{\infty}\right\|=R, d v_{0}^{\mathbb{R}}\left(\varepsilon_{\infty}\right)=0$ from (6.4). On the other hand, the path of steepest descent for $\left\|\mu_{\mathbb{R}}\right\|^{2}$ converges to that of $\left\|d v_{0}^{\mathbb{R}}\right\|^{2}$, and the path from $\varepsilon_{\infty}$ goes to 0 . This is a contradiction. Thus we have (6.3).

We now give concrete descriptions of the stratum $\left(\mathfrak{M}_{\zeta}\right)_{(\hat{G})}$ and $\hat{\mathbf{M}} \cap \hat{\mu}^{-1}\left(-\tilde{\zeta}^{\prime}\right) / \widehat{G}$. Let us define $R_{+}(\mathbf{v} ; \zeta)$ by

$$
R_{+}(\mathbf{v} ; \zeta) \stackrel{\text { def. }}{=}\left\{\theta \in R_{+}(\mathbf{v}) \mid \zeta \in \mathbb{R}^{3} \otimes D_{\theta}\right\} .
$$

Lemia 6.5. Let $x \in\left(\mathfrak{M}_{\zeta}\right)_{(\hat{G})}$ with a nontrivial $\hat{G}$. Then there is an orthogonal decomposition

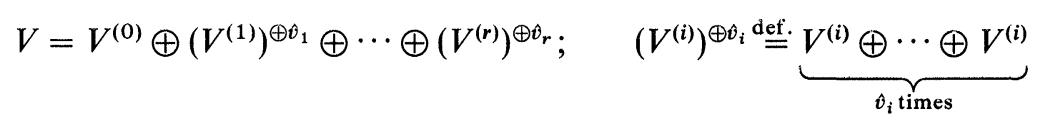

(as collections of vector spaces indexed by the vertices of the graph) such that a representative $(B, i, j)$ of $x$ has the following properties:

(1) each summand is invariant under $B$;

(2) the restriction of $B$ to $\left(V^{(i)}\right)^{\oplus \theta_{i}}=\mathbb{C}^{\hat{\theta}_{i}} \otimes V^{(i)}$ is of the form $\left.1_{\mathbb{C}^{v_{i}}} \otimes B\right|_{V^{(i)}}$; 
(3) the image of $i$ is contained in $V^{(0)}$, and $j$ is zero on $V \ominus V^{(0)}$;

(4) for each $V^{(i)}$ with $i \geqslant 1$, its dimension vector $\mathbf{v}^{(i)} \stackrel{\operatorname{def} . t}{=}\left(\operatorname{dim} V_{1}^{(i)}, \ldots, \operatorname{dim} V_{n}^{(i)}\right)$ belongs to $R_{+}(\mathbf{v} ; \zeta)$;

(5) the restriction of $(B, i, j)$ to $V^{(0)}$ has the trivial stabilizer in $G_{\mathrm{v}^{(0)}}=\prod_{k} \mathrm{U}\left(V_{k}^{(0)}\right)$ (though the case $V^{(0)}=0$ is not excluded);

(6) the subgroup $G_{\mathbf{v}^{(i)}}=\prod_{k} \mathrm{U}\left(V_{k}^{(i)}\right)$ meets the stabilizer $\hat{G}$ only in the scalar subgroup:

$$
G_{\mathbf{v}^{(i)}} \cap \hat{G} \cong \mathrm{U}(1) \subset G_{\mathbf{v}^{(i)}}
$$

(7) if $i \neq j$, there is no isometry $V^{(i)} \rightarrow V^{(j)}$ which commutes with $B$.

Proof. Let $(B, i, j)$ be a representative of $x$. The proof of $(2.8)$ shows that there exists a decomposition

$$
V=V^{0} \oplus V^{\prime} \oplus V^{\prime \prime} \oplus \cdots
$$

with the following properties:

(a) each summand is invariant under $B$;

(b) the image of $i$ is contained in $V^{0}$ and $j$ is zero on $V^{\prime} \oplus V^{\prime \prime} \oplus \cdots$;

(c) the restriction of $B$ to $V^{\prime \prime \cdots}$ satisfies conditions (4) and (6) by replacing $V^{(i)}$ with $V^{\prime \prime \cdots \prime}$.

Define an equivalence relation on the summands $V^{\prime}$, etc., by declaring $V^{\prime} \sim V^{\prime \prime}$ if and only if there exists an isometry $V^{\prime} \rightarrow V^{\prime \prime}$ which commutes with $B$. Collecting equivalence classes of summands, we have the desired decomposition.

Thus we have the description of the stratum

$$
\left(\mathfrak{M}_{\zeta}\right)_{(\hat{G})} \cong \mathfrak{M}_{\zeta}^{\text {reg }}\left(\mathbf{v}^{(0)}, \mathbf{w}\right) \times \mathfrak{M}_{\zeta}^{\mathrm{reg}}\left(\hat{v}_{1}, \mathbf{v}^{(1)} ; \ldots ; \hat{v}_{r}, \mathbf{v}^{(r)}\right)
$$

where

$$
\mathfrak{M}_{\zeta}^{\text {reg }}\left(\hat{v}_{1}, \mathbf{v}^{(1)} ; \ldots ; \hat{v}_{r}, \mathbf{v}^{(r)}\right) \stackrel{\text { def. }}{=}\{B \in \mathbf{M} \mid B \text { as in }(6.5)(1),(2),(6),(7)\} / \prod_{i=1}^{r} G_{\mathbf{v}^{(i)}}
$$

The situation is simple when the graph is of Dynkin type and $\zeta=0$.

Proposition 6.7. Assume that our graph is of type $A, D, E$. Then $\mathfrak{M}_{0}(\mathbf{v}, 0)=$ $\{0\}$ for any v. Hence each stratum $\left(\mathfrak{M}_{0}\right)_{(\hat{G})}$ is isomorphic to $\mathfrak{M}_{0}^{\mathrm{reg}}\left(\mathbf{v}^{(0)}, \mathbf{w}\right)$, and we have a decomposition

$$
\bigcup \mathfrak{M}_{0}^{\mathrm{reg}}\left(\mathbf{v}^{(0)}, \mathbf{w}\right) \cong \mathfrak{M}_{0}(\mathbf{v}, \mathbf{w})
$$

where $\mathbf{v}^{(0)}$ runs over the set of dimensions of subspaces $V^{(0)}$ of $V$ as in (6.2). 
Proof. Take $B \in \mu^{-1}(0) \subset \mathbf{M}(\mathbf{v}, 0)$. We want to show tht $B=0$. As in the proof of (2.8), we may assume that $G_{\mathrm{v}} / \mathrm{U}(1)$ acts freely on the $B$-orbit. Then the dimension formula (2.11) implies

$$
\operatorname{dim}_{\mathbb{C}} \mu^{-1}(0) /\left(G_{\mathbf{v}} / \mathrm{U}(1)\right)=2-{ }^{t} \mathbf{v} \mathbf{V}=0,
$$

since the Cartan matrix is positive definite. Hence $\mu^{-1}(0) /\left(G_{\mathrm{v}} / \mathrm{U}(1)\right)$ consists of discrete points. In particular, the $G_{\mathrm{v}}$-orbit [B], considered as a point in $\mu^{-1}(0) /$ $\left(G_{\mathrm{v}} / \mathrm{U}(1)\right)$, is a fixed point of the $\mathbb{C}^{*}$-action defined as in $\S 5$. Hence (5.3) (2) shows that $B=0$.

We return to the general case. Suppose that $x$ is in the stratum (6.6). Let $m$ be its representative. Then $V$ decomposes as in (6.5); hence $\hat{\mathbf{M}}$ decomposes into

$$
\begin{aligned}
& \hat{\mathbf{M}} \cong\left(\hat{\mathbf{M}} \cap \mathbf{M}\left(\mathbf{v}^{(0)}, \mathbf{w}\right)\right) \oplus\left(\bigoplus_{i, j=1}^{r} \hat{M}_{i j} \otimes \operatorname{Hom}\left(\mathbb{C}^{\hat{\theta}_{i}}, \mathbb{C}^{\hat{\theta}_{j}}\right)\right) \\
& \oplus\left(\bigoplus_{i=1}^{r} \operatorname{Hom}\left(\mathbb{C}^{\hat{\theta}_{i}}, \hat{W}_{i}\right)\right) \oplus\left(\bigoplus_{i=1}^{r} \operatorname{Hom}\left(\hat{W}_{i}, \mathbb{C}^{\hat{\theta}_{i}}\right)\right)
\end{aligned}
$$

where

$$
\begin{aligned}
& \hat{M}_{i j} \stackrel{\text { def. }}{=} \hat{\mathbf{M}} \cap\left(\bigoplus_{h \in H} \operatorname{Hom}\left(V_{\mathrm{out}(h)}^{(i)}, V_{\mathrm{in}(h)}^{(j)}\right)\right) \\
& \hat{W}_{i} \stackrel{\text { def. }}{=} \hat{\mathbf{M}} \cap\left\{\left(\bigoplus_{h \in H} \operatorname{Hom}\left(V_{\mathrm{out}(h)}^{(0)}, V_{\mathrm{in}(h)}^{(i)}\right)\right) \oplus\left(\bigoplus_{k=1}^{n} \operatorname{Hom}\left(W_{k}, V_{k}^{(i)}\right)\right)\right\} .
\end{aligned}
$$

The stabilizer $\hat{G}$ is given by $\prod_{i} \mathrm{U}\left(\hat{v}_{i}\right)$, and acts trivially on the component

$$
T \stackrel{\text { def. }}{=}\left(\hat{\mathbf{M}} \cap \mathbf{M}\left(\mathbf{v}^{(0)}, \mathbf{w}\right)\right) \oplus\left(\bigoplus_{i=1}^{r} \hat{M}_{i i} \otimes 1_{\mathbb{C}^{\hat{t}_{i}}}\right) .
$$

By using the conditions (6.5) (5), (6), we can check that the component $T$ can be identified with the tangent space $T_{x}\left(\mathfrak{M}_{\zeta}\right)_{(\hat{G})}$ of the stratum containing $x$.

Let $T^{\perp}$ be the orthogonal complement in $\hat{\mathbf{M}}$. Then we have the factorization

$$
\hat{\mathbf{M}} \cap \hat{\mu}^{-1}\left(-\tilde{\zeta}^{\prime}\right) / \hat{G}=T \times\left(T^{\perp} \cap \hat{\mu}^{-1}\left(-\tilde{\zeta}^{\prime}\right)\right) / \hat{G} .
$$

Define a symmetric $(r \times r)$-matrix $\hat{\mathbf{A}}$ by setting its $(i, j)$-component to be $\operatorname{dim}_{\mathbb{C}} \hat{M}_{i j}$, The second component $\mathcal{S}^{\text {def. }}=\left(T^{\perp} \cap \hat{\mu}^{-1}\left(-\tilde{\zeta}^{\prime}\right)\right) / \hat{G}$ is also a quiver variety on a graph whose adjacency matrix of the graph is given by $\hat{\mathbf{A}}$, where we allow edge loops. Its dimension data are given by

$$
\mathbf{v}_{s}={ }^{t}\left(\hat{v}_{1}, \hat{v}_{2}, \ldots, \hat{v}_{r}\right), \quad \mathbf{w}_{s}={ }^{t}\left(\operatorname{dim}_{\mathbb{C}} \hat{W}_{1}, \operatorname{dim}_{\mathbb{C}} \hat{W}_{2}, \ldots, \operatorname{dim}_{\mathbb{C}} \hat{W}_{r}\right) .
$$


Note that

(6.9) $\operatorname{dim}_{\mathbb{R}} \mathfrak{S}=\operatorname{dim}_{\mathbb{R}} \hat{\mathbf{M}} \cap \hat{\mu}^{-1}\left(-\tilde{\zeta}^{\prime}\right) / \hat{G}-\operatorname{dim}_{\mathbb{R}} T=\operatorname{dim}_{\mathbb{R}} \mathfrak{M}_{\zeta}^{\mathrm{reg}}-\operatorname{dim}_{\mathbb{R}}\left(\mathfrak{M}_{\zeta}\right)_{(\hat{G})}$.

Under the same assumption as (6.7), $V^{(i)}$ has the 1-dimensional vector space on the vertex $i$ and 0 and the other vertices. The matrix $\left(\hat{a}_{i j}\right)$ is equal to the original adjacency matrix. We then have simple formulas for $\mathbf{v}_{s}, \mathbf{w}_{s}$ :

$$
\mathbf{v}_{s}=\mathbf{v}-\mathbf{v}^{(0)}, \quad \mathbf{w}_{s}=\mathbf{w}-\mathbf{C v}^{(0)} .
$$

Let $\mathfrak{M}_{\left(\lambda^{2} \zeta_{\mathbb{R}}, \zeta_{\mathbb{C}}\right)} \cong H_{\left(\lambda^{2} \zeta_{\mathbb{R}}, \zeta_{\mathbb{C}}\right)}^{\mathrm{s}} / G_{\mathbf{v}}^{\mathbb{C}}$ be the holomorphic description in $\S 3$. Exactly as in the proof of (5.1), one can prove that there exists a $\mathbb{C}^{*}$-action which is an extension of the $S^{1}$-action given by $[(B, i, j)] \mapsto[(t B, t i, t j)]$. It implies that $H_{\left(\zeta_{\mathbb{R}}, \zeta_{\mathbb{C}}\right)}^{\mathrm{s}}$ corresponds bijectively to $H_{\left(t^{2} \zeta_{\mathbb{R}}, t^{2} \zeta_{\mathbb{G}}\right)}^{\mathrm{s}}$ under $(B, i, j) \mapsto(t B, t i, t j)$. In particular, $H_{\left(\lambda^{2} \zeta_{\mathbb{R}}, \zeta_{\mathbb{C}}\right)}^{\mathrm{s}}$ is independent of $\lambda$. So we have an into diffeomorphism

$$
F_{\lambda}: \text { a neighborhood of } \hat{\pi}^{-1}(0) \text { in } \hat{\mathbf{M}} \cap \hat{\mu}^{-1}\left(-\lambda^{2} \tilde{\zeta}^{\prime}\right) / \hat{G} \rightarrow H_{\tilde{\zeta}}^{\mathbf{s}} / G_{\mathbf{v}}^{\mathbb{C}}
$$

for small $\lambda$. We can take the neighborhood so that its cohomology group is isomorphic to $H^{*}\left(\hat{\pi}^{-1}(0) ; \mathbb{Z}\right)$. By $(5.4)$ this is isomorphic to $H^{*}(\Im ; \mathbb{Z})$ (see (6.8)). The above proof shows the image of $F_{\lambda}$ converges to $\pi^{-1}(x)$ as $\lambda \rightarrow 0$. Hence the continuity of the cohomology implies the following.

THEOREM 6.10. We have an isomorphism between the cohomology groups,

$$
H^{*}\left(\pi^{-1}(x) ; \mathbb{Z}\right) \cong H^{*}(\Im ; \mathbb{Z})
$$

Combining (6.9), (6.10), and the fact that $H^{d}(\mathfrak{S} ; \mathbb{Z})=0$ if $d>\operatorname{dim}_{\mathbb{C}} \mathfrak{S}$ ( $\mathfrak{S}$ is diffeomorphic to an affine algebraic manifold), we get the following.

COROLlaRy 6.11. With respect to the stratification (6.3), the map $\pi: \mathfrak{M}_{\xi} \rightarrow \mathfrak{M}_{\zeta}$ is semismall in the sense of [BM,1.1], that is,

$$
2 \operatorname{dim}_{\mathbb{R}} \pi^{-1}(x) \leqslant \operatorname{dim}_{\mathbb{R}} \mathfrak{M}_{\zeta}^{\text {reg }}-\operatorname{dim}_{\mathbb{R}}\left(\mathfrak{M}_{\zeta}\right)_{(\hat{G})}
$$

for $x \in\left(\mathfrak{M}_{\zeta}\right)_{(\hat{G})}$.

As we promised before, we now finish the proof of Theorem 4.1.

Proof of Theorem 4.1. When $\mathfrak{M}_{\zeta}^{\text {reg }} \neq \varnothing$, it is the set of nonsingular points in $\mathfrak{M}_{\zeta}$. The only thing remaining to show is that $\pi^{-1}\left(\mathfrak{M}_{\zeta}^{\text {reg }}\right)$ is dense in $\mathfrak{M}_{\tilde{\zeta}}$. It may possibly happen that $\mathfrak{M}_{\tilde{\zeta}}$ has a component $\mathscr{C}$ which is mapped to the singular points in $\mathfrak{M}_{\zeta}$ by $\pi$.

Suppose that the component $\mathscr{C}$ is mapped to the closure of a stratum $\left(\mathfrak{M}_{\zeta}\right)_{(\hat{G})}$. If the image of $\mathscr{C}$ is contained in $\overline{\left(\mathfrak{M}_{\zeta}\right)_{(\widehat{G})}} \backslash\left(\mathfrak{M}_{\zeta}\right)_{(\hat{G})}$, we go to a lower stratum. We continue this procedure until either of the following is satisfied: (a) $\mathscr{C}$ is mapped 
to a point, or (b) $\mathscr{C}$ is mapped to the closure of a stratum $\left(\mathfrak{M}_{\zeta}\right)_{(\hat{G})}$ and the image intersects with $\left(\mathfrak{M}_{\zeta}\right)_{(\hat{G})}$. In the case (a), $\mathscr{C}$ is a compact component. This is a contradiction since $\mathfrak{M}_{\tilde{\zeta}}$ is diffeomorphic to an affine algebraic manifold (Remark 3.3 (3) and Corollary 4.2). In the case (b), we take a point $x$ in the intersection. Then a compact subvariety $\pi^{-1}(x) \cap \mathscr{C}$ satisfies

$$
\operatorname{dim}_{\mathbb{R}}\left(\pi^{-1}(x) \cap \mathscr{C}\right) \geqslant \operatorname{dim}_{\mathbb{R}} \mathscr{C}-\operatorname{dim}_{\mathbb{R}}\left(\mathfrak{M}_{\zeta}\right)_{(\hat{G})}=\operatorname{dim}_{\mathbb{R}} \mathfrak{S}
$$

Since homology groups of $\mathfrak{S}$ vanish in degree greater than $(1 / 2) \operatorname{dim}_{\mathbb{R}} \mathfrak{S}$, the fundamental class of $\pi^{-1}(x) \cap \mathscr{C}$ vanishes by Theorem 6.10. This is a contradiction.

7. Examples of quiver varieties I: Cotangent bundles of generalized flag manifolds of type $\boldsymbol{A}_{\boldsymbol{n}}$. The purpose of this and the next sections is to give concrete examples of quiver varieties. Surprisingly, some nilpotent varieties and related varieties appear though they seem to have no relation to quivers at first sight. In this section we observe that cotangent bundles of generalized flag manifolds of type $A_{n}$ arise as $\mathfrak{M}_{\zeta}(\mathbf{v}, \mathbf{w})$ for specific data $\mathbf{v}, \mathbf{w}$.

For a strictly decreasing sequence of integers $r>v_{1}>v_{2}>\cdots>v_{n}>0$, we denote by $\mathscr{F}=\mathscr{F}\left(v_{1}, \ldots, v_{n} ; r\right)$ the manifold consisting of all sequences $\phi=\left(\mathbb{C}^{r}=\right.$ $\left.E^{0} \supset E^{1} \supset \cdots \supset E^{n} \supset E^{n+1}=0\right)$ of subspaces of $\mathbb{C}^{r}$ with $\operatorname{dim} E^{k}=v_{k}(1 \leqslant k \leqslant n)$. This is a generalized flag manifold of type $A_{n}$. Its cotangent bundle $T^{*} \mathscr{F}$ is described as

$$
\left\{(\phi, A) \in \mathscr{F} \times \operatorname{End}\left(\mathbb{C}^{r}\right) \mid A\left(E^{k}\right) \subset E^{k+1} \quad \text { for } k=0,1, \ldots, n\right\}
$$

Let $\mathbf{v}={ }^{t}\left(v_{1}, v_{2}, \ldots, v_{n}\right), \mathbf{w}={ }^{t}(r, 0, \ldots, 0)$ and consider $\mathfrak{M}_{\zeta}=\mathfrak{M}_{\zeta}(\mathbf{v}, \mathbf{w})$; here the orientation of the Dynkin graph of type $A_{n}$ is as follows:

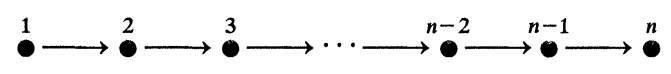

Then the following was shown by Kraft and Procesi [KP] (see also Remark 8.5 (3)).

THEOREM 7.2. The map

$$
\mathfrak{M}_{0} \ni[(B, i, j)] \mapsto j_{1} i_{1} \in \operatorname{End}\left(\mathbb{C}^{r}\right)
$$

is an isomorphicm onto the closure of the conjugacy class of a nilpotent matrix.

In fact, they proved the above for the affine algebraic quotient $\mu_{\mathbb{C}}^{-1}(0) / / G_{\mathbf{v}}^{\mathbb{C}}$. So our assertion follows from Theorem 3.1.

THEOREM 7.3. Under the assumption (3.4), $\mathfrak{M}_{\left(\zeta_{\mathbb{R}}, 0\right)}$ is isomorphic to $T^{*} \mathscr{F}$ as a complex manifold. 
Proof. We identify the set $H$ of oriented edges with $\{(k, l) \mid 1 \leqslant k, l \leqslant n$, $|k-l|=1\}$. We denote $B_{h}$ by $B_{k, l}$ where $k=\operatorname{in}(h), l=\operatorname{out}(h)$. This is the notation adapted in [KN].

We first show that $B_{k-1, k}(k=2,3, \ldots, n)$ and $j_{1}$ are injective by induction. The real ADHM equation

$$
B_{n, n-1} B_{n, n-1}^{\dagger}-B_{n-1, n}^{\dagger} B_{n-1, n}=-\zeta_{\mathbb{R}}^{(n)} 1_{V_{n}}
$$

and $\zeta_{\mathbb{R}}^{(n)}>0$ imply $B_{n-1, n}$ is injective.

If $B_{k-1, k}$ is injective, the complex ADHM equation

$$
B_{k-1, k} B_{k, k-1}-B_{k-1, k-2} B_{k-2, k-1}=0
$$

shows ker $B_{k-2, k-1} \subset \operatorname{ker} B_{k, k-1}$. Then the real ADHM equation

$$
\begin{aligned}
& B_{k-1, k} B_{k-1, k}^{\dagger}-B_{k, k-1}^{\dagger} B_{k, k-1}+B_{k-2, k-1} B_{k-1, k-2}^{\dagger}-B_{k-1, k-2}^{\dagger} B_{k-2, k-1} \\
& =-\zeta_{\mathbb{R}}^{(k-1)} 1_{V_{k-1}}
\end{aligned}
$$

and $\zeta_{\mathbb{R}}^{(k-1)}>0$ imply that $B_{k-2, k-1}$ is injective. The proof for the final step (i.e., $j_{1}$ is injective) is the same. The above shows that there exists a natural map

$$
\mathfrak{M}_{\zeta} \rightarrow H_{\zeta}^{\mathrm{s}} / G_{\mathbf{v}} \stackrel{\mathbb{C} \text { def. }}{=}\left\{(B, i, j) \mid \mu_{\mathbb{C}}(B, i, j)=0, B_{k-1, k} \text { and } j_{1} \text { are injective }\right\} / G_{\mathbf{v}}^{\mathbb{C}},
$$

where $\zeta=\left(\zeta_{\mathbb{R}}, 0\right)$. By the general properties of Kähler quotients (see [Ki, Lemma 7.2]), this map is injective. (The notation $H_{\zeta}^{\mathrm{s}}$ was used for a different meaning in $\S 3$, but the following discussion shows that it turns out to be the same.)

We next show that $H_{\zeta}^{\mathrm{s}} / G_{\mathbf{v}}^{\mathbb{C}}$ is isomorphic to $T^{*} \mathscr{F}$. For $(B, i, j) \in H_{\zeta}^{\mathrm{s}}$, consider a sequence of subspaces $\mathbb{C}^{r}=E^{0} \supset E^{1} \supset E^{2} \supset \cdots \supset E^{n} \supset E^{n+1}=0$, where $E^{k}=$ $\operatorname{Im} j_{1} B_{1,2} B_{2,3} \cdots B_{k-1, k}(1 \leqslant k \leqslant n)$. This induces a map $p: H_{\zeta}^{\mathrm{s}} / G_{\mathrm{v}}^{\mathbb{C}} \rightarrow F$. We also define a map $\pi: H_{\zeta}^{\mathrm{s}} / G_{\mathrm{v}}^{\mathbb{C}} \rightarrow \operatorname{End}\left(\mathbb{C}^{r}\right)$ by $\pi([(B, i, j)])=j_{1} i_{1}$. The complex ADHM equation implies the image $(p \times \pi)\left(H_{\zeta}^{\mathrm{s}} / G_{\mathbf{v}}^{\mathbb{C}}\right) \subset \mathscr{F} \times \operatorname{End}\left(\mathbb{C}^{r}\right)$ is contained in $T^{*} \mathscr{F}$. The map $p \times \pi$ is holomorphic.

Suppose that $[(B, i, j)],\left[\left(B^{\prime}, i^{\prime}, j^{\prime}\right)\right] \in H_{\zeta}^{\mathrm{s}} / G_{\mathbf{v}}^{\mathbb{C}}$ have the same image under the map $p \times \pi$. Since $p([(B, i, j)])=p\left(\left[\left(B^{\prime}, i^{\prime}, j^{\prime}\right)\right]\right)$, we may assume that

$$
j_{1}=j_{1}^{\prime}, \quad B_{1,2}=B_{1,2}^{\prime}, \ldots, B_{n-1, n}=B_{n-1, n}^{\prime}
$$

by replacing $\left(B^{\prime}, i^{\prime}, j^{\prime}\right)$ by $g .\left(B^{\prime}, i^{\prime}, j^{\prime}\right)$. Then $\pi([(B, i, j)])=\pi\left(\left[\left(B^{\prime}, i^{\prime}, j^{\prime}\right)\right]\right)$ and the injectivity of $j_{1}$ imply $i_{1}=i_{1}^{\prime}$. Then the complex ADHM equation $B_{1,2} B_{2,1}+$ $i_{1} j_{1}=0=B_{1,2} B_{2,1}^{\prime}+i_{1} j_{1}$ and the injectivity of $B_{1,2}$ show $B_{2,1}=B_{2,1}^{\prime}$. We repeat the argument inductively to get $B_{k, k-1}=B_{k, k-1}^{\prime}$. This shows that $p \times \pi: H_{\zeta}^{\mathrm{s}} / G_{\mathrm{v}}^{\mathbb{C}} \rightarrow$ $T^{*} \mathscr{F}$ is injective. It is also easy to check the surjectivity.

We finally show that the composition $\mathfrak{M l}_{\zeta} \rightarrow T^{*} \mathscr{F}$ is an isomorphism. The image 
of $\mathfrak{M}_{\zeta}$ is open in $H_{\zeta}^{\mathrm{s}} / G_{\mathbf{v}}^{\mathbb{C}}$ (The solvability of the real moment map equation is an open condition, when the stabilizer is trivial.) Since $T^{*} \mathscr{F}$ is connected, it is enough to show that the image is closed. But this is contained in Theorem 7.2: the map $\mathfrak{M}_{0} \ni[(B, i, j)] \mapsto j_{1} i_{1} \in \operatorname{End}\left(\mathbb{C}^{r}\right)$ is proper.

8. Examples of quiver varieties II: Nilpotent varieties of type $A_{n}$ and their intersections with transversal slices. In this section we assume that the graph is of type $A_{n}$. We observe that quiver varieties $\mathfrak{M}_{\zeta}$ relate to known varieties, which are studied extensively in conjunction with representations of Weyl groups (see, e.g., [BM]).

We first begin with a simple lemma, which holds for any graph. Let $\mathbf{u}=$ $\mathbf{w}-\mathbf{C v} \in \mathbb{Z}^{n}$.

LEMMA 8.1. If $\mathfrak{M}_{0}^{\mathrm{reg}} \neq \varnothing$, then components of $\mathbf{u}$ are nonnegative.

Proof. Let $[(B, i, j)] \in \mathfrak{M}_{0}^{\text {reg }}$. Fix a vertex $k$ and consider the operator

$$
\mathscr{D} \stackrel{\text { def. }}{=}\left(\begin{array}{cc}
B_{\bar{h}} & \varepsilon(h) B_{h}^{\dagger} \\
j_{k} & i_{k}^{\dagger}
\end{array}\right): \mathbb{C}^{2} \otimes V_{k} \rightarrow\left(\bigoplus_{h \in H: k=\text { in }(h)} V_{\text {out }(h)}\right) \oplus W_{k}
$$

The ADHM equation implies

$$
\mathscr{D}^{\dagger} \mathscr{D}=\left(\begin{array}{cc}
\Delta & 0 \\
0 & \Delta
\end{array}\right): \mathbb{C}^{2} \otimes V_{k} \rightarrow \mathbb{C}^{2} \otimes V_{k}
$$

for some nonnegative operator $\Delta: V_{k} \rightarrow V_{k}$.

If $\Delta$ has a kernel, $(B, i, j)$ has a nontrivial stabilizer in $G_{\mathbf{v}}$. This is a contradiction. Hence $\Delta$ is an isomorphism, and $\mathscr{D}$ is injective. So we have

$$
2 \operatorname{dim} V_{k} \leqslant \sum_{h \in H: k=\operatorname{in}(h)} \operatorname{dim} V_{\text {out }(h)}+\operatorname{dim} W_{k} .
$$

This proves the assertion.

We now restrict our concern to type $A_{n}$, and number the vertices as in $\S 7$. Let $\lambda$ be a partition of $r=\sum_{k} k w_{k}$ (i.e., $\lambda=\left(\lambda_{1}, \lambda_{2}, \ldots, \lambda_{N}\right)$ with $\lambda_{i} \in \mathbb{N}, \sum \lambda_{i}=r$ and $\lambda_{1} \geqslant \lambda_{2} \geqslant \cdots \geqslant \lambda_{N}$ ) by

$$
\lambda=(\underbrace{n, n, \ldots, n}_{w_{n} \text { times }}, \ldots, \underbrace{k, k, \ldots, k}_{w_{k} \text { times }}, \ldots, \underbrace{1,1, \ldots, 1)}_{w_{1} \text { times }} .
$$

We also define a sequence $\mu=\left(\mu_{1}, \mu_{2}, \ldots, \mu_{n+1}\right)$ by

$$
\mu_{i}=v_{n}+\sum_{k \geqslant i} u_{k} \quad \text { for } i=1,2, \ldots, n, \quad \mu_{n+1}=v_{n} .
$$


By Lemma 8.1 this is a nonincreasing sequence, and we consider it as a partition of $r$ (same as $\lambda$ ) by removing 0 in the sequence. Let $N_{\lambda}$ (resp. $N_{\mu}$ ) be an $r \times r$ nilpotent matrix with Jordan cells of size $\lambda_{1}, \lambda_{2}, \ldots, \lambda_{N}\left(\operatorname{resp} . \mu_{1}, \mu_{2}, \ldots, \mu_{n+1}\right)$, and let $\mathcal{O}_{\lambda}$ (resp. $\mathcal{O}_{\mu}$ ) be its conjugacy class. Let $\mathscr{S}_{\lambda}$ denote the transversal slice to $\mathcal{O}_{\lambda}$ at $N_{\lambda}$ constructed by Slodowy [S2]. It has the following property:

$\mathscr{S}_{\lambda}$ meets only those conjugacy classes whose closures contain $\mathcal{O}_{\lambda}$, and its intersection with those orbits is transverse.

Then the following is a reformulation of a result of Kronheimer [Kr2].

THEOREM 8.4. There is a natural biholomorphic map

$$
\mathfrak{M}_{0}^{\mathrm{reg}} \cong \mathcal{O}_{\mu} \cap \mathscr{S}_{\lambda}
$$

Proof. We regard $\mathfrak{M}_{0}^{\text {reg }}$ as a moduli space of $\mathrm{SU}(2)$-equivariant anti-self-dual connections on $\mathbb{R}^{4}$.

Let $\rho_{i}$ be the unique $i$-dimensional irreducible representation of SU(2). Consider $\mathrm{SU}(2)$-modules $V, W$ defined by

$$
V=\bigoplus V_{i} \otimes \rho_{i}, \quad W=\bigoplus W_{i} \otimes \rho_{i} .
$$

Then $M=M(\mathbf{v}, \mathbf{w})$ can be viewed as the $\mathrm{SU}(2)$-invariant part of $\operatorname{Hom}\left(V, \rho_{2} \otimes\right.$ $V) \oplus \operatorname{Hom}(V, W) \oplus \operatorname{Hom}(W, V)$. Here we use the Clebsch-Gordan rule: $\rho_{2} \otimes \rho_{i}=$ $\rho_{i-1} \oplus \rho_{i+1}$. Via the ADHM construction on $\mathbb{R}^{4}, \mathfrak{M}_{0}^{\text {reg }}$ can be regarded as a moduli space of SU(2)-equivariant instantons on a vector bundle $E$ over $\mathbb{R}^{4}$. The vectors $\mathbf{v}, \mathbf{w}$ determine the $\mathrm{SU}(2)$-module structures of the fibers $E_{0}, E_{\infty}$ over 0 , $\infty$. An SU(2)-equivariant anti-self-dual connection can be described as a solution of the ordinary differential equation, and thereby the main theorem of [ $\mathrm{Kr} 2]$ deduces our assertion.

Remarks 8.5. (1) $\mathfrak{M}_{0}^{\mathrm{reg}} \neq \varnothing$ if and only if $\mathcal{O}_{\lambda} \subset \overline{\mathcal{O}_{\mu}}$.

(2) Using 8.4 for each stratum, one can show that

$$
\mathfrak{M}_{0}=\overline{\mathcal{O}_{\mu}} \cap \mathscr{S}_{\lambda}
$$

(3) If $\mathbf{w}=(r, 0, \ldots, 0), \mathscr{S}_{\lambda}$ is all of $\operatorname{End}\left(\mathbb{C}^{r}\right)$. So in this case $\mathfrak{M}_{0}=\overline{\mathcal{O}_{\mu}}$. We thus recover the result of [KP].

(4) The reader may wonder why we treat only the type $A_{n}$. Kronheimer's result holds for any simple Lie algebra. In fact, we can describe $\mathcal{O}_{\mu} \cap \mathscr{S}_{\lambda}$ as a hyperKähler quotient of a finite-dimensional vector space, at least for classical Lie algebras using the same method. But it is different from our objects, quivers on graphs.

(5) If we use the holomorphic description $\mathfrak{M}_{0}=\mu_{\mathbb{C}}^{-1}(0) / / G_{\mathbf{v}}^{\mathbb{C}}$, both $\mathfrak{M}_{0}$ and $\overline{\mathcal{O}_{\mu}} \cap$ $\mathscr{S}_{\lambda}$ make sense over any field. Although our proof works only for $\mathbb{C}$, the result should hold in any algebraically closed field. 
In $\S 7$, we showed that $\mathfrak{M}_{\left(\zeta_{\mathbb{R}}, 0\right)}(\mathbf{v}, \mathbf{w})$ is isomorphic to $T^{*} \mathscr{F}=T^{*} \mathscr{F}\left(v_{1}, \ldots, v_{n} ; r\right)$ if $\mathbf{w}=(r, 0, \ldots, 0)$. Let us denote by $\Pi$ the projection to the second factor in the description (7.1). Its image is $\overline{\mathcal{O}}_{\mu}$, and $\Pi: T^{*} \mathscr{F} \rightarrow \overline{\mathcal{O}}_{\mu}$ is a resolution of singularities. We conjecture the following for general data $\mathbf{v}, \mathbf{w}$.

CONJECTURe 8.6. Suppose that $\left(\zeta_{\mathbb{R}}, 0\right)$ is generic and $\mathfrak{M}_{0}^{\mathrm{reg}}(\mathbf{v}, \mathbf{w})$ is nonempty. Define $\lambda, \mu$ by (8.2), (8.3). Then there is the following diagram:

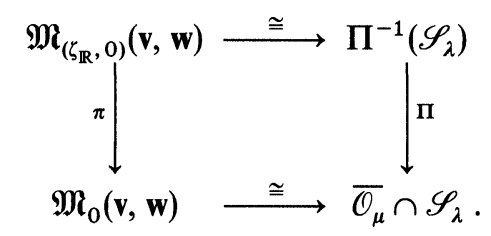

In $\S 5$ we observed that $\mathfrak{M}_{\left(\zeta_{\mathbb{R}}, 0\right)}$ contains a subvariety $\pi^{-1}(0)$ as a deformation retract. It should correspond to $\Pi^{-1}\left(N_{\lambda}\right)=\Pi^{-1}\left(\mathcal{O}_{\lambda} \cap \mathscr{S}_{\lambda}\right)$. This variety is called Spaltenstein's variety and was extensively studied by Borho-MacPherson [BM] $\left(\mathscr{P}_{x}^{0}\right.$ in their notation). They computed its Poincaré polynomial. Combining results in $\S 6$ and $\S 7$, we have the following.

Proposition 8.7. Under the same assumption as in (8.6), there exists an isomorphism

$$
H^{*}\left(\mathfrak{M}_{\left(\zeta_{\mathbb{R}}, 0\right)}(\mathbf{v}, \mathbf{w}) ; \mathbb{Z}\right) \cong H^{*}\left(\Pi^{-1}\left(N_{\lambda}\right) ; \mathbb{Z}\right)
$$

9. Monodromy representations of the Weyl groups. The purpose of this section is to define the monodromy representation of the Weyl group on the homology of quiver variety in the case when the graph is of Dynkin type. Here we shall give a geometric definition which relies on the result in [KN]. There is a purely algebraic definition using "the reflection functor", which can be applied to any graphs. The details will be given elsewhere. The results of this section will not be used in other sections.

Let $\left(\mathbb{R}^{3} \otimes Z\right)^{\circ}$ be the set of generic parameters $\zeta=\left(\zeta_{\mathbb{R}}, \zeta_{\mathbb{C}}\right)$. (We fix dimension vectors $\mathbf{v}, \mathbf{w}$.) Then $\left(\mathbb{R}^{3} \otimes Z\right)^{\circ}$ is simply connected, since the complement is the union of real codimension-3 subspaces. So the local system of the cohomology $H^{*}\left(\mathfrak{M}_{\zeta} ; \mathbb{R}\right)$ of the moduli spaces is trivialized over $\left(\mathbb{R}^{3} \otimes Z\right)^{\circ}$. It is also known that the cohomology of the ALE space $H^{2}\left(X_{\zeta} ; \mathbb{R}\right)$ is isomorphic to the parameter space $\mathbb{R}^{n}$ and is identified with the Cartan subalgebra $\mathfrak{h}$ of the simple Lie algebra corresponding to the given Dynkin graph. (See [Kr1, §4].)

Let $W$ be the Weyl group associated with the given Dynkin graph. It is a subgroup of $\mathrm{GL}(\mathfrak{h}) \cong \mathrm{GL}(n ; \mathbb{R})$ generated by reflections in hyperplanes defined by roots.

Proposition 9.1. Suppose that $\zeta$ is generic. If $\zeta^{\prime}=\sigma \zeta$ for some $\sigma \in W$, there exists a hyper-Kähler isometry $\Phi_{\sigma}: \mathfrak{M}_{\zeta}(\mathbf{v}, \mathbf{w}) \rightarrow \mathfrak{M}_{\zeta^{\prime}}\left(\mathbf{v}^{\prime}, \mathbf{w}\right)$ where $\mathbf{v}^{\prime}$ is given by the 
formula $\sigma(\mathbf{w}-\mathbf{C v})=\mathbf{w}-\mathbf{C v}^{\prime}$. Here $\mathbf{C}=\left(c_{k l}\right)$ is the positive definite Cartan matrix associated with the given Dynkin graph. Moreover it holds $\Phi_{\sigma} \circ \Phi_{\tau}=\Phi_{\sigma \tau}$.

Proof. The results are known for the ALE space: it is known that if $\zeta^{\prime}=\sigma \zeta$ for some $\sigma \in W$, there exists a hyper-Kähler isometry $f_{\sigma}: X_{\zeta} \rightarrow X_{\zeta^{\prime}}$. We also have $f_{\sigma} \circ f_{\tau}=f_{\sigma \tau}$. It is also known that $f_{\sigma}$ induces $\sigma^{-1}$ on $H^{2}\left(X_{\zeta} ; \mathbb{R}\right) \cong \mathfrak{h}$ via the above trivialization $[\mathrm{Kr} 1, \S 4]$. The map $f_{\sigma}$ induces a map $\Psi_{\sigma}: \mathfrak{M}_{\zeta^{\prime}}(E) \rightarrow \mathfrak{M}_{\zeta}\left(f_{\sigma}^{*}(E)\right)$ by pulling back connections. The framing at infinity (or the flat connection on the end) is unchanged, and hence the dimension vector $\mathbf{w}$ of $W$ is the same for $E$ and $f_{\sigma}^{*}(E)$.

Let $\mathscr{R}_{l}^{\zeta}$ denote the tautological vector bundle over $X_{\zeta}$ (see $\left.\S 2\right)$. Then it depends smoothly on $\zeta$, so $c_{1}\left(\mathscr{R}_{l}^{\zeta}\right)$ is independent of $\zeta$ if we identify $H^{2}\left(X_{\zeta} ; \mathbb{R}\right)$ with $\mathfrak{h}$ as above. Let $\mathbf{v}$ (resp. $\left.\mathbf{v}^{\prime}\right)$ be the dimension vector corresponding to $\mathfrak{M}_{\zeta}\left(f_{\sigma}^{*}(E)\right.$ ) (resp. $\mathfrak{M}_{\zeta^{\prime}}(E)$ ). The condition $V_{0}=0$ (see Remark 2.15) can be expressed in terms of the index theorem (see [KN, (A.1)]), and it can be checked that the condition is preserved. The vector $\mathbf{u}^{\prime}=\mathbf{w}-\mathbf{C v}^{\prime}=\left(u_{1}^{\prime}, u_{2}^{\prime}, \ldots, u_{n}^{\prime}\right)$ is given by [KN, 9.3]

$$
c_{1}(E)=\sum u_{l}^{\prime} c_{1}\left(\mathscr{R}_{l}^{\zeta^{\prime}}\right)
$$

Since $f_{\sigma}^{*}$ acts on $H^{2}\left(X_{\zeta} ; \mathbb{R}\right) \cong \mathfrak{h}$ by $\sigma^{-1}$, we have

$$
c_{1}\left(f^{*}(E)\right)=\sum u_{l} c_{1}\left(\mathscr{R}_{l}^{\zeta}\right),
$$

where

$$
\left(u_{1}, \ldots, u_{n}\right)^{t}=\mathbf{u}=\sigma^{-1} \mathbf{u}^{\prime}=\sigma^{-1}\left(u_{1}^{\prime}, \ldots, u_{n}^{\prime}\right)^{t}
$$

Now $\Phi_{\sigma} \stackrel{\text { def. }}{=} \Psi_{\sigma}^{-1}$ satisfies the desired properties.

Remark 9.2. By Proposition 9.1, we may assume that the vector $\mathbf{u}$ is in a chosen Weyl chamber, for example $u_{k} \geqslant 0(1 \leqslant k \leqslant n)$. When $\mathbf{w}-\mathbf{C v}=0$ (i.e., $\left.c_{1}(E)=0\right)$, the above gives a map $\mathfrak{M}_{\zeta}(\mathbf{v}, \mathbf{w}) \rightarrow \mathfrak{M}_{\sigma \zeta}(\mathbf{v}, \mathbf{w})$. Since the local system of the cohomology $H^{*}\left(\mathfrak{M}_{\zeta}(\mathbf{v}, \mathbf{w}) ; \mathbb{R}\right)$ is trivialized over $\left(\mathbb{R}^{3} \otimes Z\right)^{\circ}$, we have the following.

COROllaRY 9.3. There exists a Weyl group representation on $H^{*}\left(\mathfrak{M}_{\zeta}(\mathbf{v}, \mathbf{w}) ; \mathbb{Z}\right)$ when $\mathbf{w}-\mathbf{C v}=0$.

This is the analogue of the Weyl group representation defined by Slodowy [S1].

10. Representations of Kac-Moody algebras. Throughout this section, we assume that $\zeta=\left(\zeta_{\mathbb{R}}, 0\right)$ and $\zeta_{\mathbb{R}}$ satisfies the condition (3.4). We first give a construction of representation of enveloping algebras in terms of $\mathfrak{M}_{\zeta}$. Our idea is motivated by Lusztig's construction [L3, 12.10].

Let $u$ be the universal enveloping algebra of the Kac-Moody algebra attached 
to the (symmetric) generalized Cartan matrix $\mathbf{C}=\left(c_{k l}\right)_{1 \leqslant k, l \leqslant n}$. It is the algebra over $\mathbb{Q}$ with 1 generated by $E_{k}, F_{k}, H_{k}(1 \leqslant k \leqslant n)$ satisfying the following relations:

$$
\begin{gathered}
H_{k} H_{l}=H_{l} H_{k}, \\
H_{k} E_{l}-E_{l} H_{k}=c_{k l} E_{l}, \quad H_{k} F_{l}-F_{l} H_{k}=-c_{k l} F_{l}, \\
E_{k} F_{l}-F_{l} E_{k}=\delta_{k l} H_{k}, \\
\sum_{p=0}^{1-c_{k l}}(-1)^{p}\left(\begin{array}{c}
1-c_{k l} \\
p
\end{array}\right) E_{k}^{p} E_{l} E_{k}^{1-c_{k l}-p}=0 \quad(k \neq l), \\
\sum_{p=0}^{1-c_{k l}}(-1)^{p}\left(\begin{array}{c}
1-c_{k l} \\
p
\end{array}\right) F_{k}^{p} F_{l} F_{k}^{1-c_{k l}-p}=0 \quad(k \neq l) .
\end{gathered}
$$

(Our definition is different from that in $[\mathrm{Kac}]$ when $\operatorname{det} \mathbf{C}=0$. Our algebra is denoted by $U\left(\mathfrak{g}^{\prime}\right)$ in $[\mathrm{Kac}]$.)

Let $u^{-}$be the $\mathbb{Q}$-subalgebra of $u$ generated by the elements $F_{k}(1 \leqslant k \leqslant n)$. Let $L(\mathbf{w})$ be the irreducible highest-weight integrable representation of $u$ with highest weight $\mathbf{w}$. It has a nonzero vector $x$ such that

(1) $E_{k} x=0, H_{k} x=w_{k} x$ for all $k$,

(2) the map $\pi: u^{-1} \rightarrow L(\mathbf{w}) ; \alpha \mapsto \alpha x$ is surjective and its kernel is the left ideal in $u^{-}$generated by $F_{k}^{w_{k}+1}(1 \leqslant k \leqslant n)$.

For a complex variety $X$ let $M(X)$ denote the $\mathbb{Q}$-vector space consisting of all constructible functions $f: X \rightarrow \mathbb{Q}$. When $X=\varnothing$, we set $M(X)=0$. For any holomorphic $p: X \rightarrow X^{\prime}$ we define linear maps $p^{*}: M\left(X^{\prime}\right) \rightarrow M(X)$ and $p_{!}: M(X) \rightarrow$ $M\left(X^{\prime}\right)$ by $\left(p^{*} f^{\prime}\right)(x) \stackrel{\text { def. }}{=} f^{\prime}(p(x)),\left(p_{1} f\right)(x) \stackrel{\text { def. }}{=} \sum_{a \in \mathbb{Q}} a \chi\left(p^{-1}(x) \cap f^{-1}(a)\right)$, where $\chi$ denotes Euler characteristic of cohomology with compact support. (See [Mc].)

Let $\Lambda(\mathbf{v}, \mathbf{w}) \stackrel{\text { def. }}{=}\left\{(B, 0, j) \in \mu_{\mathbb{C}}^{-1}(0) \mid 0 \in \overline{G_{\mathbf{v}}^{\mathbb{C}} B}\right\}$ as in Remark 5.11. $(\Lambda(\mathbf{v}, 0)$ is denoted by $\Lambda_{v}$ in $[\mathrm{L} 3,12.1]$.)

Let $C=\left(C_{k}\right)_{1 \leqslant k \leqslant n}$ be a collection of subspaces $C_{k}$ of $V_{k}$, and let $(B, 0, j) \in$ $\Lambda(\mathbf{v}, \mathbf{w})$. We say $C$ is $B$-stable if $B_{h}\left(C_{\text {out }(h)}\right) \subset C_{\text {in }(h)}$ for any $h \in H$.

Let $\mathbf{w}, \mathbf{v}, \mathbf{v}^{\prime}, \mathbf{v}^{\prime \prime} \in \mathbb{Z}_{\geqslant 0}^{n}$ be such that $\mathbf{v}=\mathbf{v}^{\prime}+\mathbf{v}^{\prime \prime}$. We consider the diagram

$$
\Lambda\left(\mathbf{v}^{\prime \prime}, 0\right) \times \Lambda\left(\mathbf{v}^{\prime}, \mathbf{w}\right) \stackrel{p_{1}}{\leftarrow} \tilde{\mathbf{F}}\left(\mathbf{v}, \mathbf{w} ; \mathbf{v}^{\prime \prime}\right) \stackrel{p_{2}}{\rightarrow} \mathbf{F}\left(\mathbf{v}, \mathbf{w} ; \mathbf{v}^{\prime \prime}\right) \stackrel{p_{3}}{\rightarrow} \Lambda(\mathbf{v}, \mathbf{w}),
$$

where the notations are as follows. A point of $\mathbf{F}\left(\mathbf{v}, \mathbf{w} ; \mathbf{v}^{\prime \prime}\right)$ is a point $(B, 0, j) \epsilon$ $\Lambda(\mathbf{v}, \mathbf{w})$ together with a collection of subspaces $C_{k}$ of $V_{k}$ for each vertex $k$ such that $C=\left(C_{k}\right)_{k}$ is $B$-stable and has the dimension $\mathbf{v}^{\prime}=\mathbf{v}-\mathbf{v}^{\prime \prime}$. A point of $\tilde{\mathbf{F}}\left(\mathbf{v}, \mathbf{w} ; \mathbf{v}^{\prime \prime}\right)$ is a point $(B, 0, j, C)$ of $\mathbf{F}\left(\mathbf{v}, \mathbf{w} ; \mathbf{v}^{\prime \prime}\right)$ together with a collection of isomorphisms $R_{k}^{\prime}: V_{k}^{\prime} \cong$ $C_{k}, R_{k}^{\prime \prime}: V_{k}^{\prime \prime} \cong V_{k} / C_{k}$ for each vertex $k$. Let us define $p_{2}\left(B, 0, j, C, R^{\prime}, R^{\prime \prime}\right)=$ $(B, 0, j, C), p_{3}(B, 0, j, C)=(B, 0, j)$. Define the map $p_{1}$ by $p_{1}\left(B, 0, j, C, R^{\prime}, R^{\prime \prime}\right)=$ 
$\left(B^{\prime \prime}, B^{\prime}, 0, j^{\prime}\right)$ where $B^{\prime \prime}, B^{\prime}, j^{\prime}$ are detetmined by

$$
\begin{aligned}
R_{\mathrm{in}(h)}^{\prime} B_{h}^{\prime} & =B_{h} R_{\mathrm{out}(h)}^{\prime}: V_{\mathrm{out}(h)}^{\prime} \rightarrow C_{\mathrm{in}(h)}, \\
j_{k}^{\prime} & =j_{k} R_{k}^{\prime}: V_{k}^{\prime} \rightarrow W_{k}, \\
R_{\mathrm{in}(h)}^{\prime \prime} B_{h}^{\prime \prime} & =B_{h} R_{\mathrm{out}(h)}^{\prime \prime}: V_{\mathrm{out}(h)}^{\prime \prime} \rightarrow V_{\mathrm{in}(h)} / C_{\mathrm{in}(h)} .
\end{aligned}
$$

Nilpotency of $B^{\prime}, B^{\prime \prime}$ can be checked as follows. As in the proof of Lemma 5.9, there exists a filtration $0=V_{k}^{(0)} \subset V_{k}^{(1)} \subset \cdots \subset V_{k}^{(N)}=V_{k}$ such that $B_{h}\left(V_{\text {out }(h)}^{(m)}\right) \subset$ $V_{\text {in }(h)}^{(m-1)}$. Then

$$
\begin{gathered}
R_{\mathrm{in}(h)}^{\prime} B_{h}^{\prime} R_{\mathrm{out}(h)}^{\prime-1}\left(V_{\mathrm{out}(h)}^{(m)} \cap C_{\mathrm{out}(h)}\right) \subset V_{\mathrm{in}(h)}^{(m-1)} \cap C_{\mathrm{in}(h)}, \\
R_{\mathrm{in}(h)}^{\prime \prime} B_{h}^{\prime \prime} R_{\mathrm{out}(h)}^{\prime \prime-1}\left(V_{\mathrm{out}(h)}^{(m)} / V_{\mathrm{out}(h)}^{(m)} \cap C_{\mathrm{out}(h)}\right) \subset V_{\mathrm{in}(h)}^{(m-1)} / V_{\mathrm{in}(h)}^{(m-1)} \cap C_{\mathrm{in}(h)} .
\end{gathered}
$$

This implies the nilpotency.

Recall that we have

$$
\begin{aligned}
H_{\zeta}^{\mathrm{s}} & \cap \Lambda(\mathbf{v}, \mathbf{w}) \\
& =\{(B, 0, j) \in \Lambda(\mathbf{v}, \mathbf{w}) \mid(B, 0, j) \text { satisfies the condition (1) in Proposition 3.5 }\}
\end{aligned}
$$

Then the following is obvious.

LEMMA 10.3. In the diagram (10.2), we have

$$
\left(p_{3} \circ p_{2}\right)^{-1}\left(\Lambda(\mathbf{v}, \mathbf{w}) \cap H_{\zeta}^{\mathrm{s}}\right) \subset p_{1}^{-1}\left(\Lambda\left(\mathbf{v}^{\prime \prime}, 0\right) \times\left(\Lambda\left(\mathbf{v}^{\prime}, \mathbf{w}\right) \cap H_{\zeta}^{\mathrm{s}}\right)\right) .
$$

By this lemma, we can restrict the diagram (10.2) to $H_{\zeta}^{\text {s }}$, forget the $\Lambda(\mathbf{v}, \mathbf{w})$ factor, and consider its quotient by $G_{\mathbf{v}}^{\mathbb{C}}, G_{\mathbf{v}^{\prime}}^{\mathbb{C}}$. We get the diagram

$$
\mathfrak{L}\left(\mathbf{v}^{\prime}, \mathbf{w}\right) \stackrel{\pi_{1}}{\leftarrow} \mathfrak{F}\left(\mathbf{v}, \mathbf{w} ; \mathbf{v}-\mathbf{v}^{\prime}\right) \stackrel{\pi_{2}}{\rightarrow} \mathfrak{L}(\mathbf{v}, \mathbf{w}),
$$

where

$$
\mathfrak{F}\left(\mathbf{v}, \mathbf{w} ; \mathbf{v}-\mathbf{v}^{\prime}\right) \stackrel{\text { def. }}{=}\left\{(B, 0, j, C) \in \mathbf{F}\left(\mathbf{v}, \mathbf{w} ; \mathbf{v}-\mathbf{v}^{\prime}\right) \mid(B, 0, j) \in H_{\zeta}^{\mathrm{s}}\right\} / G_{\mathbf{v}}^{\mathbb{C}}
$$

If we invoke the proof of the ADHM description [KN], we can see the gauge theoretic meaning of $\mathfrak{F}\left(\mathbf{v}, \mathbf{w} ; \mathbf{v}-\mathbf{v}^{\prime}\right)$ : Consider the ALE space $X_{\zeta}$ as the minimal resolution of the simple singularity as in $\S 2$. Then $\mathfrak{F}\left(\mathbf{v}, \mathbf{w} ; \mathbf{v}-\mathbf{v}^{\prime}\right)$ is the moduli space of pairs of holomorphic vector bundles and subsheaves of their restriction to the exceptional set. The dimension data $\mathbf{v}, \mathbf{w}$ describes the Chern class and the rank of the vector bundle, while $\mathbf{v}^{\prime}$ gives the ranks of subsheaves on 
irreducible components of the exceptional set. Hence $\mathfrak{F}\left(\mathbf{v}, \mathbf{w} ; \mathbf{v}-\mathbf{v}^{\prime}\right)$ is an analogue of the Hecke correspondence (see $\$ 12$ and [La]). It plays a crucial role in our construction.

For a later purpose we introduce a generalization of the variety $\mathfrak{F}\left(\mathbf{v}, \mathbf{w} ; \mathbf{v}^{\prime}\right)$. Let $(B, 0, j) \in \Lambda(\mathbf{v}, \mathbf{w})$. Let $\left\{C_{k}^{1} \supset C_{k}^{2} \supset \cdots \supset C_{k}^{r}\right\}_{k}$ be a collection of flags in $V_{k}$ for all vertices $k$. It is said to be $B$-stable, if $\left(C_{k}^{a}\right)_{k}$ is $B$-stable for all $a=1, \ldots, r$. Let $\mathbf{F}\left(\mathbf{v}, \mathbf{w} ; \mathbf{v}^{1}, \mathbf{v}^{2}, \ldots, \mathbf{v}^{r}\right)$ be the variety of all pairs of $(B, 0, j) \in \Lambda(\mathbf{v}, \mathbf{w})$ and $B$-stable flags $\left\{C_{k}^{1} \supset C_{k}^{2} \supset \cdots \supset C_{k}^{r}\right\}_{k}$ such that the dimension of $C^{a}$ is $\mathbf{v}-\sum_{i \leqslant a} \mathbf{v}^{i}$. Let us define

$$
\begin{aligned}
& \mathfrak{F}\left(\mathbf{v}, \mathbf{w} ; \mathbf{v}^{1}, \mathbf{v}^{2}, \ldots, \mathbf{v}^{r}\right) \\
& \quad \stackrel{\text { def. }}{=}\left\{\left(B, 0, j ;\left\{C_{k}^{1} \supset \cdots \supset C_{k}^{r}\right\}_{k}\right) \in \mathbf{F}\left(\mathbf{v}, \mathbf{w} ; \mathbf{v}^{1}, \mathbf{v}^{2}, \ldots, \mathbf{v}^{r}\right) \mid(B, 0, j) \in H_{\zeta}^{\mathbf{s}}\right\} / G_{\mathbf{v}}^{\mathbb{C}}
\end{aligned}
$$

There is a diagram

$$
\mathfrak{L}\left(\mathbf{v}-\sum_{a=1}^{r} \mathbf{v}^{a}, \mathbf{w}\right) \stackrel{\pi_{1}}{\leftarrow} \mathfrak{F}\left(\mathbf{v}, \mathbf{w} ; \mathbf{v}^{1}, \mathbf{v}^{2}, \ldots, \mathbf{v}^{r}\right) \stackrel{\pi_{2}}{\rightarrow} \mathfrak{L}(\mathbf{v}, \mathbf{w})
$$

as in (10.4). The map $\pi_{1}$ is given by the restriction of $(B, 0, j)$ to $C^{r}$, and $\pi_{2}$ is to take the first component $(B, 0, j)$.

For each vertex $k$, let $\mathbf{e}^{k}$ be the vector given by

$$
\mathbf{e}^{k}={ }^{t}\left(e_{1}^{k}, \ldots, e_{n}^{k}\right) ; \quad e_{l}^{k}=\delta_{k l} .
$$

We define the following operators on the vector space $M(\mathfrak{L}(\mathbf{v}, \mathbf{w}))$ of all constructible functions on $\mathfrak{Q}(\mathbf{v}, \mathbf{w})$ :

$$
\begin{array}{ll}
H_{k}: M(\mathfrak{L}(\mathbf{v}, \mathbf{w})) \rightarrow M(\mathfrak{L}(\mathbf{v}, \mathbf{w})) ; & H_{k} f=u_{k} f \\
E_{k}: M(\mathfrak{L}(\mathbf{v}, \mathbf{w})) \rightarrow M\left(\mathfrak{I}\left(\mathbf{v}-\mathbf{e}^{k}, \mathbf{w}\right)\right) ; & E_{k} f=\left(\pi_{1}\right)_{!}\left(\pi_{2}^{*} f\right) \\
F_{k}: M\left(\mathfrak{L}\left(\mathbf{v}-\mathbf{e}^{k}, \mathbf{w}\right)\right) \rightarrow M(\mathfrak{L}(\mathbf{v}, \mathbf{w})) ; & F_{k} g=\left(\pi_{2}\right)_{!}\left(\pi_{1}^{*} g\right),
\end{array}
$$

where we set $\mathbf{u}={ }^{t}\left(u_{1}, u_{2}, \ldots, u_{n}\right)=\mathbf{w}-\mathbf{C v}$ and consider the diagram (10.4) with $\mathbf{v}^{\prime}=\mathbf{v}-\mathbf{e}^{k}$ in the definition of $E_{k}, F_{k}$. These are "geometric Hecke operators".

Then the following is easy to check.

LEMMA 10.6. Let $\left(i_{1}, i_{2}, \ldots, i_{r}\right)$ be a sequence of vertices, and take $\mathbf{v} \in \mathbb{Z}_{\geqslant 0}^{n}$. Then we have

$$
E_{i_{r}} E_{i_{r-1}} \cdots E_{i_{1}} f=\left(\pi_{1}\right)_{!}\left(\pi_{2}^{*} f\right), \quad F_{i_{1}} F_{i_{2}} \cdots F_{i_{r}} g=\left(\pi_{2}\right)_{!}\left(\pi_{1}^{*} g\right),
$$

for $f \in M(\mathfrak{L}(\mathbf{v}, \mathbf{w})), g \in M\left(\mathfrak{I}\left(\mathbf{v}-\sum_{a=1}^{r} \mathbf{v}^{a}, \mathbf{w}\right)\right)$. Here we have used the diagram (10.5) with $\mathbf{v}^{a}=\mathbf{e}^{i_{a}}$. 
LEMMA 10.7. The operators $E_{k}, F_{k}$ on $\bigoplus_{\mathbf{v}} M(\mathfrak{L}(\mathbf{v}, \mathbf{w}))$ satisfy the relations (10.1.d), (10.1.e).

Proof. The relation (10.1.e) was proved by Lusztig [L3, 12.13]. We reproduce the proof in our notation. It also implies (10.1.d) at the same time.

Fix vertices $k, l$ with $k \neq l$, and set $N \stackrel{\text { def. }}{=}-c_{k l}$. For $p=0,1, \ldots, N+1$, consider four vectors $\mathbf{v}, \mathbf{v}^{1}=p \mathbf{e}^{l}, \mathbf{v}^{2}=\mathbf{e}^{k}, \mathbf{v}^{3}=(N+1-p) \mathbf{e}^{l}$ and the corresponding diagram (10.5). Then, using (10.6) and the fact that the Euler characteristic of the variety consisting of the complete flags $0=C^{0} \varsubsetneqq C^{1} \cdots \varsubsetneqq C^{r-1} \varsubsetneqq C^{r}=\mathbb{C}^{r}$ in $\mathbb{C}^{r}$ is equal to $r$ !, we get

$$
\frac{E_{l}^{N+1-p}}{(N+1-p) !} E_{k} \frac{E_{l}^{p}}{p !} f=\left(\pi_{1}\right)_{!}\left(\pi_{2}^{*} f\right), \quad \frac{F_{l}^{p}}{p !} F_{k} \frac{F_{l}^{N+1-p}}{(N+1-p) !} g=\left(\pi_{2}\right)_{!}\left(\pi_{1}^{*} f\right) .
$$

Let $\mathbf{v}^{\prime}=\mathbf{v}^{1}+\mathbf{v}^{2}+\mathbf{v}^{3}=\mathbf{e}^{k}+(N+1) \mathbf{e}^{l}$. The maps $\pi_{1}, \pi_{2}$ factor through $\mathfrak{F}\left(\mathbf{v}, \mathbf{w} ; \mathbf{v}^{\prime}\right)$ :

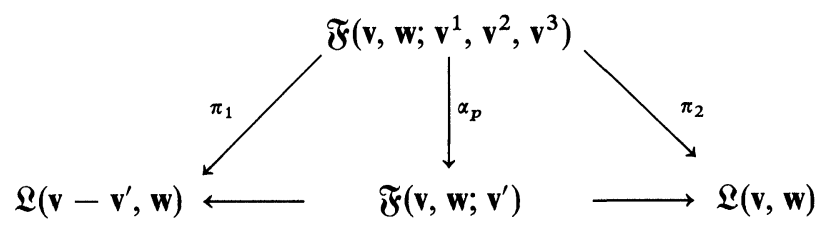

where $\alpha_{p}$ is defined by forgetting $C^{1}, C^{2}$. Note that $\mathfrak{F}\left(\mathbf{v}, \mathbf{w} ; \mathbf{v}^{\prime}\right)$ is independent of $p$.

To show (10.1.d) it is enough to check

$$
\sum_{p=0}^{N+1}(-1)^{p} \chi\left(\alpha_{p}^{-1}(x)\right)=0
$$

for any $x \in \mathfrak{F}\left(\mathbf{v}, \mathbf{w} ; \mathbf{v}^{\prime}\right)$.

Take a representative $\left(B, 0, j, C^{3}\right)$ of $x$. Then the fiber of $\alpha_{p}^{-1}(x)$ is isomorphic to the variety of all codimension- $p$ subspaces $C_{l}^{1}$ such that

$$
C_{l}^{3}+\sum_{h: \operatorname{in}(h)=l} \operatorname{im} B_{h} \subset C_{l}^{1} \subset \sum_{h: \operatorname{in}(h)=k, \text { out }(h)=l} B_{h}^{-1}\left(C_{k}^{3}\right) .
$$

In fact, if the above $C_{l}^{1}$ is given, $C^{1}, C^{2}$ are given by

$$
C_{m}^{1}=V_{m} \quad \text { for } m \neq l, \quad C_{m}^{2}= \begin{cases}C_{k}^{3} & \text { if } m=k \\ C_{m}^{1} & \text { otherwise } .\end{cases}
$$

The condition (10.9) is equivalent to saying $C^{1}, C^{2}$ are $B$-stable.

Let $S_{1}$ (resp. $S_{2}$ ) be the left-hand (resp. right-hand) side of (10.9). We first show that $S_{1} \subset S_{2}$. Since $C^{3}$ is $B$-stable, we have $B_{h}\left(C_{l}^{3}\right) \subset C_{k}^{3}$ for all $h \in H$ such that $\operatorname{out}(h)=l, \operatorname{in}(h)=k$. So it is enough to show that $B_{h} B_{h^{\prime}}\left(V_{\text {out }\left(h^{\prime}\right)}\right) \subset C_{k}^{3}$ for all $h$, 
$h^{\prime} \in H$ such that $\operatorname{in}(h)=k$, out $(h)=\operatorname{in}\left(h^{\prime}\right)=l$. When out $\left(h^{\prime}\right) \neq k$, we have

$$
B_{h} B_{h^{\prime}}\left(V_{\text {out }\left(h^{\prime}\right)}\right)=B_{h} B_{h^{\prime}}\left(C_{\text {out }\left(h^{\prime}\right)}^{3}\right) \subset B_{h}\left(C_{\operatorname{in}\left(h^{\prime}\right)}^{3}\right) \subset C_{k}^{3} .
$$

Thus we may assume out $\left(h^{\prime}\right)=k$.

As in the proof of (5.9), we can take a flag $0=V_{m}^{(0)} \subset V_{m}^{(1)} \subset V_{m}^{(2)} \subset \cdots \subset$ $V_{m}^{(N)}=V_{m}$ such that $B_{h}\left(V_{\text {out }(h)}^{(m)}\right) \subset V_{\operatorname{in}(h)}^{(m-1)}$. Since $C_{k}^{3}$ is codimension-1 in $V_{k}$, there exists an $m_{0}$ such that

$$
V_{k}^{\left(m_{0}-1\right)} \subset C_{k}^{3}, \quad V_{k}^{\left(m_{0}\right)} / V_{k}^{\left(m_{0}\right)} \cap C_{k}^{3}=V_{k} / C_{k}^{3} .
$$

Since we have $B_{h} B_{h^{\prime}}\left(V_{k}^{\left(m_{0}\right)}\right) \subset V_{k}^{\left(m_{0}-2\right)} \subset C_{k}^{3}$, the second equality in the above and the $B$-stability of $C^{3}$ implies $B_{h} B_{h^{\prime}}\left(V_{k}\right) \subset C_{k}^{3}$. Hence $S_{1} \subset S_{2}$.

Next we observe that $S_{1} \neq S_{2}$. Let us define a subset $H^{\prime} \subset H$ by $h \in H^{\prime}$ if and only if out $(h)=k, \operatorname{in}(h)=l$. We have the diagram

$$
0 \rightarrow V_{l} / S_{2} \rightarrow V_{k} / C_{k}^{3} \oplus \cdots \oplus V_{k} / C_{k}^{3} \rightarrow S_{1} / C_{l}^{3} \rightarrow 0,
$$

where the middle term is a direct sum of copies of $V_{k} / C_{k}^{3}$, one for each $h \in H^{\prime}$; the second arrow is defined by $v \mapsto\left(B_{\bar{h}}(v)\right)_{h \in H^{\prime}}$; the third arrow is $\left(v_{h}\right)_{h \in H^{\prime}} \mapsto$ $\sum_{h \in H^{\prime}} \varepsilon(h) B_{h}\left(v_{h}\right)$. The complex ADHM equation implies that the diagram is complex. It is clear that the diagram is acyclic except possibly at the middle. Hence we have $\operatorname{dim} V_{l} / S_{2} \leqslant N-\operatorname{dim} S_{1} / C_{l}^{3}$. Thus we get $\operatorname{dim} S_{2}-\operatorname{dim} S_{1} \geqslant \operatorname{dim} V_{l}-$ $\operatorname{dim} C_{l}^{3}-N=1$; in particular, $S_{1} \neq S_{2}$.

Let $d_{1}, d_{2}$ be the codimension of $S_{1}, S_{2}$ in $V_{l}$ respectively. We have $d_{2}<d_{1}$ by the above discussion. Then $\alpha_{p}(x)$ is empty unless $d_{2} \leqslant p \leqslant d_{1}$, in which case the fiber is a Grassmannian manifold of $\left(d_{1}-p\right)$-dimensional subspaces in a $\left(d_{1}-d_{2}\right)$-dimensional space. Hence (10.8) follows as

$$
\sum_{p=0}^{N+1}(-1)^{p} \chi\left(\alpha_{p}^{-1}(x)\right)=\sum_{p=d_{2}}^{d_{1}}(-1)^{p}\left(\begin{array}{c}
d_{1}-d_{2} \\
d_{1}-p
\end{array}\right)=0 .
$$

LEMMA 10.10. (1) Let $x \in \mathfrak{I}(\mathbf{v}, \mathbf{w})$, and let $(B, 0, j)$ be its representative. In the diagram (10.4) with $\mathbf{v}^{\prime}=\mathbf{v}-\mathbf{e}^{k}$, we have

$$
\pi_{2}^{-1}(x) \cong \mathbb{C P}^{d-1}, \quad d=\operatorname{dim} V_{k}-\operatorname{dim} \sum_{h \in H: \operatorname{in}(h)=k} \operatorname{im} B_{h}
$$

(2) Let $x \in \mathfrak{Q}(\mathbf{v}, \mathbf{w})$ and $\left(B^{\prime}, 0, j^{\prime}\right)$ be its representative. In the diagram (10.4) with $\mathbf{v}=\mathbf{v}^{\prime}-\mathbf{e}^{k}$, we have

$$
\begin{gathered}
\pi_{1}^{-1}(x) \cong \mathbb{C P}^{d^{\prime}-1}, \\
d^{\prime}=\operatorname{dim} W_{k}+\sum_{l} a_{k l} \operatorname{dim} V_{l}^{\prime}-\operatorname{dim} V_{k}^{\prime}-\operatorname{dim} \sum_{h \in H: \operatorname{in}(h)=k} \operatorname{im} B_{h}^{\prime} .
\end{gathered}
$$


Proof. (1) The fiber $\pi_{2}^{-1}(x)$ is isomorphic to the variety of all codimension-1 subspaces $C_{k}$ of $V_{k}$ such that im $B_{h} \subset C_{k}$ for all $h \in H$ with in $(h)=k$. Hence we have the assertion.

(2) First take collections $V, V^{\prime}$ of vector spaces with dimensions $\mathbf{v}, \mathbf{v}^{\prime}$ respectively. Fix an isomorphism $V_{k} \cong V_{k}^{\prime} \oplus \mathbb{C}$ and regard $V^{\prime}$ as a subspace of $V$.

Consider the space of all $(B, 0, j) \in \Lambda(\mathbf{v}, \mathbf{w})$ such that its restriction to $V^{\prime}$ is $\left(B^{\prime}, 0, j^{\prime}\right)$. It is isomorphic to the space $\mathbf{K}$ of all vectors

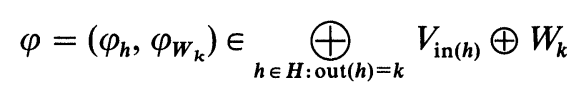

such that

$$
\sum_{h \in H: \text { out }(h)=k} \varepsilon(\bar{h}) B_{\bar{h}}^{\prime} \varphi_{h}=0 .
$$

Let $I$ be the image of

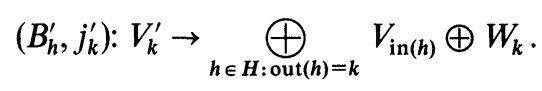

The complex ADHM equation implies $\mathbf{I} \subset \mathbf{K}$. We show that $(B, 0, j)$ satisfies the condition (3.5) (1) if and only if $\varphi \notin \mathbf{I}$. If $\varphi \in \mathbf{I}$, there exists a vector $v \in V_{k}^{\prime}$ such that $\left(B_{h}^{\prime}, j_{k}^{\prime}\right)(v)+\varphi=0$. Then $\mathbb{C}(v \oplus 1) \subset V_{k}^{\prime} \oplus \mathbb{C}=V_{k}$ violates the condition (3.5) (1). Suppose $\varphi \notin \mathbf{I}$ and there exists a $B$-stable collection $S=\left(S_{l}\right)_{l}$ of subspaces of $V_{l}$ with $j(S)=0$. Since $\left(B^{\prime}, 0, j^{\prime}\right)$ satisfies the condition (3.5) (1), we have $S \cap V^{\prime}=0$. In particular, $S$ has a nontrivial component only on the vertex $k$. Take $s \in S_{k}$ and write $s=\left(s^{\prime}, s^{\prime \prime}\right)$ according to the decomposition $V_{k}=V_{k}^{\prime} \oplus \mathbb{C}$. Then we have $\left(B_{h}^{\prime}, j_{k}^{\prime}\right)\left(s^{\prime}\right)+\varphi s^{\prime \prime}=0$. Since $\varphi \notin \mathbf{I}$ by the assumption, we must have $s^{\prime \prime}=0$. This means $S_{k} \subset V_{k}^{\prime}$; hence the condition (3.5) (1) for $\left(B^{\prime}, 0, j^{\prime}\right)$ implies $S_{k}=0$.

Taking $G_{\mathbf{v}}^{\mathbb{C}}$-action into consideration, we have that the fiber $\pi_{1}^{-1}(x)$ is isomorphic to the projective space $\mathbf{P}(\mathbf{K} / \mathbf{I})$.

The condition $(3.5)(1)$ for $\left(B^{\prime}, 0, j^{\prime}\right)$ implies $\left(B_{h}^{\prime}, j_{k}^{\prime}\right)$ is injective; hence $\operatorname{dim} \mathbf{I}=$ $\operatorname{dim} V_{k}^{\prime}$. On the other hand, we have

$$
\operatorname{dim} \mathbf{K}=\operatorname{dim} \operatorname{ker} \sum_{h \in H: \operatorname{out}(h)=k} \varepsilon(\bar{h}) B_{\bar{h}}^{\prime}+\operatorname{dim} W_{k} .
$$

Combining these, we have the assertion.

LEMMA 10.11. The linear operators $E_{k}, F_{k}, H_{k}$ on $\bigoplus_{\mathbf{v}} M(\mathcal{L}(\mathbf{v}, \mathbf{w}))$ satisfy the relations (10.1.c).

Proof. Fix vertices $k, l$, and take a vector $\mathbf{v} \in \mathbb{Z}_{\geqslant 0}^{n}$. Consider the space of all triples $\left((B, 0, j), C^{1}, C^{2}\right)$ where $(B, 0, j) \in \Lambda(\mathbf{v}, \mathbf{w}) \cap H_{\zeta}^{s}$ and $C^{1}, C^{2}$ are $B$-stable collections of subspaces of $V_{m}$ 's with the dimensions $\mathbf{v}-\mathbf{e}^{l}, \mathbf{v}-\mathbf{e}^{k}$ respectively. Let us denote by $\mathfrak{P}$ its quotient by $G_{\mathbf{v}}^{\mathbb{C}}$. It is isomorphic to the fiber product of $\mathfrak{F}\left(\mathbf{v}, \mathbf{w} ; \mathbf{e}^{k}\right)$ 
and $\mathfrak{F}\left(\mathbf{v}, \mathbf{w} ; \mathbf{e}^{l}\right)$ over $\mathfrak{L}(\mathbf{v}, \mathbf{w})$. There exists a diagram

$$
\mathfrak{L}\left(\mathbf{v}-\mathbf{e}^{l}, \mathbf{w}\right) \stackrel{\Pi_{1}}{\leftarrow} \mathfrak{P} \stackrel{\Pi_{2}}{\rightarrow} \mathfrak{L}\left(\mathbf{v}-\mathbf{e}^{k}, \mathbf{w}\right),
$$

and we have $E_{k} F_{l} f=\left(\Pi_{2}\right)_{!}\left(\Pi_{1}^{*} f\right)$.

Let us consider the fiber product of $\mathfrak{F}\left(\mathbf{v}-\mathbf{e}^{l}, \mathbf{w} ; \mathbf{e}^{k}\right)$ and $\mathfrak{F}\left(\mathbf{v}-\mathbf{e}^{k}, \mathbf{w} ; \mathbf{e}^{l}\right)$ over $\mathfrak{L}\left(\mathbf{v}-\mathbf{e}^{k}-\mathbf{e}^{l}, \mathbf{w}\right)$, namely

$$
\mathfrak{P}^{\prime}=\left\{\left(x^{1}, x^{2}\right) \in \mathfrak{F}\left(\mathbf{v}-\mathbf{e}^{l}, \mathbf{w} ; \mathbf{e}^{k}\right) \times \mathfrak{F}\left(\mathbf{v}-\mathbf{e}^{k}, \mathbf{w} ; \mathbf{e}^{l}\right) \mid \pi_{1}^{1}\left(x^{1}\right)=\pi_{1}^{2}\left(x^{2}\right)\right\},
$$

where we add the superscript 1,2 to $\pi_{1}$ in the diagram (10.4) in order to distinguish the maps for $\mathfrak{F}\left(\mathbf{v}-\mathbf{e}^{l}, \mathbf{w} ; \mathbf{e}^{k}\right)$ and $\mathfrak{F}\left(\mathbf{v}-\mathbf{e}^{k}, \mathbf{w} ; \mathbf{e}^{l}\right)$. There is a diagram

$$
\mathfrak{L}\left(\mathbf{v}-\mathbf{e}^{l}, \mathbf{w}\right) \stackrel{\Pi_{1}^{\prime}}{\leftarrow} \mathfrak{P}^{\prime} \stackrel{\Pi_{2}^{\prime}}{\rightarrow} \mathfrak{L}\left(\mathbf{v}-\mathbf{e}^{k}, \mathbf{w}\right),
$$

and we have $F_{l} E_{k} f=\left(\Pi_{2}^{\prime}\right)_{!}\left(\Pi_{1}^{*} f\right)$.

Let us introduce a subset $\mathfrak{S}=\left\{G_{\mathbf{v}}^{\mathbb{C}}\left(B, 0, j, C^{1}, C^{2}\right) \in \mathfrak{P} \mid C^{1}=C^{2}\right\}$ of $\mathfrak{P}$. When $k \neq l$, we have $\mathfrak{S}=\varnothing$. If $G_{\mathrm{v}}^{\mathbb{C}}\left(B, 0, j, C^{1}, C^{2}\right) \in \mathfrak{P} \backslash \mathfrak{S}, C^{1} \cap C^{2}$ is a collection of subspaces having the dimension $\mathbf{v}-\mathbf{e}^{k}-\mathbf{e}^{l}$. Consider the pair of restrictions of $(B, 0, j)$ to $C^{1}$ and $C^{1} \cap C^{2}$. Then the $G_{\mathbf{v}-\mathbf{e}^{-}}^{\mathbb{C}}$-orbit through it is a point in $\mathfrak{F}\left(\mathbf{v}-\mathbf{e}^{l}, \mathbf{w} ; \mathbf{e}^{k}\right)$. Similarly, we can associate a point in $\mathfrak{F}\left(\mathbf{v}-\mathbf{e}^{k}, \mathbf{w} ; \mathbf{e}^{l}\right)$. Thus there exists a continuous map

$$
\Phi: \mathfrak{P} \backslash \mathfrak{S} \rightarrow \mathfrak{P}^{\prime} .
$$

We have $\Pi_{1}^{\prime} \circ \Phi=\Pi_{1}, \Pi_{2}^{\prime} \circ \Phi=\Pi_{2}$.

Let $\mathfrak{S}^{\prime}=\left\{\left(x^{1}, x^{2}\right) \in \mathfrak{P}^{\prime} \mid x^{1}=x^{2}\right\}$. We have $\mathfrak{S}^{\prime}=\varnothing$, if $k \neq l$. We shall show that $\Phi$ gives a homeomorphism between $\mathfrak{P} \backslash \mathfrak{S}$ and $\mathfrak{P}^{\prime} \backslash \mathfrak{S}^{\prime}$ by constructing the inverse of $\Phi$.

Suppose $\left(x^{1}, x^{2}\right) \in \mathfrak{P}^{\prime}$ is given. Take a collection of vector spaces $V^{\prime}=\left(V_{m}^{\prime}\right)$ with the dimension $\mathbf{v}-\mathbf{e}^{k}-\mathbf{e}^{l}$, and a representative $\left(B^{\prime}, 0, j^{\prime}\right)$ of $\pi_{1}^{1}\left(x^{1}\right)=\pi_{1}^{2}\left(x^{2}\right)$. Take a collection of vector spaces $E^{k}$ with the dimension $\mathbf{e}^{k}$, i.e., $E_{m}^{k}=0$ if $m \neq k$ and $E_{k}^{k}=\mathbb{C}$. Similarly take $E^{l}$. Then define

$$
C^{1}=V^{\prime} \oplus E^{k}, \quad C^{2}=V^{\prime} \oplus E^{l}, \quad V=V^{\prime} \oplus E^{k} \oplus E^{l}
$$

(take the direct sum at each vertex). Take a representative $\left(B^{1}, 0, j^{1}, V^{\prime}\right)$ (resp. $\left.\left(B^{2}, 0, j^{2}, V^{\prime}\right)\right)$ of $x^{1}$ (resp. $\left.x^{2}\right)$ so that its restriction to $V^{\prime}$ is equal to $\left(B^{\prime}, 0, j^{\prime}\right)$. Then $\left(B^{1}, 0, j^{1}\right)$ and $\left(B^{2}, 0, j^{2}\right)$ define a datum $(B, 0, j)$ on $V$ by

$$
\begin{aligned}
& B\left(v^{\prime} \oplus x \oplus y\right) \stackrel{\text { def. }}{=} B^{1}\left(v^{\prime} \oplus x\right)+B^{2}(0 \oplus y), \\
& j\left(v^{\prime} \oplus x \oplus y\right) \stackrel{\text { def. }}{=} j^{1}\left(v^{\prime} \oplus x\right)+j^{2}(0 \oplus y)
\end{aligned}
$$

where $v^{\prime} \oplus x \oplus y \in V^{\prime} \oplus E^{k} \oplus E^{l}=V$, and $C^{1}, C^{2}$ are considered as subspaces of $V$, and $v^{\prime} \oplus x$ (resp. $\left.0 \oplus y\right)$ is considered as an element of $C^{1}$ (resp. $C^{2}$ ). Then $(B, 0, j)$ satisfies the complex ADHM equation. We want to define the inverse of 
$\Phi$ as $\mathfrak{P}^{\prime} \backslash \mathfrak{\Xi}^{\prime} \ni\left(x^{1}, x^{2}\right) \mapsto G_{v}^{\mathbb{C}}\left(B, 0, j, C^{1}, C^{2}\right) \in \mathfrak{P}$. We show that $(B, 0, j)$ satisfies the condition (3.5) (1) if and only if $\left(x^{1}, x^{2}\right) \notin \widetilde{S}^{\prime}$.

If $k=l$ and $x^{1}=x^{2}$, take representatives so that $\left(B^{1}, 0, j^{1}\right)=\left(B^{2}, 0, j^{2}\right)$. Then $\mathbb{C}(0 \oplus 1 \oplus-1) \subset V^{\prime} \oplus E^{k} \oplus E^{k}=V$ is $B$-stable and in the kernel of $j$. Hence $(B, 0, j)$ does not satisfy $(3.5)(1)$.

Suppose there exists a nontrivial $B$-stable collection $S=\left(S_{m}\right)_{m}$ of subspaces in $V_{m}$ such that $j(S)=0$. Since the restriction $\left(B^{1}, 0, j^{1}\right)$ satisfies $(3.5)(1)$, the intersection $S \cap C^{1}$ is zero. In particular, $S$ has nonzero components only on the vertex $k$. Similarly, we have $S \cap C^{2}=0$ and $S_{m} \neq 0$ only if $m=l$. Hence $S=0$ if $k \neq l$. Therefore we may assume $k=l$, and $S_{m}=0$ for all $m \neq k$. Write a nonzero element in $S_{k}$ as $v^{\prime} \oplus a \oplus b$ according to the decomposition $V_{k}=V_{k}^{\prime} \oplus \mathbb{C} \oplus \mathbb{C}$. Since $S \cap C^{1}=0, S \cap C^{2}=0$, we must have $a \neq 0, b \neq 0$. Since $S$ is $B$-stable, we have $B_{h}^{1}\left(v^{\prime} \oplus a\right)+B_{h}^{2}(0 \oplus b)=0$ for all $h$ with out $(h)=k$. Define a map $g: C_{k}^{2} \rightarrow C_{k}^{1}$ by

$$
g=\left(\begin{array}{ll}
1 & -v^{\prime} / b \\
0 & -a / b
\end{array}\right): C_{k}^{2}=V_{k}^{\prime} \oplus \mathbb{C} \rightarrow C_{k}^{1}=V_{k}^{\prime} \oplus \mathbb{C} .
$$

It is invertible and satisfies $B_{h}^{2}=B_{h}^{1} g$. This shows $\left(B^{1}, 0, j^{1}, V^{\prime}\right)$ and $\left(B^{2}, 0, j^{2}, V^{\prime}\right)$

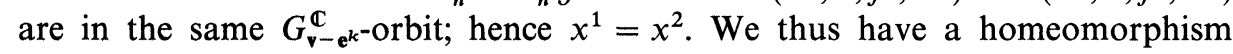
between $\mathfrak{P} \backslash \mathfrak{S}$ and $\mathfrak{P}^{\prime} \backslash \mathfrak{S}^{\prime}$.

Now the additivity of Euler characteristics implies

$$
\begin{aligned}
\left(E_{k} F_{l}\right. & \left.f-F_{l} E_{k} f\right)(x) \\
\quad= & \left.\sum_{a \in \mathbb{Q}} a\left(\chi\left(\left(\Pi_{1}^{*} f\right)^{-1}(a) \cap \Pi_{2}^{-1}(x) \cap \Im\right)-\chi\left(\Pi_{1}^{*} f\right)^{-1}(a) \cap \Pi_{2}^{\prime-1}(x) \cap \Xi^{\prime}\right)\right)
\end{aligned}
$$

for $f \in M\left(\mathfrak{L}\left(\mathbf{v}-\mathbf{e}^{l}, \mathbf{w}\right)\right)$ and $x \in \mathfrak{Q}\left(\mathbf{v}-\mathbf{e}^{k}, \mathbf{w}\right)$. In particular, we have $E_{k} F_{l}=F_{l} E_{k}$ if $k \neq l$. Henceforth we may assume $k=l$. Since we have $\Pi_{1}\left(\Pi_{2}^{-1}(x) \cap \Im\right)=x$, $\Pi_{1}^{\prime}\left(\Pi_{2}^{\prime-1}(x) \cap \mathfrak{S}^{\prime}\right)=x$, we have

$$
\left(E_{k} F_{l} f-F_{l} E_{k} f\right)(x)=\left(\chi\left(\Pi_{2}^{-1}(x) \cap \Im\right)-\chi\left(\Pi_{2}^{\prime-1}(x) \cap \Im^{\prime}\right)\right) f(x) .
$$

By Lemma 10.10, we have

$$
\chi\left(\Pi_{2}^{-1}(x) \cap \mathfrak{S}\right)-\chi\left(\Pi_{2}^{\prime-1}(x) \cap \mathfrak{S}^{\prime}\right)=\operatorname{dim} W_{k}+\sum_{l} a_{k l} \operatorname{dim} V_{l}-2\left(\operatorname{dim} V_{k}-1\right) .
$$

This shows (10.1.c).

The relations (10.1.a) and (10.1.b) are obviously satisfied, so we have the following.

Proposition 10.12. $\bigoplus_{\mathbf{v}} M(\mathfrak{L}(\mathbf{v}, \mathbf{w}))$ is a representation space of $u$.

Let $x$ be the constant function on $\mathfrak{S}(0, \mathbf{w})$ with value 1 . Let

$$
\begin{gathered}
L(\mathbf{w}) \stackrel{\text { def. }}{=} u^{-} \cdot x \subset \bigoplus_{\mathbf{v}} M(\mathfrak{L}(\mathbf{v}, \mathbf{w})), \\
L(\mathbf{v}, \mathbf{w}) \stackrel{\text { def. }}{=} M(\mathfrak{L}(\mathbf{v}, \mathbf{w})) \cap L(\mathbf{w}) .
\end{gathered}
$$


LEMMA 10.13. We have

$$
F_{k}^{w_{k}+1} x=0 \quad \text { for } k=1, \ldots, n
$$

where $\mathbf{w}={ }^{t}\left(\operatorname{dim} W_{1}, \ldots, \operatorname{dim} W_{n}\right)$.

Proof. Let $\mathbf{v}$ be such that $v_{k}=w_{k}+1, v_{l}=0$ for $l \neq k$. Then $j_{k}: V_{k} \rightarrow W_{k}$ is never injective. By Proposition 3.5 we have $\Lambda(\mathbf{v}, \mathbf{w}) \cap H_{\zeta}^{\mathrm{s}}=\varnothing$. Hence we have $M(\mathfrak{L}(\mathbf{v}, \mathbf{w}))=0$ and the assertion.

Therefore we get the main result in this section.

THEOREM 10.14. The operators $E_{k}, F_{k}, H_{k}$ on $L(\mathbf{w})$ give the irreducible highestweight integrable representation of the Kac-Moody algebra associated with the generalized Cartan matrix $\mathbf{C}$ with the highest weight $\mathbf{w}$. Each summand of the decomposition $L(\mathbf{w})=\bigoplus_{\mathbf{v}} L(\mathbf{v}, \mathbf{w})$ is a weight space with the weight $\mathbf{w}-\mathbf{C v}$.

Let $Y \in \operatorname{Irr} \mathfrak{L}(\mathbf{v}, \mathbf{w})$ be an irreducible component of $\mathfrak{L}(\mathbf{v}, \mathbf{w})$. Following [L4, 3.8] we define a linear function $T_{Y}: V(\mathbf{v}, \mathbf{w}) \rightarrow \mathbb{Q}$ for $Y \in \operatorname{Irr} \mathfrak{L}(\mathbf{v}, \mathbf{w})$; it associates to a constructible function $f \in L(\mathbf{v}, \mathbf{w})$ the (constant) value of $f$ on a suitable open dense subset of $Y$. Since $L(\mathbf{v}, \mathbf{w})$ is finite-dimensional, we can take such an open set on which any $f \in L(\mathbf{v}, \mathbf{w})$ is constant. Thus we have a linear map

$$
\Phi: L(\mathbf{v}, \mathbf{w}) \rightarrow \mathbb{Q}^{\operatorname{Irr} \mathfrak{Q}(\mathbf{v}, \mathbf{w})},
$$

where $\mathbb{Q}^{\operatorname{Irr} \mathfrak{Q}(\mathbf{v}, \mathbf{w})}$ is the $\mathbb{Q}$-vector space of $\mathbb{Q}$-valued functions on $\operatorname{Irr} \mathfrak{L}(\mathbf{v}, \mathbf{w})$, and is isomorphic to the cohomology group $H^{k}\left(\mathfrak{M}_{\zeta}(\mathbf{v}, \mathbf{w}) ; \mathbb{Q}\right)$ with $k=\operatorname{dim}_{\mathbb{C}} \mathfrak{M}_{\zeta}(\mathbf{v}, \mathbf{w})$ by (5.5).

The following is proved in [L4, 4.16].

Proposition 10.15. (1) The linear map $\Phi$ is surjective; for any $Y \in \operatorname{Irr} \mathfrak{I}(\mathbf{v}, \mathbf{w})$, there is a function $f_{Y} \in L(\mathbf{v}, \mathbf{w})$ such that for some open dense subset $O$ of $Y$ we have $\left.f\right|_{o}=1$ and such that for some closed $G_{\mathbf{v}}^{\mathbb{C}}$-invariant subset $H \subset \mathfrak{L}(\mathbf{v}, \mathbf{w})$ of dimension $<\operatorname{dim} \mathfrak{I}(\mathbf{v}, \mathbf{w})$ we have $f=0$ outside $Y \cup H$.

(2) When the underlying graph is of type $A, D, E$, or affine type, $\Phi$ is an isomorphism.

Lusztig treated only $\Lambda(\mathbf{v}, 0)$ instead of $\mathfrak{L}(\mathbf{v}, \mathbf{w})$. Since $\pi: H_{\zeta}^{\mathrm{s}} \cap \Lambda(\mathbf{v}, \mathbf{w}) \rightarrow \mathfrak{L}(\mathbf{v}, \mathbf{w})$ is a principal $G_{\mathbf{v}}^{\mathbb{C}}$-bundle, the irreducible components of $\mathfrak{L}(\mathbf{v}, \mathbf{w})$ are a projection of irreducible components of $H_{\zeta}^{\mathrm{s}} \cap \Lambda(\mathbf{v}, \mathbf{w})$. Since $\Lambda(\mathbf{v}, \mathbf{w}) \backslash H_{\zeta}^{\mathrm{s}}$ is a subvariety in $\Lambda(\mathbf{v}, \mathbf{w})=\Lambda(\mathbf{v}, 0) \times \operatorname{Hom}_{k}\left(V_{k}, W_{k}\right)$, the irreuducible components of $H_{\zeta}^{\mathrm{s}} \cap \Lambda(\mathbf{v}, \mathbf{w})$ can be identified with irreducible components of $\Lambda(v, 0)$. However, notice that an irreducible component $Y$ of $\Lambda(\mathbf{v}, \mathbf{w})$ may be entirely contained in the complement of $H_{\zeta}^{\mathrm{s}}$. Hence we only have $\operatorname{Irr} \mathfrak{L}(\mathbf{v}, \mathbf{w}) \subset \operatorname{Irr} \Lambda(\mathbf{v}, 0)$.

THEOREM 10.16. When the underlying graph is of type $A, D, E$, or affine, the middle cohomology group $H^{k}\left(\mathfrak{M}_{\zeta}(\mathbf{v}, \mathbf{w}) ; \mathbb{Q}\right)\left(k=\operatorname{dim}_{\mathbb{C}} \mathfrak{M}_{\zeta}(\mathbf{v}, \mathbf{w})\right)$ of the moduli space 
is isomorphic to $L(\mathbf{v}, \mathbf{w})$, and hence to the weight space of the irreducible highestweight integrable representation of the corresponding Kac-Moody algebra, where the highest weight is $\mathbf{w}$, and the weight is $\mathbf{w}-\mathbf{C v}$.

The middle homology group $H_{k}\left(\mathfrak{M}_{\zeta}(\mathbf{v}, \mathbf{w}) ; \mathbb{Q}\right)$ has a basis parametrized by $\operatorname{Irr} \mathfrak{L}(\mathbf{v}, \mathbf{w})$. Thus the dual basis gives a basis on the weight space $L(\mathbf{v}, \mathbf{w})$. By its construction, the following is clear.

THEOREM 10.17. Suppose the underlying graph is of type A, D, E, or affine. There exists a basis $\mathbf{B}$ of $u^{-}$with the following property: for the irreducible highestweight integrable representation $L$ with a highest-weight vector $x$, let $\pi: u^{-} \rightarrow L$; $\alpha \rightarrow \alpha x$. Then $\mathbf{B} \backslash\left(\mathbf{B} \cap \pi^{-1}(0)\right)$ gives a basis of $L$ under the map $\pi$.

The corresponding property for the "canonical bases" of quantized enveloping algebras are obtained in $[\mathrm{L} 2,8.10],[\mathrm{L} 3,11.10]$.

11. Representations of quantized enveloping algebras. In this section we assume that the underlying graph is of Dynkin type. Let $\mathbf{U}$ be the quantized universal enveloping algebra associated by Drinfeld and Jimbo to a symmetric positive definite Cartan matrix $\left(c_{k l}\right)_{1 \leqslant k, l \leqslant n}$. Let $v$ be an indeterminate. Let us introduce $v$-integers

$$
[n]=\frac{v^{n}-v^{-n}}{v-v^{-1}}, \quad[n]_{!}=[n][n-1] \cdots[1], \quad\left[\begin{array}{c}
m \\
n
\end{array}\right]=\frac{[m]_{!}}{[n]_{!}[m-n]_{!}}
$$

Then $\mathbf{U}$ is the $\mathbb{Q}(v)$-algebra with generators $E_{k}, F_{k}, K_{k}, K_{k}^{-1}(1 \leqslant k \leqslant n)$ with relations (we use the same symbol in $\S 10$, hoping this causes no confusion)

$$
\begin{gathered}
K_{k} K_{l}=K_{l} K_{k}, \quad K_{k} K_{k}^{-1}=1, \\
K_{k} E_{l}=v^{c_{k l}} E_{l} K_{k}, \quad K_{k} F_{l}=v^{-c_{k l}} F_{l} K_{k}, \\
E_{k} F_{l}-F_{l} E_{k}=\delta_{k l} \frac{K_{k}-K_{k}^{-1}}{v-v^{-1}}, \\
\sum_{p=0}^{1-c_{k l}}(-1)^{p}\left[\begin{array}{c}
1-c_{k l} \\
p
\end{array}\right] E_{k}^{p} E_{l} E_{k}^{1-c_{k l}-p}=0 \quad(k \neq l), \\
\sum_{p=0}^{1-c_{k l}}(-1)^{p}\left[\begin{array}{c}
1-c_{k l} \\
p
\end{array}\right] F_{k}^{p} F_{l} F_{k}^{1-c_{k l}-p}=0 \quad(k \neq l) .
\end{gathered}
$$

Let $\mathbf{U}^{-}$be the $\mathbb{Q}(v)$-subalgebra of $\mathbf{U}$ generated by the elements $F_{k}(1 \leqslant k \leqslant n)$.

Let $\mathbf{w}=\left(w_{1}, \ldots, w_{n}\right) \in\left(\mathbb{Z}_{\geqslant 0}\right)^{n}$. Let $L(\mathbf{w})$ be the irreducible integrable highestweight $\mathbf{U}$-module with highest weight $\mathbf{w}$. It has a nonzero vector $x$ with the following properties: 
(1) $E_{k} x=0, K_{k} x=v^{w_{k} x}$ for all $k$,

(2) the map $\pi$ : $\mathbf{U}^{-} \rightarrow L(\mathbf{w}) ; \alpha \mapsto \alpha x$ is surjective and its kernel is the left ideal in $\mathbf{U}^{-}$generated by $F_{k}^{w_{k}+1}(1 \leqslant k \leqslant n)$.

It is also known that every finite-dimensional representation is completely reducible and the irreducible one is obtained as above for some w [L1], [Ro].

Choose an orientation and consider the vector spaces given in (2.1). To emphasize the dimensions, we use the notation $\mathbf{M}_{\boldsymbol{\Omega}}(\mathbf{v}, \mathbf{w}), \mathbf{M}_{\bar{\Omega}}(\mathbf{v}, \mathbf{w})$. Let $\mathbf{v}, \mathbf{v}^{\prime}, \mathbf{v}^{\prime \prime}$ be as before, and consider the diagram

$$
\mathbf{M}_{\Omega}\left(\mathbf{v}^{\prime \prime}, 0\right) \times \mathbf{M}_{\Omega}\left(\mathbf{v}^{\prime}, 0\right) \stackrel{p_{1}}{\leftarrow} \tilde{\mathbf{E}}\left(\mathbf{v}, 0 ; \mathbf{v}^{\prime \prime}\right) \stackrel{p_{2}}{\rightarrow} \mathbf{E}\left(\mathbf{v}, 0 ; \mathbf{v}^{\prime \prime}\right) \stackrel{p_{3}}{\rightarrow} \mathbf{M}_{\Omega}(\mathbf{v}, 0)
$$

where the notations are as follows.

A point of $\mathbf{E}\left(\mathbf{v}, 0 ; \mathbf{v}^{\prime \prime}\right)$ is a point $B=\left(B_{h}\right) \in \mathbf{M}_{\Omega}(\mathbf{v}, 0)$ together with a collection of subspaces $C_{k}$ of $V_{k}$ for each vertex $k$ such that $C=\left(C_{k}\right)_{k}$ is $B$-stable and has the dimension $\mathbf{v}^{\prime}=\mathbf{v}-\mathbf{v}^{\prime \prime}$. A point of $\tilde{\mathbf{E}}\left(\mathbf{v}, 0 ; \mathbf{v}^{\prime \prime}\right)$ is a point $(B, C)$ of $\mathbf{E}\left(\mathbf{v}, 0 ; \mathbf{v}^{\prime \prime}\right)$ together with a collection of isomorphisms $R_{k}^{\prime \prime}: V_{k}^{\prime \prime} \cong C_{k}, R_{k}^{\prime}: V_{k}^{\prime} \cong V_{k} / C_{k}$ for each vertex $k$. Let us define $p_{2}\left(B, C, R^{\prime}, R^{\prime \prime}\right)=(B, C), p_{3}(B, C)=B$, and $p_{1}\left(B, C, R^{\prime}, R^{\prime \prime}\right)=$ $\left(B^{\prime \prime}, B^{\prime}\right)$ is defined as in $\S 10$. We write $\mathscr{Q}_{\mathbf{v}, \Omega}$ for the derived category of bounded complexes of $G_{\mathbf{v}}^{\mathbb{C}}$-equivariant sheaves on $\mathbf{M}_{\Omega}(\mathbf{v}, 0)$. (For a general graph, one must restrict a certain class of complexes of equivariant sheaves. See [L3] for details.)

Let $L^{\prime} \in \mathscr{Q}_{\mathbf{v}^{\prime}, \boldsymbol{\Omega}}, L^{\prime \prime} \in \mathscr{Q}_{\mathbf{v}^{\prime \prime}, \boldsymbol{\Omega}}$. We associated $L^{\text {def. }}=L^{\prime} * L^{\prime \prime} \in \mathscr{Q}_{\mathbf{v}, \boldsymbol{\Omega}}$ as follows: Consider the exterior tensor product $L_{1} \stackrel{\text { def. }}{=} L^{\prime} \otimes L^{\prime \prime}$ on $\mathbf{M}_{\Omega}\left(\mathbf{v}^{\prime}, 0\right) \times \mathbf{M}_{\Omega}\left(\mathbf{v}^{\prime \prime}, 0\right)$. Then there is a unique complex $L_{3}$ such that $p_{2}^{*}\left(L_{3}\right) \cong p_{1}^{*}\left(L_{1}\right)$. We define $L^{\prime} * L^{\prime \prime} \stackrel{\text { def. }}{=}\left(p_{3}\right)_{!} L_{3}$.

Let $\mathscr{K}_{\mathbf{v}, \Omega}$ be the Grothendieck group of $\mathscr{Q}_{\mathbf{v}, \Omega}$. It can be considered as a module over $\mathbb{Z}\left[v, v^{-1}\right]$ by defining $v(L)=L[1], v^{-1}=L[-1]$. The operation $*$ makes $\mathscr{K}_{\Omega} \stackrel{\text { def. }}{=} \bigoplus_{v} \mathscr{K}_{\mathbf{v}, \Omega}$ into an associative graded $\mathbb{Z}\left[v, v^{-1}\right]$-algebra. We define a new associative $\mathbb{Z}\left[v, v^{-1}\right]$-algebra structure $\circ$ by

$$
\left(a^{\prime}, a^{\prime \prime}\right) \mapsto a^{\prime} \circ a^{\prime \prime}=v^{m_{\Omega}\left(\mathbf{v}^{\prime}, \mathbf{v}^{\prime \prime}\right)} a^{\prime} * a^{\prime \prime}, \quad \text { for } a^{\prime} \in \mathscr{K}_{\mathbf{v}^{\prime}, \Omega}, a^{\prime \prime} \in \mathscr{K}_{\mathbf{v}^{\prime \prime}, \Omega},
$$

where

$$
m_{\Omega}\left(\mathbf{v}^{\prime}, \mathbf{v}^{\prime \prime}\right) \stackrel{\text { def. }}{=} \sum_{h \in \Omega} v_{\mathrm{out}(h)}^{\prime} v_{\mathrm{in}(h)}^{\prime \prime}+\sum_{m} v_{m}^{\prime} v_{m}^{\prime \prime}
$$

For each vertex $k$ consider $\mathbf{v}$ such that $v_{k}=1$ and $v_{l}=0$ for $k \neq l$. Let $F_{k}^{(1)}$ be the constant complex $\mathbb{Q}_{\mathbf{M}_{\Omega}(\mathbf{v}, 0)}$ on $\mathbf{M}_{\Omega}(\mathbf{v}, 0)=\{0\}$. Now the result in [L3, 10.17] (see also [Ri], [L2, 9.4]) can be stated as the following.

THEOREM 11.3. There is a unique $\mathbb{Q}(v)$-algebra isomorphism $\lambda_{\Omega}: \mathbf{U}^{-} \rightarrow \mathscr{K}_{\Omega} \otimes$ $\mathbb{Q}(v)$ such that $\lambda_{\Omega}\left(F_{k}\right)=F_{k}^{(1)}$.

For a complex $L$ on a smooth algebraic variety $X$, we denote by $S S(L)$ the microsupport of $L$, which is known to be a closed Lagrangian subvariety of $T^{*} X$ (see [KS]). If $p: X \rightarrow X^{\prime}$ is a map, the transpose of its differential is $T^{*} p$ : 
$p^{*} T^{*} X^{\prime} \rightarrow T^{*} X$, where $p^{*} T^{*} X^{\prime}$ is the pullback of $T^{*} X^{\prime}$ by $p$. Let $p_{\pi}: p^{*} T^{*} X^{\prime} \rightarrow$ $T^{*} X^{\prime}$ be the natural map. Then we have the following functorial properties of the microsupport:

(11.4.a) $S S(L[1])=S S(L)[\mathrm{KS}, 5.1 .3]$;

(11.4.b) if $L$ (resp. $M)$ is a complex on $X$ (resp. $Y$ ), then $S S(L \otimes M) \subset S S(L) \times$ $S S(M)[\mathrm{KS}, 5.4 .1]$

(11.4.c) if $L$ is a complex on $X$ and $p$ is proper, $S S\left(p_{!} L\right) \subset p_{\pi}\left(T^{*} p^{-1}(S S(L))\right.$ [KS, 5.4.4];

(11.4.d) if $L$ is a complex on $X^{\prime}$ and $p$ is smooth, $S S\left(p^{*} L\right)=T^{*} p\left(p_{\pi}^{-1}(S S(L))\right)$ [KS, 5.4.5].

We study $T^{*}$ 's of maps in the diagram (11.2). The cotangent bundle $T^{*} \mathbf{M}_{\Omega}(\mathbf{v}, 0)$ is identified with $\mathbf{M}(\mathbf{v}, 0)$ via the symplectic form $\omega_{\mathbb{C}}$ (see $\left.\S 2\right)$. The cotangent space at $(B, C)$ of $\mathbf{E}\left(\mathbf{v}, 0 ; \mathbf{v}^{\prime \prime}\right)$ is the cokernel of the map

$$
\Xi: \bigoplus_{h \in \bar{\Omega}} \operatorname{Hom}\left(V_{\text {out }(h)} / C_{\text {out }(h)}, C_{\text {in }(h)}\right) \rightarrow \mathbf{M}_{\bar{\Omega}}(\mathbf{v}, 0) \oplus\left(\bigoplus_{k} \operatorname{Hom}\left(V_{k} / C_{k}, C_{k}\right)\right)
$$

given by

$$
\Xi(D)=\left(i_{\mathrm{in}(h)} D_{h} p_{\mathrm{out}(h)}, \sum_{h \in \bar{\Omega}: \operatorname{in}(h)=k} D_{h} B_{\bar{h}}-\sum_{h \in \Omega: \operatorname{in}(h)=k} B_{h} D_{\bar{h}}\right),
$$

where $i_{\mathrm{in}(h)}: C_{\mathrm{in}(h)} \rightarrow V_{\mathrm{in}(h)}$ is the inclusion map and $p_{\mathrm{out}(h)}: V_{\mathrm{out}(h)} \rightarrow V_{\mathrm{out}(h)} / C_{\mathrm{out}(h)}$ is the projection map. In the second term of the right-hand side, we regard $B_{\bar{h}}$ (resp. $B_{h}$ ) as a map $V_{\mathrm{in}(h)} / C_{\mathrm{in}(h)} \rightarrow V_{\text {out }(h)} / C_{\text {out }(h)}\left(\right.$ resp. $\left.C_{\text {out }(h)} \rightarrow C_{\mathrm{in}(h)}\right)$. The linear map $T^{*} p_{3}: T_{B}^{*} \mathbf{M}_{\Omega}(\mathbf{v}, 0) \cong \mathbf{M}_{\bar{\Omega}}(\mathbf{v}, 0) \rightarrow T_{(B, C)}^{*} \mathbf{E}\left(\mathbf{v}, \mathbf{w} ; \mathbf{v}^{\prime \prime}\right)$ is given by $X \mapsto(X, 0) \bmod \operatorname{Im} \Xi$.

The cotangent space at $\left(B, C, R^{\prime}, R^{\prime \prime}\right)$ of $\tilde{\mathbf{E}}\left(\mathbf{v}, \mathbf{w} ; \mathbf{v}^{\prime \prime}\right)$ is the cokernel of the map

$$
\begin{aligned}
\Lambda: & \bigoplus_{h \in \bar{\Omega}} \operatorname{Hom}\left(V_{\text {out }(h)} / C_{\text {out }(h)}, C_{\text {in }(h)}\right) \oplus \underset{k}{\bigoplus}\left(\operatorname{Hom}\left(V_{k} / C_{k}, V_{k}^{\prime \prime}\right) \oplus \operatorname{Hom}\left(V_{k}^{\prime}, C_{k}\right)\right) \\
& \rightarrow \mathbf{M}_{\bar{\Omega}}(\mathbf{v}, 0) \oplus \underset{k}{ }\left(\operatorname{Hom}\left(V_{k} / C_{k}, C_{k}\right) \oplus \operatorname{Hom}\left(V_{k}, V_{k}^{\prime \prime}\right) \oplus \operatorname{Hom}\left(V_{k}^{\prime}, V_{k}\right)\right)
\end{aligned}
$$

given by

$$
\begin{aligned}
\Lambda(D, E, F)= & \left(i_{\operatorname{in}(h)} D_{h} p_{\text {out }(h)}, \sum_{h \in \bar{\Omega}: \operatorname{in}(h)=k} D_{h} B_{\bar{h}}\right. \\
& \left.-\sum_{h \in \Omega: \operatorname{in}(h)=k} B_{h} D_{\bar{h}}-R_{k}^{\prime \prime} E_{k}+F_{k} R_{k}^{\prime-1}, E_{k} p_{k}, i_{k} F_{k}\right) .
\end{aligned}
$$

Then $T^{*} p_{2}: T_{(B, C)}^{*} \mathbf{E}^{\prime \prime} \rightarrow T_{\left(B, C, R^{\prime}, R^{\prime \prime}\right)}^{*} \mathbf{E}^{\prime}$ is given by

$$
(X, Y) \bmod \operatorname{Im} \Xi \mapsto(X, Y, 0,0) \bmod \operatorname{Im} \Lambda .
$$


Finally, $T^{*} p_{1}: T_{B^{\prime}}^{*} \mathbf{M}_{\Omega}\left(\mathbf{v}^{\prime}, 0\right) \oplus T_{B^{\prime \prime}}^{*} \mathbf{M}_{\mathbf{\Omega}}\left(\mathbf{v}^{\prime \prime}, 0\right) \rightarrow T_{\left(B, C, \boldsymbol{R}^{\prime}, \boldsymbol{R}^{\prime \prime}\right)}^{*} \mathbf{E}^{\prime}$ is given by

$$
\begin{aligned}
\left(X^{\prime}, X^{\prime \prime}\right) & \mapsto(X, 0, Z, W) \bmod \operatorname{Im} \Lambda ; \\
X_{h} & =\tilde{R}_{\mathrm{in}(h)}^{\prime} X_{h}^{\prime} R_{\mathrm{out}(h)}^{\prime-1} p_{\mathrm{out}(h)}+i_{\mathrm{in}(h)} R_{\mathrm{in}(h)}^{\prime \prime} X_{h}^{\prime \prime} R_{\mathrm{out}(h)}^{\prime \prime-1} \tilde{\pi}_{\mathrm{out}(h)} \\
Z_{k} & =\sum_{h \in \bar{\Omega}: \operatorname{in}(h)=k} X_{h}^{\prime \prime} R_{\mathrm{out}(h)}^{\prime \prime-1} \tilde{\pi}_{\mathrm{out}(h)} B_{\bar{h}}-\sum_{h \in \Omega: \operatorname{in}(h)=k} B_{h}^{\prime \prime} X_{\bar{h}}^{\prime \prime} R_{k}^{\prime \prime-1} \tilde{\pi}_{k} \\
W_{k} & =\sum_{h \in \bar{\Omega}: \operatorname{in}(h)=k} B_{h} \tilde{R}_{\mathrm{out}(h)}^{\prime} X_{\bar{h}}^{\prime}-\sum_{h \in \Omega: \operatorname{in}(h)=k} \tilde{R}_{k}^{\prime} X_{h}^{\prime} B_{\bar{h}}^{\prime},
\end{aligned}
$$

where $\tilde{R}_{k}^{\prime}: V_{k}^{\prime} \rightarrow V_{k}$ is a lift of $R_{k}^{\prime}$, and $\tilde{\pi}_{k}: V_{k} \rightarrow C_{k}$ is a projection onto $C_{k}$. The ambiguities coming from choices of the lift and the projection are absorbed in $\operatorname{Im} \Lambda$.

Take a vector $\mathbf{w}$ as before and let $\mathscr{K}_{\Omega}^{1}(\mathbf{w})$ be the $\mathbb{Z}\left[v, v^{-1}\right]$-submodule of $\mathscr{K}_{\Omega}$ generated by complexes $L$ in $\mathscr{Q}_{\mathrm{v}, \Omega}$ such that $S S(L) \times \bigoplus_{k} \operatorname{Hom}\left(V_{k}, W_{k}\right)$ does not intersect with $H_{\zeta}^{\text {s. }}$. Recall that $S S(L)$ is a union of irreducible components of $\Lambda(\mathbf{v}, 0)$ by $[\mathrm{L} 3,13.6]$.

LEMMA 11.5. $\quad \mathscr{K}_{\Omega}^{1}(\mathbf{w}) \otimes \mathbb{Q}(v)$ is a left ideal in $\mathscr{K}_{\Omega} \otimes \mathbb{Q}(v)$ containing $\left(F_{k}^{(1)}\right)^{w_{k}+1}$ $(1 \leqslant k \leqslant n)$.

Proof. Let $L^{\prime} \in \mathscr{Q}_{\mathbf{v}^{\prime}, \Omega}, L^{\prime \prime} \in \mathscr{Q}_{\mathbf{v}^{\prime \prime}, \Omega}$. We want to study the microsupport of $S S\left(L^{\prime} * L^{\prime \prime}\right)$. Define $L_{1}, L_{3}$ as before. Since $L^{\prime} * L^{\prime \prime}=\left(p_{3}\right)_{!} L_{3}$ and $p_{1}^{*}\left(L_{1}\right)=p_{2}^{*}\left(L_{3}\right)$, we have

$$
\begin{gathered}
S S\left(L^{\prime} * L^{\prime \prime}\right) \subset\left(p_{3}\right)_{\pi}\left(T^{*} p_{3}^{-1}\left(S S\left(L_{3}\right)\right),\right. \\
T^{*} p_{2}\left(\left(p_{2}\right)_{\pi}^{-1}\left(S S\left(L_{3}\right)\right)=T^{*} p_{1}\left(\left(p_{1}\right)_{\pi}^{-1}\left(S S\left(L_{1}\right)\right),\right.\right. \\
S S\left(L_{1}\right) \subset S S\left(L^{\prime}\right) \times S S\left(L^{\prime \prime}\right) .
\end{gathered}
$$

Take $\left(B, C, R^{\prime}, R^{\prime \prime}\right) \in \mathbf{E}^{\prime}$ and define $B^{\prime}, B^{\prime \prime}$ as before. If $X \in T_{B}^{*} \mathbf{M}_{\Omega}(\mathbf{v}, 0)$ is contained in $S S\left(L^{\prime} * L^{\prime \prime}\right)$, then the above shows

$$
\begin{gathered}
T^{*} p_{2} T^{*} p_{3}(X)=T^{*} p_{1}\left(X^{\prime}, X^{\prime \prime}\right) \\
\text { for some }\left(X^{\prime}, X^{\prime \prime}\right) \in\left(T_{B^{\prime}}^{*} \mathbf{M}\left(\mathbf{v}^{\prime}, 0\right) \cap S S\left(L^{\prime}\right)\right) \times\left(T_{B^{\prime \prime}}^{*} \mathbf{M}\left(\mathbf{v}^{\prime \prime}, 0\right) \cap S S\left(L^{\prime \prime}\right)\right)
\end{gathered}
$$

Then we have $X_{h}\left(C_{\mathrm{out}(h)}\right) \subset C_{\mathrm{in}(h)}$ and $R_{\mathrm{in}(h)}^{\prime} X_{h}^{\prime}=X_{h} R_{\mathrm{out}(h)}^{\prime}, R_{\mathrm{out}(h)}^{\prime \prime} X_{h}^{\prime \prime}=X_{h} R_{\mathrm{in}(h)}^{\prime \prime}$ for all $h \in \bar{\Omega}$. Hence Lemma 10.3 implies that $\left(B^{\prime \prime}, X^{\prime \prime}, 0, j^{\prime \prime}\right)$ satisfies the condition (3.5) (1) if $(B, X, 0, j)$ satisfies it, where $j_{k}^{\prime \prime}=j_{k} R_{k}^{\prime \prime}$. This shows $\mathscr{K}_{1, \Omega}(\mathbf{w}) \otimes \mathbb{Q}(v)$ is a left ideal in $\mathscr{K}_{\Omega} \otimes \mathbb{Q}(v)$.

The last statement $\left(F_{k}^{(1)}\right)^{w_{k}+1} \in \mathscr{K}_{\Omega}^{1}(\mathbf{w}) \otimes \mathbb{Q}(v)$ can be proved as in 10.13 . 
By this lemma, we have a surjective $\mathbf{U}^{-}$-morphism

$$
\lambda_{\Omega}^{\prime}: L(\mathbf{w}) \rightarrow \mathscr{K}_{\Omega} \otimes \mathbb{Q}(v) / \mathscr{K}_{\Omega}^{1}(\mathbf{w}) \otimes \mathbb{Q}(v) .
$$

(The same holds for arbitrary graphs, after a suitable modification of the definition of $\mathscr{K}_{\Omega}$.)

Now we use the assumption that the graph is of Dynkin type. Let $y$ be a lowest-weight vector of $L(\mathbf{w})$; that is, a vector with the following property:

$$
F_{k} y=0, \quad K_{k} y=v^{w_{k}^{\prime}} y \quad \text { for all } k,
$$

where $\mathbf{w}^{\prime}=\left(w_{1}^{\prime}, \ldots, w_{n}^{\prime}\right)$ is given by $\mathbf{w}^{\prime}=\sigma \mathbf{w}$ with the unique element $\sigma$ of the Weyl group $W$ of maximal length. The lowest vector is unique up to constant.

LEMMA 11.6. The image of $y$ by $L(\mathbf{w})$ is not 0 in $\mathscr{K}_{\Omega} \otimes \mathbb{Q}(v) / \mathscr{K}_{\Omega}^{1}(\mathbf{w}) \otimes \mathbb{Q}(v)$.

Proof. Take $\mathbf{v}^{\prime}$ so that $\mathbf{w}-\mathbf{C v}^{\prime}=\sigma \mathbf{w}$. By 10.16, we have

$$
\# \operatorname{Irr} \mathfrak{L}\left(\mathbf{v}^{\prime}, \mathbf{w}\right)=\operatorname{dim} L\left(\mathbf{v}^{\prime}, \mathbf{w}\right)=\operatorname{dim} L(0, \mathbf{w})=1 .
$$

(One can prove this by using the reflection in $\S 9: \mathfrak{M}_{\zeta}\left(\mathbf{v}^{\prime}, \mathbf{w}\right)$ is isomorphic to $\mathfrak{M}_{\sigma \zeta}(0, \mathbf{w})$, and $\mathfrak{M}_{\sigma \zeta}(0, w)$ is diffeomorphic to $\mathfrak{M}_{\zeta}(0, \mathbf{w})$. But $\mathfrak{M}_{\zeta}(0, \mathbf{w})$ is a point.) We thus have a unique irreducible component $Y \in \operatorname{Irr} \Lambda\left(\mathbf{v}^{\prime}, \mathbf{w}\right)$ such that $Y \cap H_{\zeta}^{\mathrm{s}} \neq \varnothing$ (Remark $5.11(2)$ ). By Remark $5.11(3)$, such $Y$ is the conormal bundle of a $G_{v}^{\mathbb{C}}$ orbit $c$ in $\mathbf{M}\left(\mathbf{v}^{\prime}, \mathbf{w}\right) \cap\{i=0\}$.

Suppose $\lambda_{\Omega}^{\prime}(y)=0$ which means that

$$
S S(L) \times \bigoplus_{k} \operatorname{Hom}\left(V_{k}, W_{k}\right) \subset \Lambda\left(\mathbf{v}^{\prime}, \mathbf{w}\right) \backslash H_{\zeta}^{\mathrm{s}}
$$

for any $L \in \mathscr{Q}_{\mathbf{v}^{\prime}, \Omega}$. But this contradicts the above observation. The microsupport of the constant sheaf on the orbit $c$ contains its conormal bundle.

Since $\mathscr{K}_{\Omega} \otimes \mathbb{Q}(v) / \mathscr{K}_{\Omega}^{1}(\mathbf{w}) \otimes \mathbb{Q}(v)$ is a $\mathbf{U}^{-}$-module containing both highest and lowest vectors, we have the following result.

THEOREM 11.7. The map $\lambda_{\Omega}^{\prime}$ is an isomorphism between the highest-weight module $L(\mathbf{w})$ and $\mathscr{K}_{\Omega} \otimes \mathbb{Q}(v) / \mathscr{K}_{\Omega}^{1}(\mathbf{w}) \otimes \mathbb{Q}(v)$.

For a $G_{\mathbf{v}}^{\mathbb{C}}$-orbit $\mathcal{O}_{c}$ in $\mathbf{M}_{\Omega}(\mathbf{v}, 0)$, let $P_{c}$ be the simple perverse sheaf whose support is the closure of $\mathcal{O}_{c}$ and whose restriction to $\mathcal{O}_{c}$ is the constant complex (up to shift). Then, by [L2], B $\stackrel{\text { def. }}{=}\left\{P_{c}\right\}_{c, v}$ is a basis of $\mathscr{K}_{\Omega}$. By Remark $5.11(3)$, the set $\mathbf{B}$ can be identified with $\bigcup_{\mathbf{v}} \operatorname{Irr} \Lambda(\mathbf{v}, 0)=\bigcup_{\mathbf{v}} \operatorname{Irr} \Lambda(\mathbf{v}, \mathbf{w})$. Let $\Phi: \mathbb{Q}(v)^{\mathbf{B}} \rightarrow \mathscr{K}_{\Omega} \otimes \mathbb{Q}(v)$ be the map given by the basis B. By the property of the canonical basis B proved in $[\mathrm{L} 2,8.10]$, [L3, 11.10] (the property corresponding to 10.17), $\Phi$ induces an isomorphism $\Psi: \mathbb{Q}(v)^{\mathbf{B}_{1}} \rightarrow L(\mathbf{w})$, where $\mathbf{B}_{1} \stackrel{\text { def. }}{=}\left\{P_{c} \in \mathbf{B} \mid P_{c} \notin \mathscr{K}_{1, \Omega}(\mathbf{w})\right\}$.

The subset $\operatorname{Irr} \mathfrak{L}(\mathbf{v}, \mathbf{w}) \subset \operatorname{Irr} \Lambda(\mathbf{v}, \mathbf{w})$ consists of orbits whose conormal bundles 
intersect with $H_{\zeta}^{s}$ (see Remark 5.11 (2)). Then $\bigcup_{\mathbf{v}} \operatorname{Irr} \mathfrak{L}(\mathbf{v}, \mathbf{w})$ is contained in $\mathbf{B}_{\mathbf{1}}$. On the other hand, we have

$$
\# \mathbf{B}_{1}=\operatorname{dim}_{\mathbb{Q}(v)} L(\mathbf{w})=\# \bigcup_{\mathbf{v}} \operatorname{Irr} \mathfrak{L}(\mathbf{v}, \mathbf{w})
$$

where we used Theorem 10.16 and the fact that $\operatorname{dim} L(\mathbf{w})$ is equal to the dimension of the representation space of corresponding simple Lie algebra. Hence we have $\mathbf{B}_{1}=\bigcup_{\mathbf{v}} \operatorname{Irr} \mathfrak{L}(\mathbf{v}, \mathbf{w})$. Summarizing the above arguments, we obtain the following.

THEOREM 11.8. Let $P_{c}$ be the simple perverse sheaf whose support is the closure of $\mathcal{O}_{c}$ and whose restriction to $\mathcal{O}_{c}$ is the constant complex (up to shift). Then it is contained in the left ideal generated by $\left(F_{k}^{(1)}\right)^{w_{k}+1}$ if and only if its microsupport is contained in the complement of $H_{\zeta}^{\mathrm{s}}$. The set $\operatorname{Irr} \mathfrak{L}(\mathbf{v}, \mathbf{w})$ parametrizes the canonical basis of the weight space of $L(\mathbf{w})$ with the weight $\mathbf{w}-\mathbf{C v}$.

Remark 11.9. When $\mathbf{u}=\mathbf{w}-\mathbf{C v}=0$, we have two Weyl group representations on $H^{k}\left(\mathfrak{M}_{\zeta} ; \mathbb{Q}\right)\left(k=\operatorname{dim}_{\mathbb{C}} \mathfrak{M}_{\zeta}(\mathbf{v}, \mathbf{w})\right)$; one as given in Corollary 9.3 and one as the weight space with weight 0 . The latter representation can be "deformed" to a representation of the corresponding Braid group. It is desirable to know the relationship between the two representations.

12. Analogy with moduli spaces of Higgs bundles over Riemann surfaces. Quiver varieties have many similarities with moduli spaces of Higgs bundles over Riemann surfaces, which was introduced and studied by Hitchin [Hi]. Our study is partially motivated by his result, so we give an analogy here for the sake of the reader.

Let $\Sigma$ be a compact Riemann surface and $E$ a hermitian vector bundle over $\Sigma$. Let us define

$$
\mathscr{A} \stackrel{\text { def. }}{=}\{\text { unitary connections on } E\}, \quad \mathscr{G} \stackrel{\text { def. }}{=} \text { the gauge group of } E .
$$

Then $\mathscr{A}$ is an affine space isomorphic to $\Omega^{1}($ Endskew $(E))$, and $\mathscr{G}$ acts on $\mathscr{A}$ by pullback. The $L^{2}$-inner product on $\Omega^{1}($ Endskew $(E))$ can be considered as a Riemannian metric on the infinite-dimensional manifold $\mathscr{A}$. The Hodge star operator on $\Omega^{1}($ Endskew $(E))$ can be considered as a complex structure on $\mathscr{A}$. Hence $\mathscr{A}$ is an infinite-dimensional Kähler manifold. The $\mathscr{G}$-action preserves both the metric and the complex structure.

Via the isomorphism $\Omega^{1}(\operatorname{Endskew}(E)) \cong \Omega^{0,1}(\operatorname{End}(E))$, the holomorphic cotangent bundle of $\mathscr{A}$ can be identified with

$$
T^{*} \mathscr{A} \cong \mathscr{A} \times \Omega^{0,0}\left(K_{\Sigma} \otimes \operatorname{End}(E)\right),
$$

where $K_{\Sigma}$ is the canonical bundle of $\Sigma$ and the natural pairing is given by

$$
\int_{X} \operatorname{tr}(\alpha \wedge \beta) \quad \text { for } \alpha \in \Omega^{0,1}(\operatorname{End}(E)), \quad \beta \in \Omega^{0,0}\left(K_{\Sigma} \otimes \operatorname{End}(E)\right) .
$$


As in $\S 2, T^{*} \mathscr{A}$ is considered as a hyper-Kähler manifold. The gauge group action preserves the hyper-Kähler structure, and the corresponding moment map is given by

$$
\begin{aligned}
& \mu_{\mathbb{R}}(A, \Phi)=R_{A}+\left[\Phi \wedge \Phi^{\dagger}\right] \\
& \mu_{\mathbb{C}}(A, \Phi)=\bar{\partial}_{A} \Phi, \quad \text { for } A \in \mathscr{A}, \quad \Phi \in \Omega^{0,0}\left(K_{\Sigma} \otimes \operatorname{End}(E)\right)
\end{aligned}
$$

These equations are called self-duality equations in [Hi]. The hyper-Kähler quotient $\mu^{-1}(0) / \mathscr{G}$ naturally has a structure of a hyper-Kähler manifold. It may have singularities in general, but we disregard them here.

Now the analogy with quiver varieties is clear. The connection space $\mathscr{A}$ corresponds $\mathbf{M}_{\Omega}$, where both are flat Kähler manifolds. The cotangent bundle $T^{*} \mathscr{A}$ corresponds to $\mathbf{M}$, and the gauge group $\mathscr{G}$ to $G_{\mathbf{v}}$. The holomorphic description in $\S 3$ becomes an identification of $\mu^{-1}(0) / \mathscr{G}$ with the moduli space of stable Higgs bundles, which was proved in [Hi], [Si]. Quiver varieties have an $S^{1}$-action when the complex parameter is zero, i.e., $\zeta=\left(\zeta_{\mathbb{R}}, 0\right)$, while Hitchin defined an $S^{1}$-action by

$$
[(A, \Phi)] \mapsto[(A, t \Phi)] \quad t \in S^{1}
$$

Then he studied the topology by using the corresponding moment map as a Morse function. This was done in $\S 5$ in our case.

Both theories have many examples of moduli spaces in common. Donaldson and Kronheimer described the cotangent bundle of the generalized flag manifold as the moduli space of $S^{1}$-equivariant solutions of the self-duality equation on the 2-disk, while it also appears in our theory (see \$7).

We defined a resolution map $\pi: \mathfrak{M}_{\left(\zeta_{\mathbb{R}}, 0\right)} \rightarrow \mathfrak{M}_{0}$ in $\S 3$, and we have shown that $\mathfrak{L}=\pi^{-1}(0)$ is a Lagrangian variety, which plays a crucial role in $\S 10$. The corresponding thing is Hitchin's Hamiltonian fibration:

$$
\begin{aligned}
& p: \mu^{-1}(0) / \mathscr{G} \rightarrow \bigoplus_{i=1}^{\text {rank } E} H^{0}\left(\Sigma ; \mathcal{O}\left(K_{\Sigma}^{\otimes i}\right)\right) \\
& p([(A, \Phi)]) \stackrel{\text { def. }}{=}\left(\operatorname{tr} \Phi, \operatorname{tr} \Phi^{2}, \ldots, \operatorname{tr} \Phi^{\text {rank } E}\right) .
\end{aligned}
$$

Both $\pi$ and $p$ induce isomorphisms between global holomorphic sections. Hitchin proved that generic fibers of $p$ are abelian varieties, which are Lagrangian in $\mu^{-1}(0) / \mathscr{G}$ with respect to the holomorphic symplectic structure. The central fiber $p^{-1}(0)$ is a singular Lagrangian subvariety, and it corresponds to our Lagrangian subvariety $\mathfrak{R}$. (In fact, our argument in (5.8) gives another proof that $p^{-1}(0)$ is Lagrangian.) The Lagrangian subvariety $p^{-1}(0)$ plays an important role in the geometric Langlands correspondence (see Laumon [La]).

Lusztig's diagram (11.2) corresponds to the so-called Hecke correspondence. 
Constructions of quantized enveloping algebras should correspond to something in Hitchin's theory, or the geometric Langlands correspondence, but it is not clear, at least to the author.

\section{REFERENCES}

[At] M. F. AтזYAH, Convexity and commuting Hamiltonians, Bull. London Math. Soc. 14 (1982), 1-15.

[AB] M. F. Atiyah AND R. BotT, The Yang-Mills equations over Riemann surfaces, Philos. Trans. Roy. Soc. London Ser. A. 362 (1982), 523-615.

[ADHM] M. F. AtiYah, V. DRINFEld, N. J. Hitchin, AND Y. I. Manin, Construction of instantons, Phys. Lett. A 65 (1978), 185-187.

[Ba] S. BANDO, "Einstein-Hermitian metrics on non-compact Kähler manifolds" in Einstein Metrics and Yang-Mills Connections, ed. by T. Mabuchi and S. Mukai, Lecture Notes in Pure and Appl. Math. 145, Dekker, New York, 1993, 27-33.

[BLM] A. Beilinson, G. Lusztig, AND R. MacPherson, A geometric setting for quantum groups, Duke Math. J. 61 (1990), 655-675.

[BM] W. Borho AND R. MACPHERSON, Partial resolutions of nilpotent varieties, Astérisque 101102 (1983), 23-74.

[Bo] R. BotT, Nondegenerate critical manifolds, Ann. of Math. (2) 60 (1954), 248-261.

[Do] S. K. Donaldson, Instantons and geometric invariant theory, Comm. Math. Phys. 93 (1984), 453-460.

[DK] S. K. Donaldson AND P. B. Kronheimer, The geometry of four-manifolds, Oxford Math. Monographs, Clarendon, Oxford, 1990.

[Fr] T. Frankel, Fixed points and torsion on Kähler manifolds, Ann. of Math. (2) 70 (1959), $1-8$.

[Ga] P. GABRIEL, Unzerlegbare darstellungen I, Manuscripta Math. 6 (1972), 71-103.

[Gi] V. GinzBURG, Lagrangian construction of the enveloping algebra $\left.U(\mathfrak{s l})_{n}\right)$, C. R. Acad. Sci. Paris Sér. I Math. 312 (1991), 907-912.

[GN] T. Gocho AND H. NAKAJIMA, Einstein-Hermitian connections on hyper-Kähler quotients, J. Math. Soc. Japan 44 (1992), 43-51.

[Go] R. Gото, "On toric hyper-Kähler manifolds given by the hyper-Kähler quotient method" in Infinite Analysis, Adv. Ser. Math. Phys. 16, World Science, River Edge, New Jersey, 317-338.

[Hi] N. J. Hitchin, The self-duality equations on a Riemann surface, Proc. London Math. Soc. (3) 55 (1987), 59-126.

[HKLR] N. J. HitChIN, A. KARLHEde, U. LindsTRÖM, AND M. RoČEK, Hyperkähler metrics and supersymmetry, Comm. Math. Phys. 108 (1987), 535-589.

[Kac] V. G. KAC, Infinite Dimensional Lie Algebras, 3rd ed., Cambridge Univ. Press, Cambridge, 1990.

[KS] M. Kashiwara AND P. Schapira, Sheaves on Manifolds, Grundlehren Math. Wiss. 292, Springer-Verlag, Berlin, 1990.

[Kin] A. D. KING, Moduli of representations of finite dimensional algebras, to appear in Quart. J. Math. Oxford Ser. (2).

[Ki] F. C. KIRWAN, Cohomology of quotients in symplectic and algebraic geometry, Math. Notes 31, Princeton Univ. Press, Princeton, 1984.

[KP] H. KRAFT AND C. PROCESI, Closures of conjugacy classes of matrices are normal, Invent. Math. 53 (1979), 227-247.

[Kr1] P. B. KRONHEIMER, The construction of ALE spaces as a hyper-Kähler quotients, J. Differential Geom. 29 (1989), 665-683.

[Kr2] - Instantons and the geometry of the nilpotent variety, J. Differential Geom. 32 (1990), 473-490. 
[KN] P. B. KRONHEIMER AND H. NAKAJIMA, Yang-Mills instantons on ALE gravitational instantons, Math. Ann. 288 (1990), 263-307.

[La] G. LAUMON, Correspondence de Langlands géométrique pour les corps de fonctions, Duke Math. J. 54 (1987), 309-359.

[L1] G. LUSZTIG, Quantum deformations of certain simple modules over enveloping algebras, Adv. Math. 70 (1988), 237-249.

[L2] - Canonical bases arising from quantized enveloping algebras, J. Amer. Math. Soc. 3 (1990), 447-498.

[L3] - Quivers, perverse sheaves, and quantized enveloping algebras, J. Amer. Math. Soc. 4 (1991), 365-421.

[L4] - Affine quivers and canonical bases, Inst. Hautes Études Sci. Publ. Math. 76 (1992), 111-163.

[Mc] R. MACPHERson, Chern classes for singular varieties, Ann. of Math. (2) 100 (1974), 423432.

[Na1] H. NAKAJIMA, Moduli spaces of anti-self-dual connections on ALE gravitational instantons, Invent. Math. 102 (1990), 267-303.

[Na2] - Homology of moduli spaces of instantons on ALE spaces (I), to appear in $\mathbf{J}$. Differential Geom.

[Na3] - Resolutions of moduli spaces of ideal instantons on $\mathbb{R}^{4}$, proceedings of the 1993 Taniguchi Symposium on Low-dimensional Topology and Topological Field Theory, to appear.

[Na4] Gauge theory on resolution of simple singularities and simple Lie algebras, Internat. Math. Res. Notices 1994, 61-74.

[Nee] A. NeEman, The topology of quotient varieties, Ann. of Math. (2) 103 (1985), 419-459.

$[\mathrm{Ne}] \quad$ L. NESS, $A$ stratification of the null cone via the moment map, Amer. J. Math. 106 (1984), 1281-1325.

[Ri] C. M. Ringel, Hall algebras and quantum groups, Invent. Math. 101 (1990), 583-592.

[Ro] M. Rosso, Finite dimensional representations of the quantum analog of the enveloping algebra of a complex simple Lie algebra, Comm. Math. Phys. 117 (1988), 581-593.

[Sc] G. W. SchwARz, "The topology of algebraic quotients" in Topological Methods in Algebraic Transformation Groups, Progr. Math. 80, Birkhäuser, Boston, 1989, 135151.

[Si] C. SIMPSON, Constructing variations of Hodge structures using Yang-Mills theory and applications to uniformization, J. Amer. Math. Soc. 1 (1988), 867-918.

[SL] R. SJAmAAR AND E. LeRman, Stratified symplectic spaces and reduction, Ann. of Math. (2) 134 (1991), 375-422.

[S1] P. SLodowy, Four Lectures on Simple Groups and Singularities, Comm. Math. Inst. Rijksuniv. Utrecht 11, Math. Inst. Rijksuniv., Utrecht, 1980.

[S2] - Simple Singularities and Simple Algebraic Groups, Lecture Notes in Math. 815, Springer-Verlag, Berlin, 1980.

Mathematical Institute, TôHoku University, Aramaki, Aoba-Ku, Sendai 980, Japan; nakajima@math.tohoku.ac.jp 